\title{
Mapping ACL to JavaMOP: \\ A Feasibility Study
}

\author{
by \\ Joshua Beltramin
}

A thesis submitted to the Faculty of Graduate and Postdoctoral

Affairs in partial fulfillment of the requirements

for the degree of

Master of Computer Science

in

Computer Science

Carleton University

Ottawa, Ontario, Canada

August, 2014

(C) 2014

Joshua Beltramin 


\section{Abstract}

Model-based testing (MBT) is a quality assurance technique where a test suite is generated from an abstract model. There are a number of different approaches to accomplish model-based testing. While state-based techniques dominate, they have a number of inherent issues. These issues have led to the pursuit of alternatives such as scenario-based approaches.

$\mathrm{ACL} / \mathrm{VF}$ is one such scenario-based approach. Developed by Dr. Corriveau and his students, the ACL/VF system provides both a language to specify an implementation-independent testable model of a specification and the tool to validate an implementation against this model. However, the current implementation of $\mathrm{ACL} / \mathrm{VF}$ has a number of issues that prevent it from being a usable solution. In particular, the current version of ACL/VF is extremely .NET3.5 specific. Unfortunately, upgrading it to a more recent version of .NET essentially amounts to a complete rewrite. Given the widespread use of Java, a most immediate research question is to determine whether or not it is feasible to reimplement ACL/VF on that platform. Our claim is that this reimplementation can be accomplished through a mapping from ACL specifications to JavaMOP monitor specifications. The following thesis provides two case studies supporting this claim as well as an element-by-element proposed mapping. 


\section{Acknowledgments}

I wish to thank my supervisor, Professor Jean-Pierre Corriveau, for his help in suggesting a topic, guiding me through it and editing this dissertation. I also want to thank my co-supervisor, Professor Wei Shi, for involving me in her Engage research project with Blueprint, as well as for her input and funding for this research.

I would also like to thank my family especially for all of their support and encouragement over the last couple of years. They have played a tremendous role in helping me achieve what I have and without them, I know would not be where I am today.

Finally, I would like to thank all of my close friends, both those I've been close to for years and those I've met while doing my Masters. Whether I needed help figuring some bug or problem out, or just needed someone to go out with to get away from the work for a while, you were always there.

Thank you all. 


\section{Table of Contents}

Abstract $\quad$ ii

Acknowledgments $\quad$ iii

Table of Contents $\quad$ iv

List of Tables viii

List of Figures viii

List of Acronyms ix

1 Introduction 1

1.1 The Context . . . . . . . . . . . . . . . . 1

1.2 Problem and Contribution .................. 4

1.3 Overview of Solution and Methodology . . . . . . . . . . . 5

2 Related Work $\quad 9$

2.1 Another Contract Language . . . . . . . . . . . . . . . . . 10

2.2 Run-Time Verification . . . . . . . . . . . . . . . . . . . . 14

2.2.1 Taxonomy ........................... 15

2.2.2 A Brief Survey of Available Technology . . . . . . . . . . . . 18

2.3 JavaMOP . . . . . . . . . . . . . . . . . . . . . . . . 24 
2.3.1 Parametric Trace Slicing . . . . . . . . . . . . . . . . . . 24

2.3 .2 Monitor Specifications _. . . . . . . . . . . . . 26

2.4 Beyond JavaMOP: JUnit . . . . . . . . . . . . . . . . . . . . . . . . . 29

3 Example Walkthroughs $\quad 30$

3.1 Overview . . . . . . . . . . . . . . . . . . . 30

3.1.1 Validation Framework Functionality . . . . . . . . . . . . . 32

3.1 .2 The Blackboard . . . . . . . . . . . . . . . . . . . . . . 32

3.1 .3 Contract Classes . . . . . . . . . . . . . . . . . 34

3.1 .4 Enhanced ACL Events . . . . . . . . . . . . . . . 36

3.2 Yahtzee Case Study . . . . . . . . . . . . . . . . . . . . . 36

3.2 .1 Significant Elements _. . . . . . . . . . . . . . 37

3.3 University Example . . . . . . . . . . . . . . . . . . . . . . . . . . 42

3.3 .1 Significant Elements . . . . . . . . . . . . . . . 43

3.3.2 Discussion of Difficulties . . . . . . . . . . . . . . . 73

3.4 Verification Technique . . . . . . . . . . . . . . 77

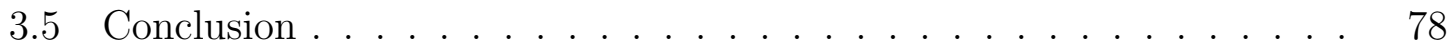

4 ACL to JavaMOP $\quad 79$

4.1 Overview . . . . . . . . . . . . . . . . . . . . . . . 79

4.2 Elements Mapping . . . . . . . . . . . . . . . . . 80

4.2 .1 Using Declarations . . . . . . . . . . . . . . . 80

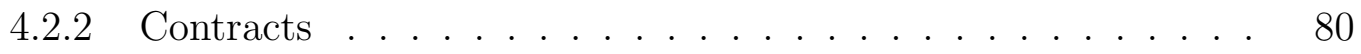

4.2.2.1 Bind Point Expression _ . . . . . . . . . . . 81

4.2.2.2 Context Access Expression . . . . . . . . . . . . 81

4.2.2.3 Value Access Expression . . . . . . . . . . . . . 81

4.2.2.4 Parameter Access Expression _. . . . . . . . . . 82

4.2.2.5 Don't Care Expression . . . . . . . . . . . . . 82 
4.2.2.6 Variables ................... . . 82

4.2.2.7 Parameters .................. . . 83

4.2.2.8 Structure ................. . . 83

4.2.2.9 Observability Methods . . . . . . . . . . . 83

4.2.2.10 Invariants . . . . . . . . . . . . . . 84

4.2.2.11 Responsibilities . . . . . . . . . . . . . 84

4.2.2.12 Responsibility Bodies . . . . . . . . . . 85

4.2.2.13 Stub Responsibilities . . . . . . . . . . . . 87

4.2.2.14 Scenarios . . . . . . . . . . . . . . . 87

4.3 Summary of Mappings . . . . . . . . . . . . . . . . . 91

5 Conclusion and Future Work $\quad 96$

5.1 Summary of Contribution . . . . . . . . . . . . . 96

5.2 Future Work . . . . . . . . . . . . . . . . . . 97

5.2.1 Custom ACL Scenario Formalism . . . . . . . . . . . . . . 98

5.2.2 JavaMOP Abstract Monitors . . . . . . . . . . . . . . . . . 98

5.2 .3 JavaMOP Inheritance . . . . . . . . . . . . . . . . . 98

5.3 Conclusion . . . . . . . . . . . . . . . . . . . . . . . . . . . . 99

$\begin{array}{lr}\text { References } & 100\end{array}$

Appendix A Contract Class Example 106

A.1 Course Contract Class . . . . . . . . . . . . . . . . 106

Appendix B Log Examples 109

B.1 Course Checks Log . . . . . . . . . . . . . . . 110

B.2 Course Scenario ReportMarks Log . . . . . . . . . . . . . . . . . . . 110

B.3 Student Checks Log . . . . . . . . . . . . . . . . . . . 111

B.4 Student Scenario RegisterForCourses Log . . . . . . . . . . . . . . . . 114 
B.5 Student Scenario TakeCourses $\log \ldots \ldots \ldots \ldots$

B.6 University Checks Log . . . . . . . . . . . . . . . . . . . . 121

B.7 University Scenario CreateCourses Log . . . . . . . . . . . . 122

B.8 University Scenario CreateStudents Log . . . . . . . . . . . . 123

B.9 University Scenario Term $\log$. . . . . . . . . . . . . . . . . 123

B.10 Test Driver Program . . . . . . . . . . . . . . . . . 124 


\section{List of Figures}

1 Taxonomy of runtime verification $[26] \ldots \ldots \ldots \ldots$

2 Relationships between system elements. . . . . . . . . . . . . 34 


\section{List of Acronyms}

\begin{tabular}{ll}
\hline Acronyms & Definition \\
\hline \hline ACL & Another Contract Language \\
CFG & Context-Free Grammar \\
DbC & Design by Contract \\
ERE & Extended Regular Expression \\
IEEE & Institute of Electrical and Electronics Engineers \\
IUT & Implementation Under Test \\
LTL & Linear Temporal Logic \\
MaC & Monitor and Checking \\
MBT & Model-Based Testing \\
MEDL & Meta-Event Definition Language \\
MOP & Monitor Oriented Programming \\
PEDL & Primitive Event Definition Language \\
QA & Quality Assurance
\end{tabular}


RV Runtime Verification

TRM Testable Requirements Model

VF Validation Framework 


\section{Chapter 1}

\section{Introduction}

The following chapter will define the problem we address and the solution we propose. To begin, we present a brief overview of the problem and its context. Namely, the importance of testing specifications on an implementation under test (IUT) and how $\mathrm{ACL} / \mathrm{VF}$ provides a different approach from the work that currently dominates the field of model-based testing. Following this will be a description of our contribution and a more detailed explanation as to why it is necessary. The chapter will then conclude with a summary of our solution and the methodology we used to verify that it does indeed provide a solution to the problem.

\subsection{The Context}

Software quality assurance (hereafter QA) is a vital process in any software development lifecycle. It defines how the individual or team ensures that the project under development not only meets the customers needs but does so with minimal faults [1]. More broadly speaking, software QA ensures that a software system meets the purpose for which it was intended.

A primary process within software QA is testing. One purpose of software testing is to ensure that the system being developed conforms to the requirements laid out 
in the specification document [2]. This is accomplished through either the execution or static analysis of the IUT and generally consumes 40 - 50 percent of development efforts and 40 - 50 percent of development budgets [2,3]. As important as testing is, software is often not tested as thoroughly as it should be [4].

Testing as a process can be viewed as consisting of four hierarchical levels of abstraction. These levels are, starting from the lowest level, unit testing, integration testing, system testing, and acceptance testing [5]. At each level, a different aspect of the system under development is tested.

Unit testing is a form of white-box testing where the developer verifies that each individual component of the project functions properly as a unit [6]. With respect to the object oriented paradigm, classes and the individual methods within them are tested in isolation to ensure proper functionality, without considering interactions with other classes [6]. A common framework used to accomplish this level of testing is the XUnit framework, an example of which is JUnit [7], a common unit testing framework used for projects written in the Java programming language.

At the next level of the hierarchy is integration testing. After testing components at the unit level, they are integrated together, or assembled, into subsystems and finally into systems. Despite unit testing, it is possible, if not frequent, that components fail when integrated together [5]. The purpose of integration testing is therefore to verify that the interfaces between modules, or collections of classes, are correct and that they interact properly.

Following integration, a set of high-order tests are executed to verify that the requirements established during the requirements elicitation phase have been satisfied and that the system as a whole functions properly. This stage is known as system testing. A form of black-box testing, it ensures that the developed software meets all of the functional and non-functional requirements [5]. More generally speaking, system level testing validates the system against its intended specification [8]. 
Lastly in the hierarchy is acceptance testing. Acceptance tests represent the customer's interests. That is, they are tests that give the customer confidence that the application that has been developed has the required features and that these features behave correctly [9]. These tests are either specified or applied directly by the customer or the customer's representative [1].

Orthogonal to these levels of testing, testing techniques can be categorized as either code- or model-centric [4].

Code-centric testing, such as Test-Driven Design (TDD), uses test cases written at the implementation level in order to guide development of the software [10]. These test cases do not rely on any form of requirements model and as a result provide no traceability to such a requirements model [4]. As well, the test cases are code artifacts and as such are implementation-driven and implementation-specific [4]. This significantly reduces the reusability of the test cases.

Model-centric approaches, such as Model-Based Testing (MBT) on the other hand generates a test suite from an abstract model. This model is built using the specifications of the stakeholders and in general captures a partial, implementationindependent representation of the IUTs behaviour due to its abstract nature [10]. The use of such an implementation-independent model provides two significant advantages: 1) A decoupling of requirements from the IUT and 2) The reuse of the test suite across several IUTs. [4]

Of the various model-based approaches to system level testing, those built upon state-based specifications dominate $[4,11]$. These methods however have limitations that need to be overcome if a software testing tool is to scale up to industrial usage $[4,12]$. These limitations will be discussed further in Chapter Two.

For now, we will simply remark that Grieskamp [12] states that scenario-based specifications are generally preferred by stakeholders to state-based specifications. An alternative to the state-based approach is the MBT tool called Another Contract 
Language (hereafter ACL) and its corresponding Validation Framework (hereafter $\mathrm{VF}$ ). The premise of ACL/VF is to provide a software validation framework built on two already well-known and established concepts. These are: a) the expression of the requirements in terms of responsibilities and scenarios, and b) organizing these responsibilities and scenarios into contracts [10]. As well, ACL/VF adopts the premise of a genuine model-based outlook where the specification must be implementation

independent [10]. To accomplish system level testing, the ACL/VF tool executes the ACL contracts alongside an IUT, as explained in Chapter Three, with information being exchanged between these two executions in order to support verification of the specification. While not strictly a runtime verification tool due to its incorporation of static checking functionality, the majority of ACL/VF's quality assurance tasks occur at runtime. In particular, scenario monitoring forms the core concept behind the ACL/VF tool.

\subsection{Problem and Contribution}

While ACL/VF provides a solution for system level testing, a number of issues remain with it that are inherent to its design. The first of these is a major bug that has been revealed after extensive experimentation with ACL/VF. This consists in an inability of ACL/VF to distinguish properly between multiple instances of a single class executing concurrently during scenario monitoring (explained in Chapter Two). Another issue is that some of the tools and mechanisms used within ACL/VF are complex and extremely .NET 3.5 specific [13]. This complexity and dependency upon .NET 3.5 limits any future work on the current VF to handle newer versions of the .NET framework or any other implementation languages independent of the Microsoft Intermediate Language. In particular, ACL/VF relies heavily on: a) Microsoft Phoenix to capture the required IUT binding information (explained in Chapter Three) as well 
as static checks, and b) programmable breakpoints to pause IUT execution for checking against the testable requirements model (hereafter TRM) [13] captured in ACL. This critical functionality of the tool would need to be either dropped or completely redesigned to be able to adequately update ACL/VF.

To determine the feasibility of redesigning and reimplementing ACL/VF, Dr. Corriveau's research group has extensively examined its source code. We have come to the conclusion that the optimal solution is to not attempt a port to a newer version of the .NET platform. In place of a port, we have decided to instead potentially improve the applicability of ACL/VF by having it redeveloped for the Java environment. Java was chosen due to its widespread use over similar languages such as $\mathrm{C}++$ or C\#. Thus, the key questions to address before proceeding with any attempt to reimplement ACL/VF is: is it feasible to support ACL syntax and semantics, especially its use of scenarios, in some technology that is Java-based. The contribution of this thesis is to provide an affirmative answer to this question.

\subsection{Overview of Solution and Methodology}

Another contract language (ACL) [13] is a high-level specification language created by Arnold et al. [10] to support a strongly-typed, implementation-independent testable requirements model for several candidate IUTs. ACL is non-diagrammatic and is constructed based on the concept of scenario contracts as proposed by Nebut et al. [14].

One of the core concepts of ACL is the contract. Contracts in ACL are specifications of IUT types and are bound to these types for both conducting static checks of the bound types as well as runtime verification. This binding to types occurs prior to runtime and consists in associating each contract to an IUT type. At runtime, instances of contracts are created (a concept explained shortly) and bound to their 
corresponding type instances. The pairs then execute simultaneously, exchanging pertinent information for the runtime verification (as explained in Chapter Three).

Contracts in turn are composed of a number of key elements. The first of these are responsibilities. A critical element of ACL, responsibilities are the functional requirements or tasks of a contract. Another key element is the contract variable. These are variables that exist within the context of the contract and have the same lifespan as the contract instance to which they belong. ACL events are also important. An event is fired by a responsibility or a scenario, and is global to all currently executing contracts instances as well as those that are triggered by this event. Events act as a communication mechanism between contracts. Finally, a scenario defines an ordering of events and responsibilities for the VF to observe at run-time, as will be illustrated later. In other words, scenarios are what are used to drive the runtime verification of the ACL/VF tool.

There are a number of significant aspects of contracts and scenarios in ACL that need to be addressed. These will be briefly overviewed here and discussed in detail in Chapter Two. The first aspect is that a contract may have several instances existing simultaneously, each with its own set of instance-bound contract variables as mentioned above. As such, the tool must be able to distinguish between these instances at runtime. That is, upon occurrence of a responsibility-bound method, the tool must be able to properly identify the corresponding contract instance to which the responsibility belongs. For example (as will be illustrated in the University Example, Section 3.3), a university will have multiple students attending it simultaneously.

Following a contract's ability to have more than one concurrent instance, a contract may also contain multiple scenarios where each scenario may have one or more instances in execution simultaneously. For example, a student (one of possibly many) may take multiple courses concurrently (i.e., that student has multiple instances of the TakeCourses scenario) and while that student is taking courses, a number of other 
students may be registering or taking courses (RegisterCourse and TakeCourse scenarios). In other words, there are multiple instances of contract Student and each such instance may have several instances of some of its scenarios. ACL contracts and, more specifically, the scenarios contained within them can become quite complex at runtime. The proper handling of the various instances that may exist simultaneously is not a trivial problem and requires serious consideration.

Given these complexities of ACL's semantics and the operational demands they impose on a run-time verification system, our overall goal is two-fold. First, we must determine which of the available technologies for runtime verification can support ACL, and in particular, its runtime scenario monitoring. Second, we must demonstrate that the selected technology indeed adequately supports ACL. To do so, we will tackle Arnold's [15] complex university example.

The solution we have come up with is a combination of two different technologies: the JavaMOP framework [16] and JUnit [7]. Specifically, JavaMOP 3.0. This pair of tools enables us to properly handle the various elements and intricacies of ACL specifications. We use the JavaMOP framework to generate AspectJ monitors for parametric runtime monitoring (discussed later) of a Java IUT with JUnit assertions to execute the test oracles checks throughout the execution of an IUT.

The ultimate goal for the complete reimplementation of ACL/VF is to be able to do so while maintaining as much of the original ACL syntax and semantics as possible. This thesis is the first of several for this research project. Our goal was to demonstrate the feasibility of scenario modeling before any more time was invested towards this reimplementation. We will show in this thesis that a combination of JavaMOP with JUnit assertions can handle the complex scenario monitoring of ACL/VF.

In addition to this thesis, a number of separate theses are being conducted. The first will be taking the results of this thesis and using them to develop the reimplementation of ACL/VF. This will be done on top of Java through the use of JavaMOP 
with AspectJ and JUnit. Another student will also be working on adding to the ACL syntax and generating the necessary bindings between ACL/VF and the IUT.

New syntax will be coming from two other theses. One will be using ACL with new generative features to model some of the Group of Four design patterns [17]. The second will be developing an ACL model to illustrate how interleaving and loops can be handled without leading to a combinatorial explosion of tests generally reported in the literature [12]. This work will be based on a well known system-level case study [8].

The rest of this thesis will be structured as follows. Chapter Two will review the various technologies relevant to our work with an emphasis on run-time monitoring tools. The goal of that chapter is to motivate our choice of technology, namely JavaMOP. Chapter Three will then present several examples we developed with the intent of conveying the modeling strategies we developed for scenario monitoring using JavaMOP. The goal of this chapter is to explain how we came up with the strategies we developed for our mapping of ACL to JavaMOP. Chapter Four will then summarize our work and the examples discussed in Chapter Three but outside of the context of those specific examples. The mappings discussed in Chapter Four are now at the basis of the ongoing efforts to generate JavaMOP out of ACL. Lastly, Chapter Five will discuss our conclusions and future work. 


\section{Chapter 2}

\section{Related Work}

As was mentioned in Chapter One, state-based MBT tools have a number of inherent issues. These are described in [12] and consist of problems such as state explosion ${ }^{1}$, test selection, and test management.

The most prominent of these issues is state explosion. Traditional state-based tools take the approach where a state is a set of values (the instance variables, or equivalently data members of) held by objects of a system. In this type of traditional state-based system, combinatorial explosion comes from the number of variables as well as the possible values for each variable [18]. More recent work in the field of MBT relies on approaches where the states are not rooted in the variables of objects but instead each state may pertain to a sequence of method calls $[2,12]$. This can be seen in tools such as Spec Explorer [12] and temporal logic approaches [19-21] where the temporal logic formula are translated into state machines. More specifically, in Spec Explorer, the state explosion comes from both the possible sequences of method calls as well as the valid sets of parameters for those calls. This is also the case with temporal logic approaches; at least for those that parameterize the logic. That is, approaches that do not include parameters have no way to verify whether a valid or

\footnotetext{
${ }^{1}$ The problem of state explosion may be somewhat remedied with the use of extended FSMs. That is, FSMs with state variables.
} 
invalid method call has occurred as parameter values would need to be checked.

\subsection{Another Contract Language}

ACL is a high-level, implementation-independent specification language, the goal of which was to provide a new approach to a testable requirements model (TRM) $[10,22,23]$. It is a textual specification and is intended to be implemented after the necessary user requirements notation (URN) specifications have been developed. More specifically, ACL is intended to be written based on refinements of the responsibilities, scenarios, and metrics of goal-oriented requirements language (GRL) and use case map (UCM) diagrams (developed from use cases) [22].

The following section will provide a more detailed example of the significant elements of ACL contracts as given in [10]. Following each element, an example from [10] for an ACL container project will be used to illustrate how it is implemented in ACL.

Bound to an IUT type, an ACL contract defines the functional requirements of a type as well as the various scenarios in which the functional requirements may occur. More specifically, the scenarios contained within an ACL contract are grammars of responsibilities where each responsibility corresponds to a functional requirement of the IUT. These responsibilities can be bound to either procedures from within the IUT or they can be decomposed into sub-grammars of responsibilities. There are also two special types of responsibilities in ACL called new and finilize. These correspond to class constructors and destructors. ACL also includes some elements derived from the Design-by-Contract paradigm [22]. These are pre- and post-conditions, and invariants. Pre- and post-conditions are applied to responsibilities and are used to check the state of the IUT before and after the execution of the bound procedure. Invariants are also used to check specific conditions, but are checked both before and after execution of every procedure to which a responsibility is bound. 
The following is an example of a bound responsibility. The responsibility is bound to a method that takes an item as a parameter and has no return type. It also contains the pre- and post-conditions to make sure the item is added properly. The Execute() statement is used to control when the bound method may execute.

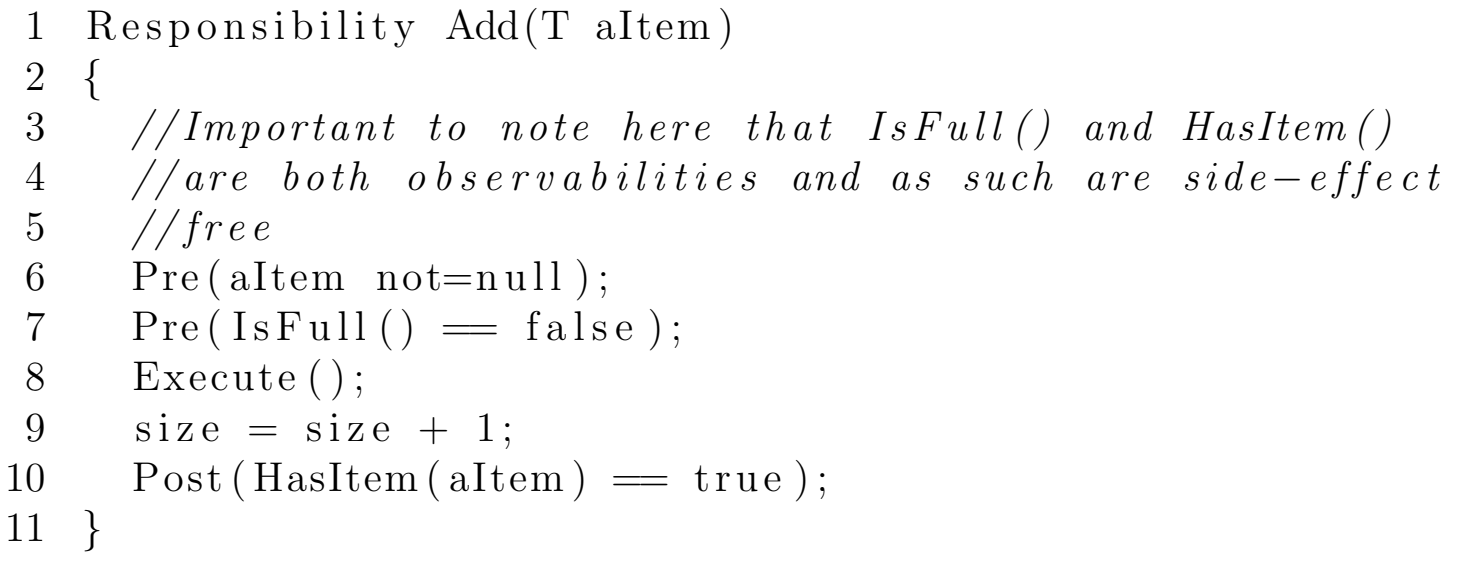

Contracts may also contain local variables (e.g. the size variable in the example below). As was discussed previously, contract-scope variables are dynamic variables that have the same lifespan as the contract instance to which they belong. They can be used to keep track of data, independent of the IUT. As such, they can be used as dynamic test oracles over the lifespan of the contract. ${ }^{2}$ This is shown in the following example. This example will also illustrate an invariant definition in ACL. The invariant checks that the container contract instance at hand always has zero or more items and that the number of items the contract has recorded matches the number of items the IUT class has recorded.

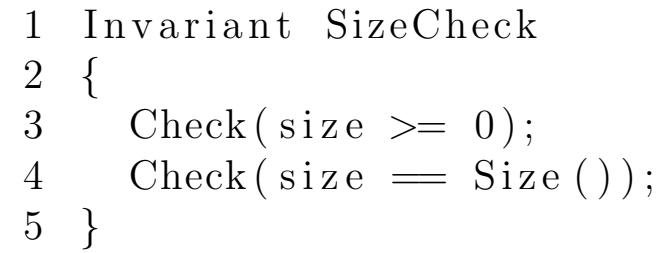

\footnotetext{
${ }^{2}$ This feature is not unique to ACL and may also be found in specifications such as JML [24]. JML does not however support scenario as can be found in ACL.
} 
Observabilities are another important element of ACL. They are what are used to retrieve state information from the IUT. To do so, they can be either bound, where they have a corresponding IUT method they use to retrieve the information, or they can be unbound, where they are used to compute a value based on the value of some contract variables. The following example shows a number of observability definitions including the Size() observability seen in the invariant above. As can be seen, observabilities support a number of different return types and can take parameters that can be used to query the IUT.

1 Observability Boolean IsFull ();

2 Observability Boolean IsEmpty ();

3 Observability $\mathrm{T}$ ItemAt(Integer index);

4 Observability Integer Size ();

ACL also supports events. Events in ACL are occurrences that may be relevant outside of the context of the contract in which they occur. All contract instances are notified of event occurrences whenever an event is fired with the fire statement. Only those contracts that contain an observe statement for the fired event will take any action when the event is fired. This is illustrated in the following example of an event dependent scenario. The scenario is initialized by the Trigger keyword when an item is added to a container, waits for the search responsibility to occur and is then terminated upon observation of the ItemRemoved event. In this example a responsibility occurrence is used to trigger the scenario while an event occurrence is used to terminate. This is not mandatory, but simply used to illustrate how an event may be used. Also worth noting here is another syntactic issue with ACL. The ItemRemoved event is specific to a single item being removed. However, there is currently no way in ACL for the active scenario instances to identify which item has been removed. Therefore, if there is more than one active instance waiting for the ItemRemoved event, all would terminate instead of only the instance corresponding 
to the actual item that has been removed.

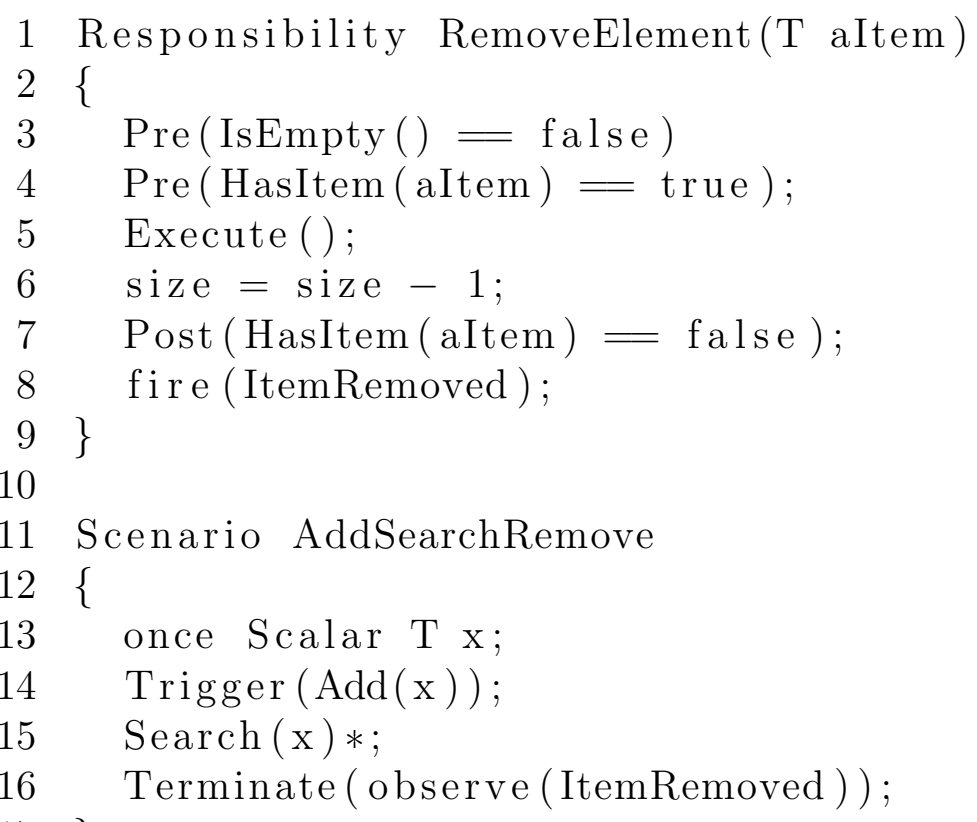

The primary element of ACL contracts, scenarios define the temporal aspects of the IUT. They begin with the trigger statement that contains the condition required for an instance of the scenario to be initialized. Upon initialization, the scenario instance monitors for expected responsibilities or events based on the contained grammar. The terminate statement denotes the condition required for the scenario to finish successfully. Scenarios may also contain their own local variables for use within the scenario.

The following example illustrates a very basic scenario that enforces the requirement that an item (defined as the variable $\mathrm{x}$ ) must be added to a container before it can be removed from it. It is a slight modification of the previous AddSearchRemove scenario to show a proper implementation without the indeterminate nature of the termination event. The scenario is initialized upon the occurrence of the $\operatorname{Add}()$ responsibility and terminates upon the occurrence of the RemoveElement() responsibility, as long as the element being removed is the same as the one that was added. 
The use of the variable $\mathrm{x}$ for an item parameterizes the scenario so that for successful completion, the same element that was added must to be removed.

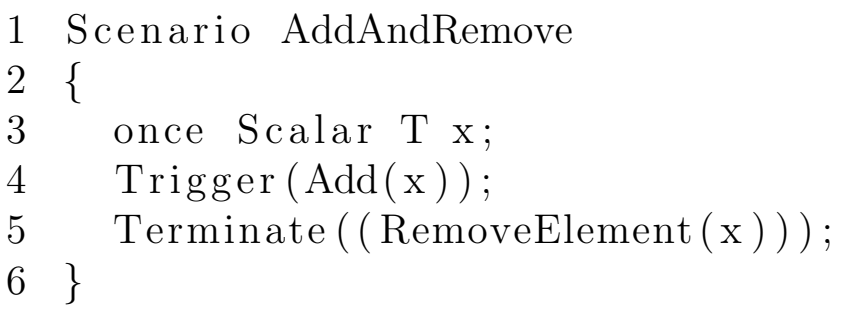

See [10] for the complete code and descriptive annotations from which the above examples were taken.

It is also important to note that, for the purposes of this thesis, we support a newer version of ACL. This newer version includes parameterized events in fire and observe among other minor modifications to the language. The implementation of this new version of ACL is currently under investigation as a separate thesis.

\subsection{Run-Time Verification}

As defined by the IEEE [25], verification is the process by which a software system is evaluated with the purpose of providing evidence that it conforms to the requirements. Traditionally, this software verification process comprised methods such as theorem proving, model checking, and testing. More recently, runtime verification has emerged as a relatively new approach to software verification [26].

Runtime verification is the application of verification techniques that allow for the checking of whether or not a run of an implementation under test satisfies or violates given correctness properties [26]. This is generally achieved through the synthesis of monitors from some high-level specification.

In terms of monitoring an IUT, a run is understood to be a possibly infinite sequence of system states formed by current variable assignments, or as the sequence of 


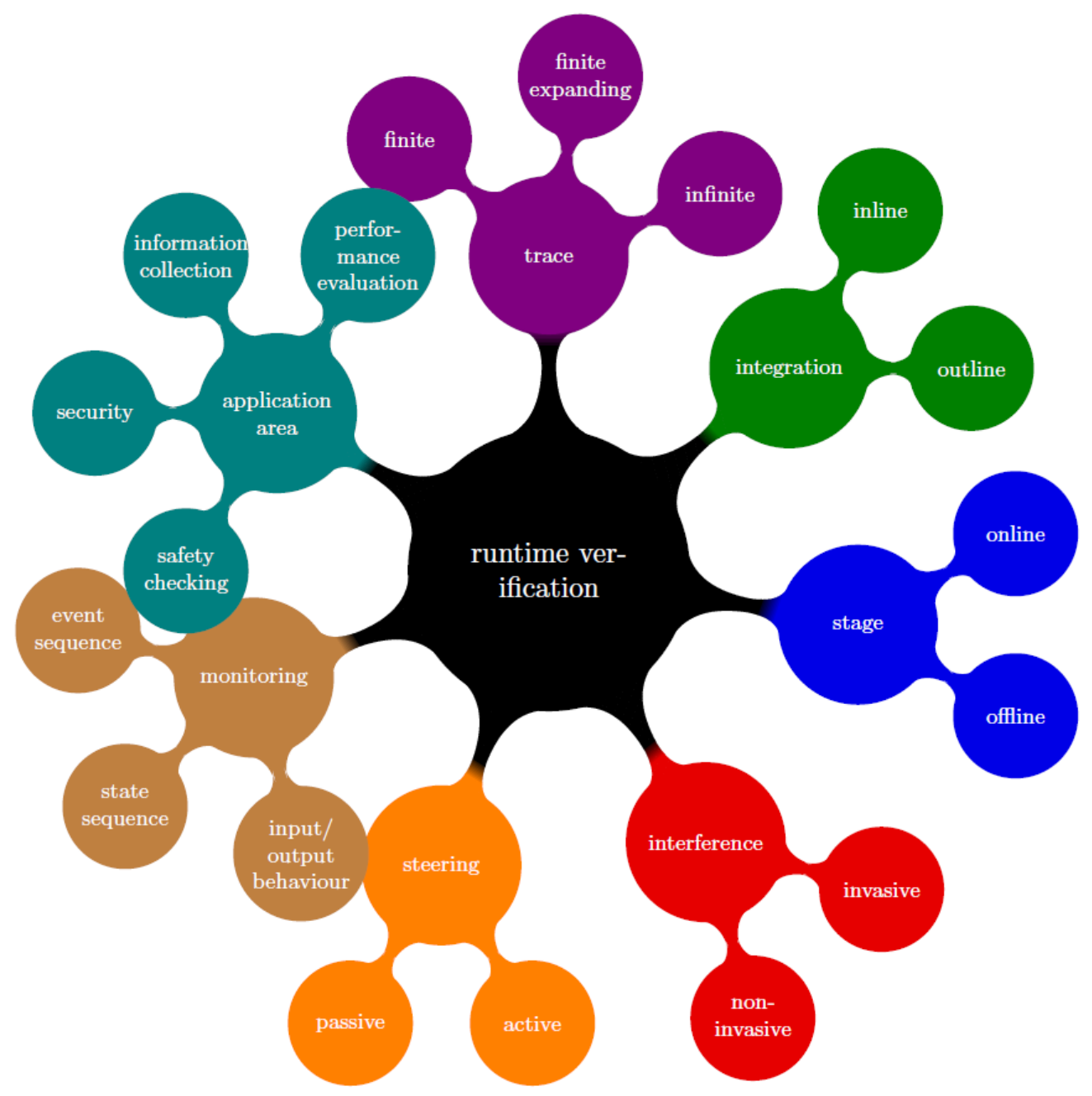

Figure 1: Taxonomy of runtime verification [26]

input/output actions of the IUT [26]. An execution on the other hand is a finite prefix of a run, or more formally, a finite trace [26]. With respect to runtime verification, we are concerned with reading an execution of an IUT and generating a certain verdict based on this reading. This is the function of a single or a set of runtime monitors.

\subsubsection{Taxonomy}

With the growing interest in runtime verification, a variety of approaches to accomplishing it have emerged $[26,27]$. The following section will give a brief overview of the 
taxonomy provided by Leucker [26] as seen in Figure 1 before discussing the current work in the field.

A primary concern and category for classification of runtime verification is the desired application area. This will control how the monitor is designed and what its purpose will be. Of the surveyed technologies, four application areas were derived. The most commonly observed was safety checking [26], followed by security conditions. Runtime verification may also be used however to collect information on the IUTs execution or to provide a performance evaluation.

Runtime verification solutions may also use one of three parts of a run for the monitoring: the IUTs input and output behaviour, one of its state sequences, or a sequence of events derived from the IUTs execution.

The next category in the taxonomy is trace. Trace refers to the type of trace the runtime verification monitor uses to track the execution of the IUT. Regardless of the method used, monitoring of a trace will ultimately result in some form of true or false verdict for the property being monitored.

One must also consider how to monitor the IUT. That is, how will the monitor actually know or gather the necessary information to know what is happening or has happened in the IUT during execution. This is known as integration and can either be inline, where code may be interweaved with the IUT being checked, or outline, where the monitoring code may be used to synthesize a monitoring device. Also considered when determining how to monitor the IUT is the stage. The stage refers to when the verification occurs. Online staging refers to when the monitor is checking the execution at runtime, necessary for inline integration, where offline staging refers to checking a finite set of recorded executions.

A monitor may also directly influence the IUT during execution in one of two ways. The first of these is monitor interference. Interference refers to whether or not a monitor impacts the execution of the IUT by running on the same hardware. If on 
the same hardware, a monitor is said to be invasive, where if using separate hardware to monitor, it is said to be non-invasive. The second means of influence is whether or not the monitor steers the IUT. That is, when a failure is detected, does the monitor simply record the failure, or does it take actions to steer or heal the IUTs execution. The monitor is therefore labeled as passive or active respectively.

In addition to the above taxonomy, we also add two additional categories for our work. These are the target implementation language and the specification type.

Since our goal is to provide a tool for the Java environment, the target language categories are Java and Other. A tool may of course fall within both of these categories as can be seen in Table 2.1 .

The categories for specification type are Assertion Based, Temporal Assertion Based, State Based, and Scenario Based. Assertion-based specifications are those that only check conditions on the IUT. That is, specifications that only specify conditions such as pre- and post-conditions and invariants. Temporal Assertion Based specifications are those that define expected system operation in terms of properties specified using some temporal formalism such as a temporal logic or context-free grammar $(\mathrm{CFG}){ }^{3}$ State-based specifications are those that specify changes in the state of the IUT. More specifically, they focus on a sequence of states where a state is a set of values of some variables at some point in time. Lastly, scenario-based specifications are those that specify sequences of events within the IUT where an event is an occurrence such as a method invocation.

\footnotetext{
${ }^{3}$ These can ultimately be equivalent to state-based methods. However, the support for the concept of a grammar such as a CFG as opposed to states makes them a better solution for scenario monitoring as is in ACL. In other words, the conceptual traceability between ACL scenarios and a CFG formalism is much more desirable than trying to map ACL scenarios into explicitly stateoriented formalisms. As well, it is typically believed that CFGs are more expressive than state machines.
} 


\subsubsection{A Brief Survey of Available Technology}

With the above taxonomy in mind, we will now provide a brief survey of the work that has been done in the field of runtime verification. This survey will cover both old and new tools and categorize them within a specific set of criteria. The criteria used for the selection of an appropriate tool were as follows: 1) The tool must claim an actual implementation, not just present a theoretical solution. As this thesis is dependent on an actual implementation, theoretical solutions ( [28-30]) are excluded from further categorization of tools. 2) The tool must use online staging and be implemented for the Java environment. This is necessary to be able to handle the same type of runtime verification as the VF where contracts are executed against an IUT (see Chapter Three). 3) The tool must not be solely state-based for the reasons mentioned previously, or solely assertion-based. In other words, the tool must provide a means of specifying an IUT in terms of scenarios, or one able to handle a grammar of events, (needed to be able to properly handle the scenarios of ACL). 4) Lastly, beyond scenario or grammar monitoring, the tool must be able to handle the elements of the design-by-contract paradigm included in the ACL semantics.

All considered tools are listed in Table 2.1. The columns correspond to the categories of the taxonomy discussed above that were considered relevant to the needs of this thesis.

These tools were found through a systematic literature review. First, a search through prominent journal databases (IEEE, ACM, SpringerLink) for surveys of runtime verification tools was used to compile a list candidates. The papers on the tools from these surveys were then collected and reviewed to both categorize the tools as well as identify further candidates. As well, additional keyword searching ${ }^{4}$ through the aforementioned databases was used to further identify candidate tools. A total

\footnotetext{
${ }^{4}$ Keywords such as "runtime verification", "runtime monitoring", "Design-by-contract", "system testing", etc. were used.
} 
of 30 tools and their respective papers were found. Also important to note is that some possible candidate tools such as JML were not included as other tools we did include, such as RAC, were built on top of and include the functionality of these omitted tools. While thorough, there are likely still candidate tools that were missed in the review. However, we are confident that this review was more than adequate to find some, if not all, of the best candidates for our purpose.

After analysis of the tools in Table 2.1 the following eliminations were determined. By criterion two, tools [31-42] were not considered as potential tools. Criterion three removed tools [43-51] from consideration as potential solutions.

The following tools are the only ones that satisfied criterion three and were left for further consideration. Tracematches [52] and JLO [19] are both based on the aspectoriented paradigm and use regular expressions and LTL respectively. As well, they support parametric events, i.e., free variables can be used in event patterns and are bound to specific values at runtime for matching events. Java PathExplorer (JPaX), JEagle, and TemporalRover use as specifications LTL, fixed-point logic, and future time metric temporal logic respectively. JPaX and JEagle use automatic byte-code instrumentation to extract the necessary events for monitoring while TemporalRover generates executable code as assertions written as comments in the IUT source code. All three tools, regardless of how events are extracted, monitor the resulting event stream and check it against their respective specifications. RuleR was built to address efficiency weaknesses identified in the Eagle RV framework [53]. A low-level rule system, RuleR acts as a kind of "byte-code" for runtime monitoring that other higher level specifications such as LTL could theoretically be translated into [53]. Ultimately, RuleR was designed to provide a simpler, more efficient alternative to Eagle-type tools. The SPIN RV tool specifies system safety properties using LTL, much like previously discussed approaches. It uses these LTL properties though to generate Büchi automata for the runtime verification. The key difference between SPIN and 
most other RV tools however is that SPIN correctness properties are used to formalize potential violations of correct system behaviour. Lastly on the list is LoLa. LoLa is a synchronous specification language. It allows the user to specify properties in past and future LTL. The important aspect to note about all of these approaches is that while they could all handle ACL's scenario semantics, none of them can handle the additional assertions, as stated in criterion four, necessary to fully map ACL to the desired tool or framework.

Left as potential candidates were Java-MaC and JavaMOP. JavaMOP remained viable because while not specifically supporting assertions such as pre- and postconditions, it supports user defined event actions. In other words, custom Java code can be written for each event. This provided a means of including the types of assertions necessary through the use of an additional tool such as jUnit (discussed later).

Java-MaC is a runtime verification tool designed on top of the Monitor and Checking $(\mathrm{MaC})$ architecture. It provides automatic source code instrumentation and runtime checking based on an interval temporal logic based specification. As well, Java-MaC provides separation between monitoring low-level, program-dependent behaviour and checking high-level behavioural requirements [54]. This separation keeps the high-level specification independent of the implementation, enabling reuse for either changes in the IUT or across different IUTs.

The specification of Java-MaC consists of two parts. The first handles low-level specifications and is called the Primitive Event Definition Language (PEDL). This specification is implementation-dependent and is used to define what from the IUT is an event. The events from PEDL are what are passed on to the high-level specification for verification. This high-level specification is called the Meta Event Definition Language (MEDL). It uses temporal logic to define correctness properties of the IUT. As well, MEDL supports auxiliary variables whose values can be used to define 
events and conditions. These variables however are quite primitive in that they can only be numeric and used to check number of occurrences of events.

The second candidate tool, JavaMOP is built on the concept of keeping track of and matching a trace at runtime against a formally defined property. This property can be defined in a number of different logical formalisms such as linear temporal logic, extended regular expressions, or context-free grammars. JavaMOP also supports parametric monitoring through parametric trace slicing (described in detail later).

Monitoring parametric properties allows an individual to describe not only global behaviours of an IUT, but also behaviours of the objects within the IUT [16]. As such, it provides a more thorough solution than Java-MaC for runtime verification. While many tools have provided parametric monitoring solutions [16], JavaMOP's ability to define properties using a variety of formalisms provides a stronger solution. This is due to the fact that some properties can be monitored more efficiently using different formalisms [16].

As well, JavaMOP includes event actions associated with each event. An event action is a block of standard Java code that is executed when the event is reached during execution of the IUT. Event actions provide a way for developers to include any functionality desired as long as it is valid Java code. For example, logging functionality could be included, or assertion checks with JUnit (discussed later).

While both Java-MaC and JavaMOP provided viable solutions as RV tools that could be mapped to from ACL, there were a number of issues that made JavaMOP the preferred tool.

One issue is the inherent limited expressiveness of both regular expressions and temporal logic due to their reduction to automata (mentioned previously) when monitored [55]. JavaMOP overcomes this issue by being implemented as logic-independent. That is, JavaMOP supports a number of different types of logic formalisms and allows users to implement their own if so desired. Specifically, JavaMOP includes support for 
context-free grammars (CFGs), which avoid any type of reduction to automata [55] and map relatively nicely to the concept of a scenario.

Another issue is the limited capabilities of tools such as Java-MaC. While Java$\mathrm{MaC}$ does support auxiliary variables, they are limited in functionality. Other tools, mentioned above, do not even provide any form of auxiliary variables and can only be used to check the temporal properties.

Ultimately, JavaMOPs expressiveness and extensibility in terms of specification properties, its ease-of-use when compared to similar tools (based on our own experimentation), and its scalability [16] made it the best choice for our purposes. As well, due to the extensibility of JavaMOP in its logical formalisms, all approaches listed and discussed above could fall within the general MOP architecture, provided the appropriate logic-plugins are defined [56]. 


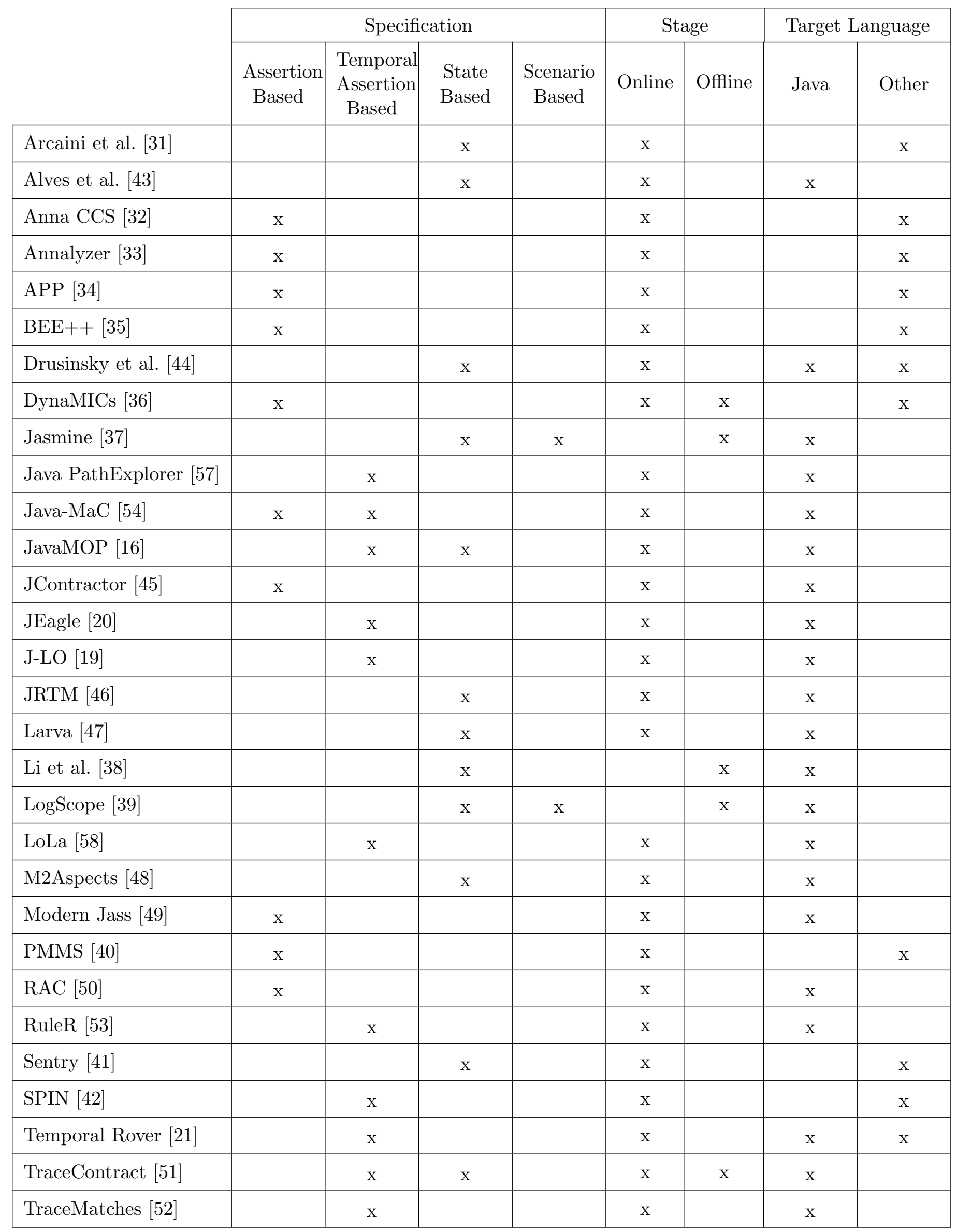

Table 1: Categorized related works. 


\section{$2.3 \quad$ JavaMOP}

An instance of the generic MOP framework [56], JavaMOP provides a means to specify concise descriptions of parametric properties through the use of a combination of event specifications (an extension of AspectJ) and properties specified over these events [16]. The specifications are used to generate AspectJ code for monitoring that is then weaved into a target program by any AspectJ compiler. As a result, the generated monitoring code observes the program's execution, records the events defined in the specification, and checks whether the execution is compliant with that specification [16].

JavaMOP also provides the additional functionality of being able to supply userdefined actions, called handlers, to execute upon either a match or failure of the specified property, or upon recognition of the occurrence of a specified event. In this way, JavaMOP provides an extremely large amount of freedom in the functionality of its monitors. This will all been illustrated in Chapter Three.

One of the most significant features of JavaMOP that makes it one of the best runtime monitoring systems [16] is its support of efficient formalism-independent parametric monitoring. In place of using hardwired formalisms, as in many of the tools outlined in Chapter Two, JavaMOP supports formalisms in the form of plugins. The user specifies which plugin they wish to use in each monitor specification. This use of plugins also allows for users to define their own plugin, resulting in a very extensible framework.

\subsubsection{Parametric Trace Slicing}

Parametric trace slicing is the mechanism by which JavaMOP monitors parametric properties. That is, properties specific to a set of parameter instances. It is one of the core concepts of JavaMOP and, as such, it will be described in detail in the following 
section. Formal definitions of the concepts discussed below can be found in [59].

A trace in JavaMOP is any finite sequence of events. That is, as events are observed and recorded by a monitor, they form a finite sequence, defining the current execution of the IUT. This trace is what is then used to check against the specification's defined property.

Given the above definition, a parametric trace is therefore a parameterized sequence of events. That is, each event in the trace has a corresponding set of parameters associated with it. At runtime, these parameters have concrete objects bound, or mapped, to them. This mapping of concrete objects to parameters is called a parameter instance.

For example, if $X=\{\mathrm{s}, \mathrm{c}\}$ is a set of parameters (of types \{Student, Course respectively), and $V=\left\{s_{1}, c_{1}\right\}$ (where $s_{1}$ and $c_{1}$ are instances of the Student and Course types), then ( $\left.\mathrm{s} \mapsto s_{1}, \mathrm{c} \mapsto c_{1}\right)$ is a parameter instance, AddStudent( $\mathrm{s} \mapsto s_{1}, \mathrm{c}$ $\left.\mapsto c_{1}\right)$ and RemoveStudent $\left(\mathrm{s} \mapsto s_{1}, \mathrm{c} \mapsto c_{1}\right)$ are parametric events, and the sequence AddStudent( $\left.\mathrm{s} \mapsto s_{1}, \mathrm{c} \mapsto c_{1}\right)$, RemoveStudent $\left(\mathrm{s} \mapsto s_{1}, \mathrm{c} \mapsto c_{1}\right)$ is a parametric trace.

Following from the above, properties in JavaMOP are functions, defined using one of the available logic plugins, that partition the set of traces into categories [56]. These categories are dependent on the formalism being used but could include categories such as violating traces, validating traces, or undecided traces. It is important to note that the actual properties defined in the monitors are independent of any parameter instances. That is, each monitor instance has an associated parameter instance and property (or properties), and it is the monitor's responsibility to determine if the parameterized events (forming the parameterized traces) are compatible with its parameter instance. ${ }^{5}$ Once this has been determined, the event is checked against the monitor's property.

\footnotetext{
${ }^{5}$ Parameter instances are said to be compatible if the instance being checked is equal to or less informative than the one it is being compared against. That is, if it contains the same parameters or a subset of the parameters of the instance it is being compared against.
} 
Parametric trace slicing is therefore the mechanism by which a monitor analyzes parameterized traces and extracts only those sequences of events that have parameter instances compatible with its parameter instance.

\subsubsection{Monitor Specifications}

As has been discussed previously, a JavaMOP monitor specification contains a property, the events that may be used in that propery, and the code to handle whether or not the property is satisfied. This section will provide an overview of these various elements of a monitor specification and how they correspond to ACL elements. It will begin with the monitor header.

A monitor's header in JavaMOP contains the generic information used to control instance generation and integration. It consists of the modifiers, ID, and parameters. For the purpose of this thesis, no modifiers were used ${ }^{6}$. We are therefore only concerned with the ID and the parameters. The ID is simply the unique name for the monitor. With respect to this thesis, the IDs used consist of the name of the associated contract, whether the monitor is for a scenario or simply assertions, and whether the monitor is for any sub-scenario elements (e.g. Student_Scenario_RegisterForCourses.mop). The parameters in a monitor header have been mentioned previously and denote the set of parameters that form the parameter instances for the monitor's instances. For example, the monitor corresponding to the sub-scenario of a student taking a single course (sub-scenario of the TakeCourses scenario) has as parameters a $\{$ Student, Course $\}$ pair. Monitor instances are therefore generated for each unique pairing of a student instance and a course instance.

Following the monitor header is the body of the specification. The body contains

\footnotetext{
${ }^{6}$ Modifiers specify monitor properties. That is, a modifier is a keyword that controls the type of monitor generated. For example, defining whether a monitor is synchronized (needs to be protected against concurrent accesses) or not, or the type of indexing used for monitor indexing trees (discussed in [60] but not relevant to this thesis so will not be discussed further.)
} 
all of the event definitions, the property (or properties), and the various handlers for the events and property.

Events in JavaMOP are related to the entry and exit of actions during the execution of the IUT [56]. They are defined using a combination of modified AspectJ advice specifications and pointcuts. AspectJ advice specifications are used to specify whether the event is triggered before or after the execution or call of the method it is bound to, as well as to specify any return values to be checked. In the advice definition, the name of the event (used to identify it in the property) as well as the parameters of the event (used for the parametric trace slicing) are also defined. Following the advice specification, the pointcut defines the method the event is monitoring, whether to trigger upon the call of the method or the execution of the method, and exposes data (in the form of the parameters defined in the advice) for use in the event handler. JavaMOP pointcuts are also extended to include additional features such as the condition keyword that can be used in place of the AspectJ if pointcut and allows for the use of monitor variables in the event's pointcut definition.

An example of an event definition follows:

1 event SelectCourse after

2 (Student student, ArrayList list) returning(Course c):

3 execution $(*$ Student. SelectCourse $(\ldots))$

$4 \quad \& \&$ args(list) \&\& target(student)

The first two lines of the above event header are the AspectJ advice specification. They define an event SelectCourse that is triggered after the IUT action, has parameters of types Student and ArrayList, and returns a Course object.

The next two lines compose the AspectJ pointcut. It first specifies that the event monitors for the execution of a method (in place of a call). It does not care what the type of the method is (e.g. public, private, etc.), as denoted by the asterisk, and specifies that the method takes one or more parameters, denoted by the ".." in 
place of the methods parameter list. It next specifies what the parameters and target object of the pointcut are (using the parameters defined in the advice specification) with the $\operatorname{args}()$ and target() pointcut elements.

Following the event header in the body of a monitor specification is the event handler, or the actions to take upon occurrence of the event. In the event handler, any Java code may be used. This allows for a lot of flexibility in what JavaMOP can do. It is in these handlers that all checks, variable updates, and logging code are included (as shown in Chapter Three).

With respect to ACL, JavaMOP event definitions correspond to contract responsibilities. An event header is a translation of an ACL responsibility header, and an event handler is a translation of a responsibility's body.

Lastly in the monitor body is the property and its categories (more than one category may be defined per property) handlers. Discussed previously, JavaMOP allows for the use of a variety of logical formalisms for property definition. The one chosen for this thesis is the CFG plugin as its grammar is the most closely related to that of ACL scenarios ${ }^{7}$. The property is defined using the events previously defined in the monitor specification, and is absent any parameter values (as explained previously). At runtime, each monitor keeps track of its own parametric trace, comparing it against the defined property. Upon occurrence of a violation or match of the property, the respective handler will be executed. Much like event handlers, any Java code, as well as specialized JavaMOP keywords (illustrated in Chapter Three), may be used in these handlers.

Also important to note with JavaMOP monitor specifications is that prior to the event definitions, Java methods and variables can be defined for use within the

\footnotetext{
${ }^{7}$ Important to note is that the JavaMOP CFG plugin allows for ambiguous grammars. That is, a grammar for which there exists a non-terminal that can have more than one leftmost derivation. As such, CFGs in JavaMOP may be nondeterministic which imposes restrictions on what to test. That being said, these considerations are beyond the scope of this thesis.
} 
monitor.

\subsection{Beyond JavaMOP: JUnit}

To enforce the non-temporal checks included in ACL, a number of options were available. These consisted of either using Java if statements, or one of the Java testing frameworks available such as JUnit. As JUnit was specifically designed for software testing, it was the approach selected. This was due to the fact that it not only provided the basic assertions desired for the ACL checks, but it also included a variety of other testing features that could be used if desired. Ultimately, either approach would have sufficed for our purposes.

As mentioned above, JUnit provided the ability to use an assert method (assertTrue) to check the conditions in ACL. This assert method was used for all pre- and post-conditions, as well as any invariant checks. 


\section{Chapter 3}

\section{Example Walkthroughs}

\section{$3.1 \quad$ Overview}

The following chapter will provide a detailed breakdown of the two case studies developed for this thesis. The first is for a game of Yahtzee. It does not include much in the way of ACL scenarios, but instead serves the purpose of demonstrating the use of ACL contract variables as dynamic oracles over the execution of an IUT. The second case study is for a simple university management system. This case study is much more intensive in terms of scenarios and illustrates their more intricate semantics.

For both case studies, it is important to note that the ACL contracts were developed independent of this thesis. They were implemented with the ACL/VF tool and were tested to ensure proper functionality. The corresponding IUTs used to test the resulting mappings were however developed for this thesis based on the ACL contracts. They are minimal in functionality and only include the code necessary to demonstrate the monitoring capability of the mappings.

The main concept behind the mapping between ACL and JavaMOP is the correspondence between ACL contracts and JavaMOP monitor specifications. The goal of ACL is to provide contracts that execute alongside the IUT and can be used for runtime checking. As will be illustrated in the following examples, each contract 
corresponds to one or more monitor specifications in JavaMOP. More specifically, a contract has one monitor that handles all non-temporal assertions and one or more monitors to handle the scenarios. In other words, each element of ACL that has its own instances at execution time ${ }^{1}$ (contracts and contract scenarios) has a corresponding JavaMOP monitor. In some cases, sub-monitors are also used to handle the specialized scenario semantics such as atomic and parallel blocks (explained later).

Before getting into the case studies, the desired functionality from the VF as well as the important elements that were added to the system will be discussed. These elements are any additional features or functionality that needed to be added to our solution to be able to fully support the syntax and semantics of ACL in JavaMOP.

It is also important to note that the term "event" holds two meanings when referring to ACL and JavaMOP. In ACL, an event corresponds to a specific occurrence that can be relevant to one or more contracts, including the one where it was fired, while in JavaMOP an event is an occurrence in the IUT that is being monitored. JavaMOP events correspond to ACL responsibilities. As such, if not specified otherwise (i.e., does not specifically state ACL event), the term "event" will be referring to JavaMOP events.

For those contracts and specifications that are too long to include in the body of this thesis, the code may be found at [61]. As such, blocks of code will instead be used in the following to illustrate the examples.

The term 'mapping' in the following section refers to the development of JavaMOP code to correspond to each ACL element.

\footnotetext{
${ }^{1}$ In the following section, the term contract instance does not refer to actual instances of objects, but refers to the parts of a contract that had instances in the VF tool. In this thesis, monitors correspond to contract instances. The term contract instance is used for ease of understanding.
} 


\subsubsection{Validation Framework Functionality}

The VF tool takes as input three elements. The first of these is a TRM expressed using ACL. This was discussed previously in Section 2.1 and will not be explained further.

The second input element taken by the VF is the candidate IUT. This is the implementation that the ACL will be executed against. As the development of an IUT is outside of the scope of this thesis, it will not be discussed except for any elements necessary to illustrate the mappings between ACL and JavaMOP.

The third and final element taken by the VF is the binding information. For the VF to be able to properly execute the contracts against the IUT, the types, responsibilities, and observabilities from the contracts must be bound to concrete implementation artifacts located within the IUT [10]. As mentioned previously, this was handled through the use of Microsoft Phoenix in the original VF tool. The development of such a binding tool for the Java environment is outside of the scope of this thesis however and is being completed as part of a separate Master of Computer Science degree. All bindings in the following case studies were done manually.

As mentioned previously, the VF tool conducts runtime verification by executing ACL contracts against an IUT execution. This means that while an IUT is executing,

the VF tool is able to track and record execution paths generated by the IUT (used to verify each scenario execution matches the associated grammar of responsibilities), while also executing any dynamic checks [10].

\subsubsection{The Blackboard}

The blackboard system is a set of classes to be included with any ACL/JavaMOP system. This set consists of two classes. The first, called Blackboard, is a singleton that contains the necessary methods to fire and observe events, as well as the data 
structures to keep track of the mappings between contract classes and their respective IUT instances. The second class, called ACLEvent, is used to store ACL event information such as ID, context, and data.

As a system, these classes act as a blackboard to which any ACL events can be fired. In other words, all monitor specifications that fire or observe ACL events, or that require some form of communication between monitors, use the blackboard. It functions through the use of method calls made through the Blackboard class, namely a call to the Blackboard's fire method. This call may take as parameters either an instance of the ACLEvent class, or the individual data members of the ACLEvent class (i.e., the fire method is overridden to accept three parameters where each parameter corresponds to a data member in the ACLEvent class). This call to the fire method will then be detected by any monitor that contains event definitions for one or more ACL event occurrences. More specifically, the events that are fired through the blackboard are not actually stored or recorded by the blackboard. Instead, it is the invocation of the fire method that is monitored.

The blackboard is also used to keep track of each monitor's associated contract class (described later). This is accomplished through the use of a map data structure that maps IUT class instance IDs to their corresponding contract class instances. That is, each contract has a corresponding data structure in the blackboard that maps IUT instances to contract class instances. As such, the blackboard system must be tailored to the system being tested. That is, since different systems will have different numbers of contracts, the blackboard class will need to be generated with the appropriate number of map data structures for the number of contracts. It cannot be simply a static system that is universal to all projects. 


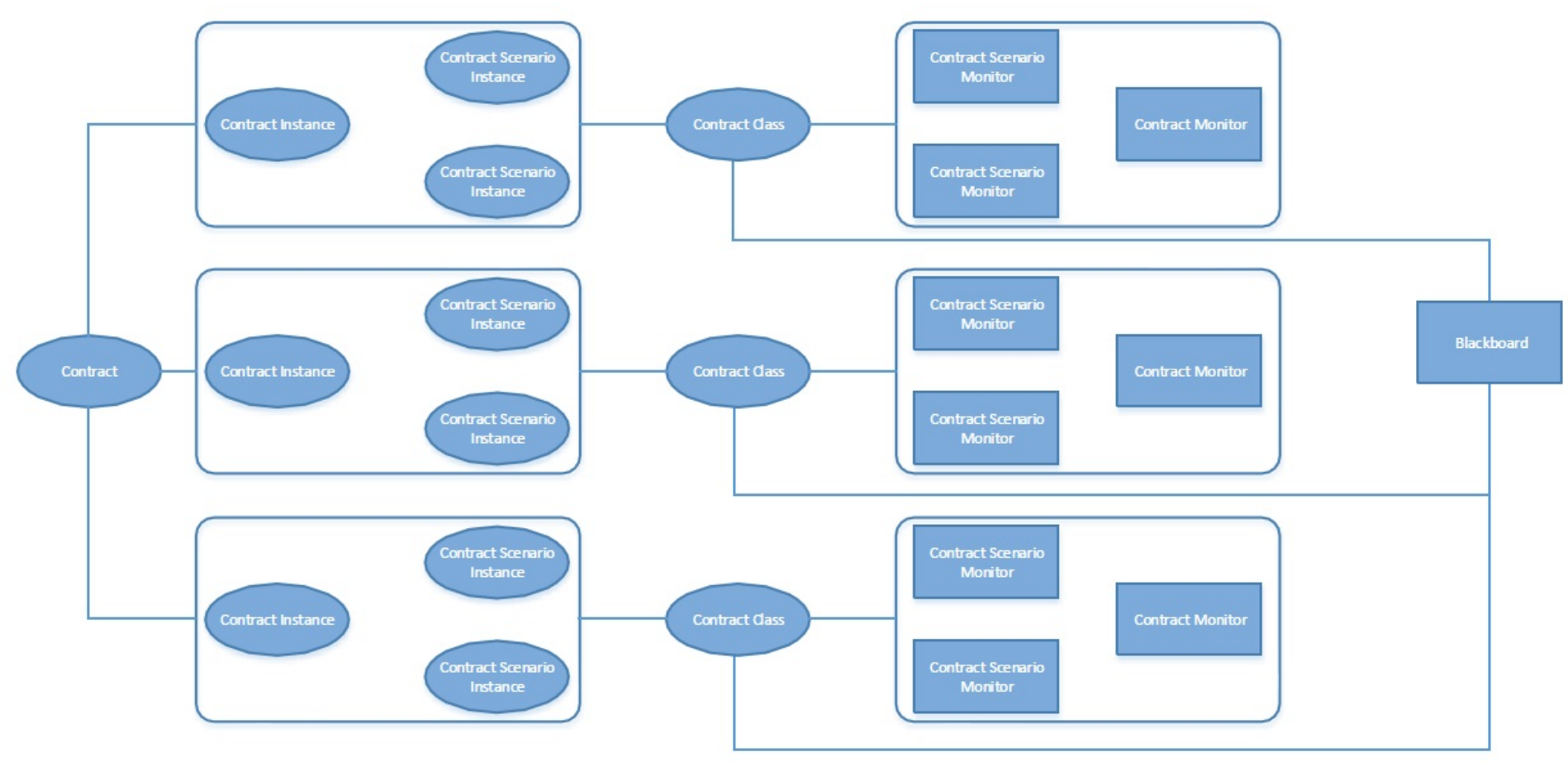

Figure 2: Relationships between system elements.

\subsubsection{Contract Classes}

Contract classes are used to provide a central location for contract-scoped elements so that they can easily be accessed across monitors. Each contract has a corresponding contract class that includes the contract variables, observabilities, and invariant checks for that contract. As IUT instances are created at runtime, the contract class, corresponding to the contract the instance is bound to, is also initialized. The mapping between the IUT instance and its corresponding contract class is then stored by the blackboard.

The observabilities and invariant checks are included purely for organization purposes. That is, instead of redeclaring the necessary observabilities and invariants in each monitor specification that uses them, the contract class provides a central location that the monitor instances may reference.

Since there may be multiple monitors in existence for a single contract, there 
needed to be some way to make contract-scoped variables accessible across these monitors. For example, in the university case study, the Student contract has three monitor specifications associated with it. As such, contract-scoped variables such as the number of failures needs to be accessible by all Student contract monitors. This was accomplished by including it in the Student contract class and adding a reference to this class in each student monitor. This way, all monitor instances for a single student instance can access and update the variable. Important to note is that a contract variable is specific to the monitors for an instance of the contract, not static across all instances. So, there is a new contract class created for each instance of Student in the IUT, each having its own instance of the number of failures variable.

Figure 2 provides a graphical illustration of the relationship between contract classes and other ACL/JavaMOP elements. In this figure, each oval or rectangle (different to separate which element of the mapping they belong to. i.e. ACL or the JavaMOP mapping) represents some entity from either ACL or the mapping to JavaMOP. As well, each group contained within a border represents multiple entities originating from a single entity. For example, a contract instance and the corresponding contract scenario instances all originate from a single contract specification. Lastly, the lines connecting the various elements represent associations between the elements they connect. For example, a collection of a contract instance and its corresponding contract scenario instances are associated with a monitor and the scenario monitors, and both of these collections are associated with a single contract class.

As illustrated in Figure 2, each contract may have one or more contract instances (and their sub-instances) associated with it. These contract instances correspond to JavaMOP monitors. Each instance has a corresponding contract class instance, managed by the blackboard. For example, an instance of a Student object has one or more corresponding contract instances (monitors) associated with it. It also has a corresponding contract class instance. The blackboard maintains the mapping 
between the object instance and its contract class instance through the use of a map data structure. This map takes object IDs as keys and contract class instances as values.

\subsubsection{Enhanced ACL Events}

Originally, events in ACL consisted solely of an ID. As such there was no way to transfer any additional information with an ACL event. That is, there was no way to specify the context of the event (i.e. from what contract the event originated), or any additional data that may be relevant to the event.

Our solution therefore includes enhanced events that not only have an ID, but also a context and data object. These additional attributes are generic object types and as such, may carry any type of information. In general however, the context attribute carries the instance reference of the IUT object the monitor that generated the event is bound to.

These enhanced ACL events will be demonstrated in the following university case study.

\subsection{Yahtzee Case Study}

The following Yahtzee example is one of the first iterations of the mapping from ACL to JavaMOP. It does not include much of the refinement that will be found in the university example. This is due to the fact that for the purposes of this example, said refinement was not necessary. As such, the monitor specifications for the Yahtzee game include all variables, invariants, and observabilities in the same specification as their corresponding responsibilities. It does however include the blackboard described above. 


\subsubsection{Significant Elements}

The first example discussed is a pair of responsibilities from the Yahtzee project's main ACL contract.

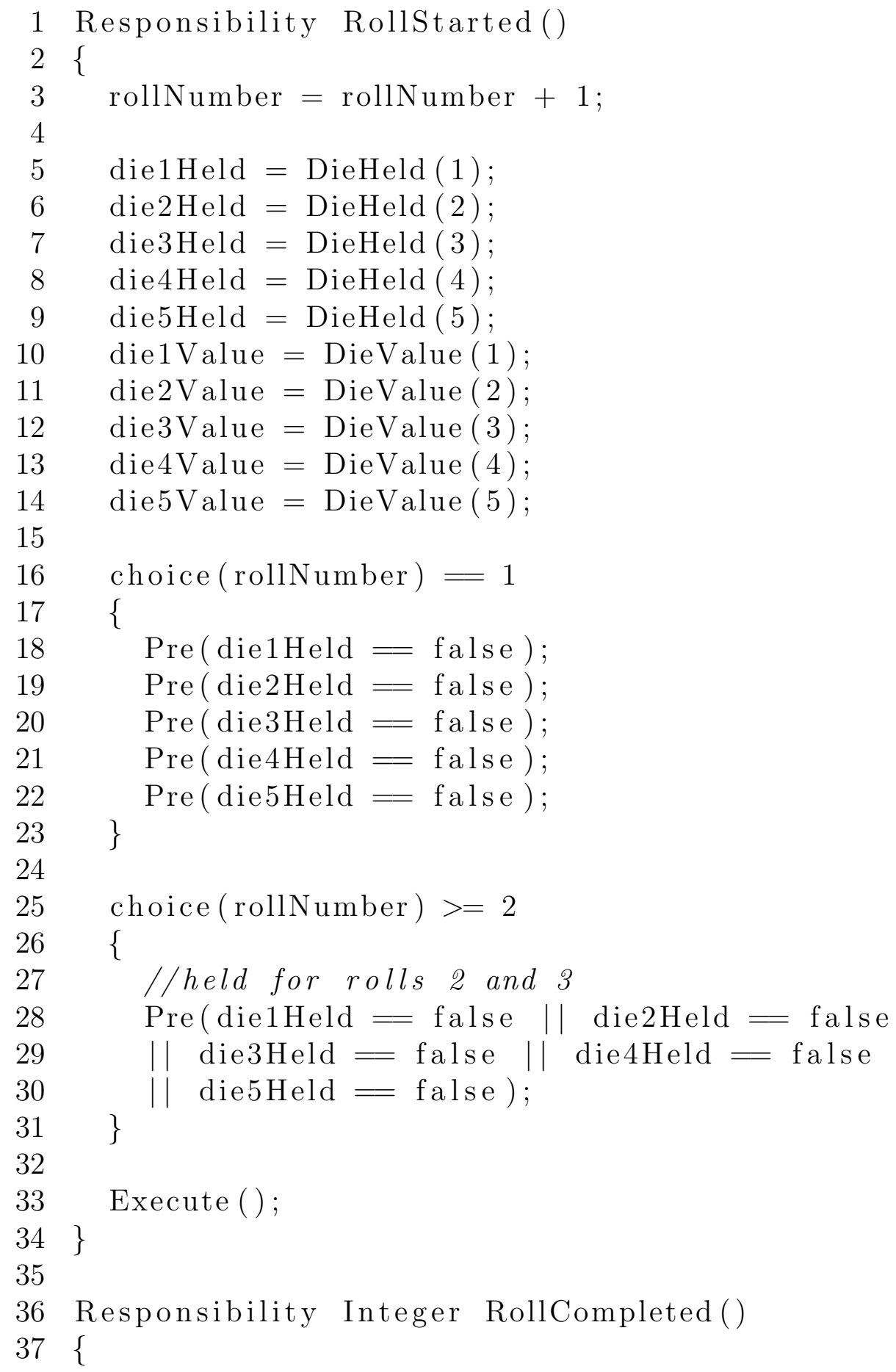




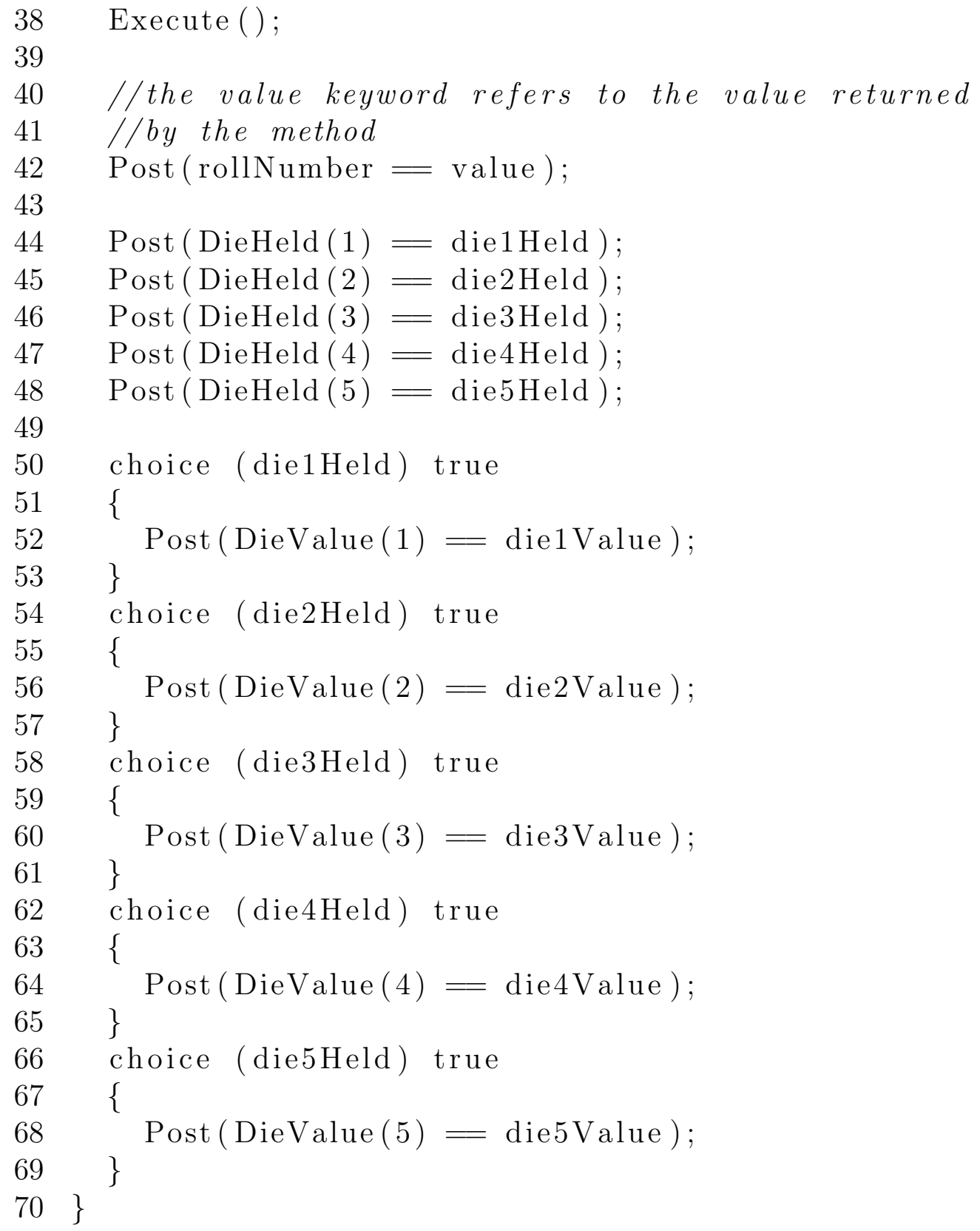

The above responsibilities demonstrate a number of ACL elements. Some elements have been discussed previously, but can be seen in better detail here. These are elements such as the pre- and post-conditions, and contract variables. In the above two responsibilities, one can see how contract variables, such as die1Held and die1Value, can be used as test oracles. As illustrated in the code, the values are 
initially set in the RollStarted() responsibility, where they use their corresponding observabilities, parameterized by the die number, to retrieve the die information from the IUT. That is, die1Held is set based on whether die one is held in the IUT before the roll, and die1Value is set to the pre-roll value of die one in the IUT. Pre-conditions are also used to check that, on the first roll (lines 16-23), no dice are being held, or if not the first roll (lines 25-31), that no more than four dice are being held.

The second responsibility uses the values of the previously set variables to check conditions after the roll. That is, it ensures that any dice held prior to the roll are still held after and that the values of those held dice did not change during the roll.

As one can see, in place of requiring test oracle values to be pre-determined, ACL provides a solution where the necessary test values are determined during execution.

The following code is the corresponding JavaMOP events for the above responsibilities.

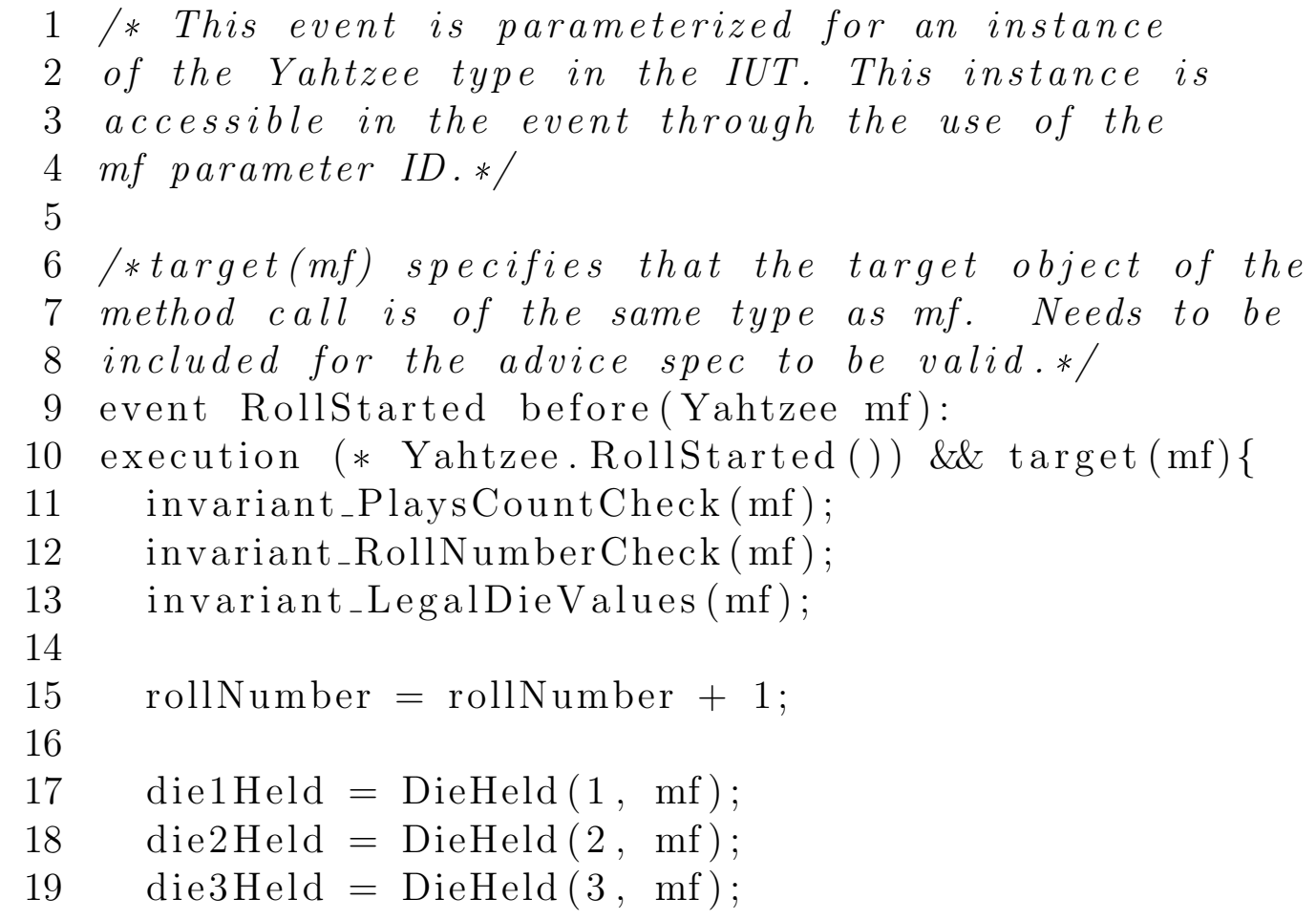




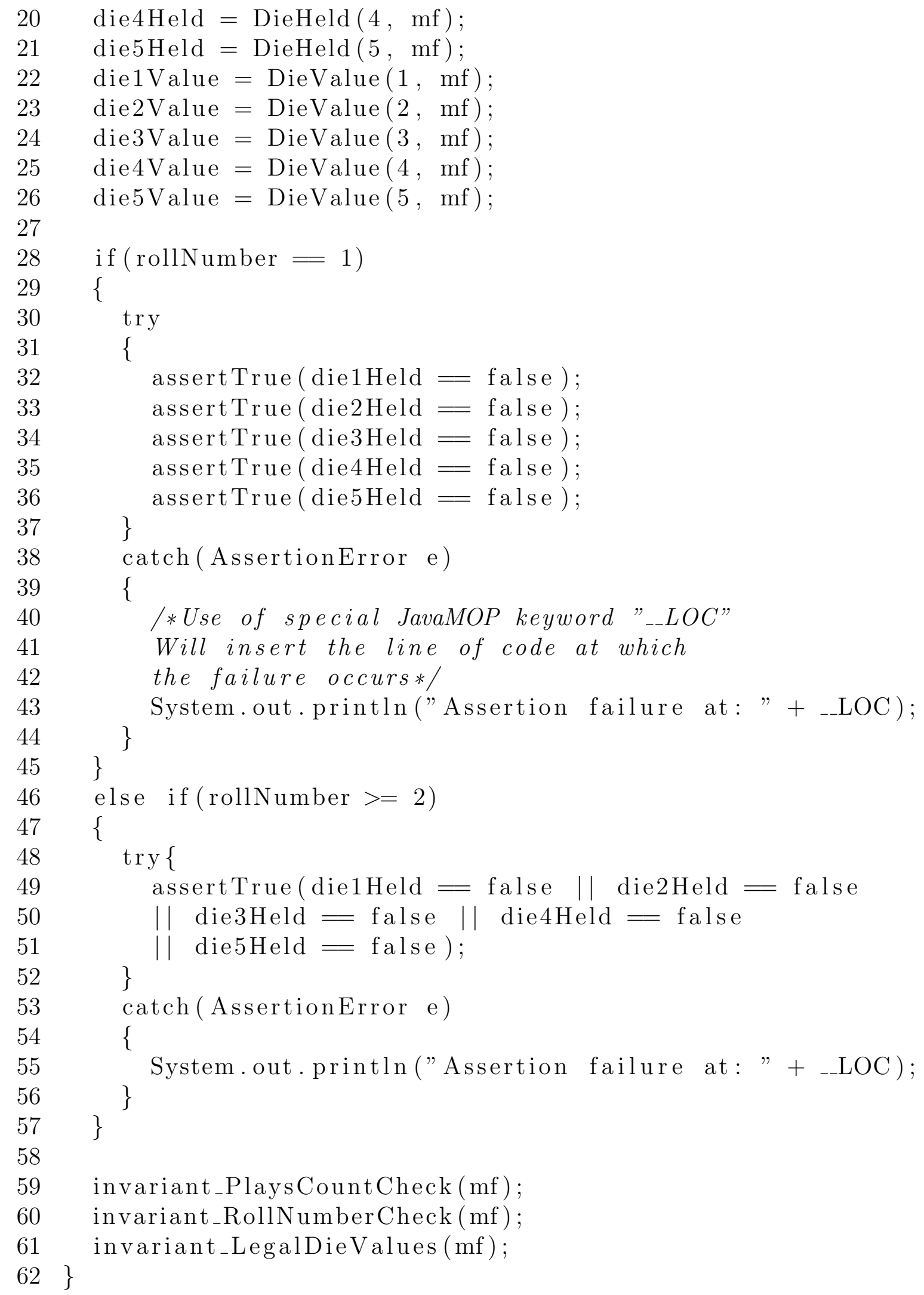




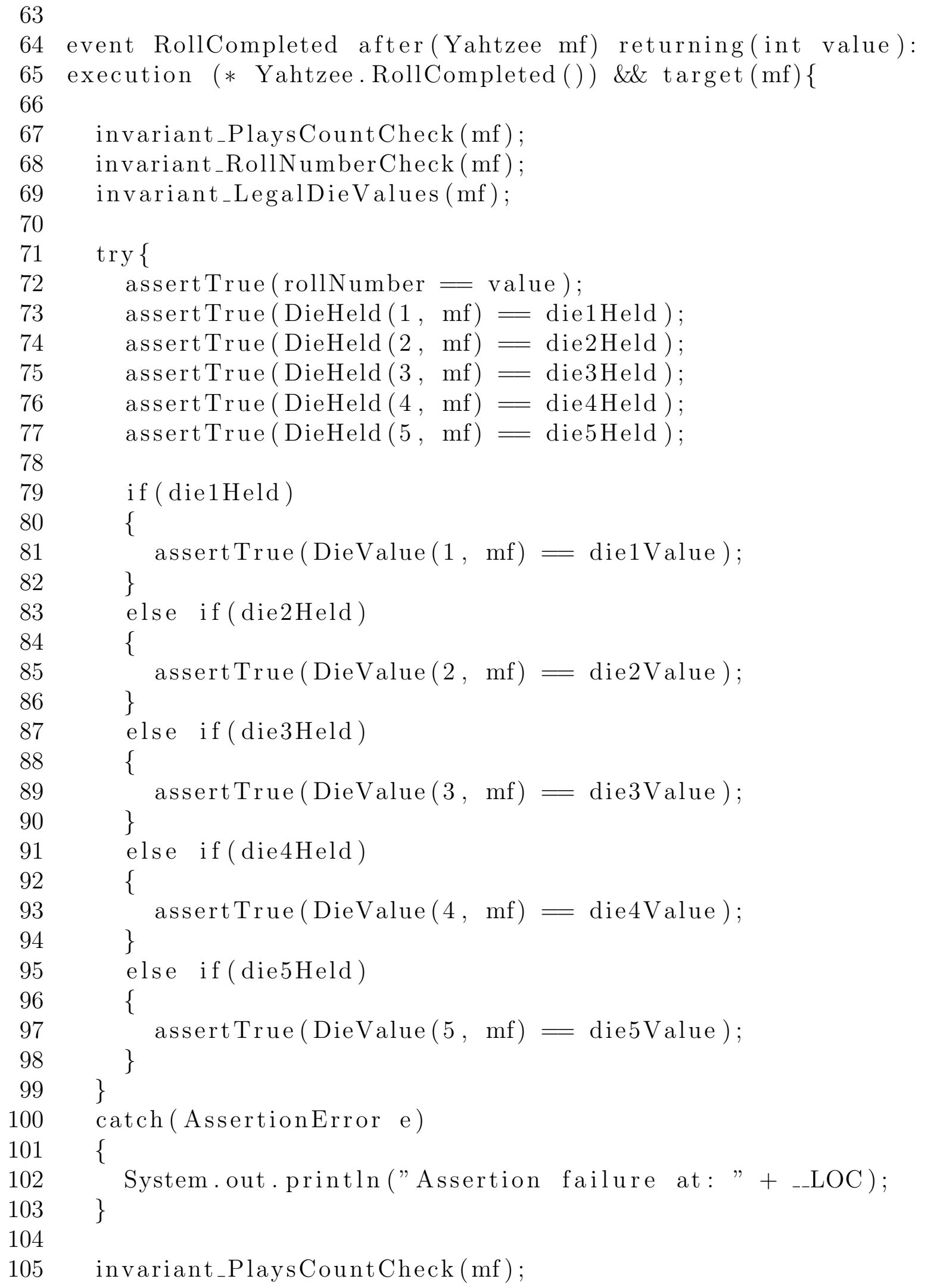


106 invariant_RollNumberCheck (mf);

107 invariant_LegalDieValues (mf);

$108\}$

As one can see, in terms of conditions and assertions, excluding the temporal aspects, there is almost a direct mapping from ACL to JavaMOP. The variables are set in the RollStarted event (lines 9-62) and then used in JUnit assertions (i.e. assertTrue()) in the RollCompleted event (lines 64-108) to check the post conditions. One can also see how invariants are called at the beginning and end of the events corresponding to the responsibilities. These checks are implemented as Java methods defined in the monitor's specification.

Another key point in the above example is the use of the Java try and catch blocks. These allow checking of the conditions without termination of execution in case of a failure. This type of handling could be used to record logs of event occurrences in an actual implementation of an ACL/JavaMOP tool.

For further examination of the Yahtzee game and its ACL to JavaMOP mapping, see $[61]$.

\subsection{University Example}

The university case study is significantly more complex than the Yahtzee one above. In this second case study, the focus was on the mapping of the temporal aspects of ACL into JavaMOP. Namely, the scenarios. As this was the most significant feature of ACL to be mapped, the university case study was the central effort of this thesis. As such, the following will provide an overview of the resulting mapping as well as a discussion of the difficulties that were overcome to generate the mapping.

The key ACL scenario elements and concepts that needed to be handled in the mapping to JavaMOP (some of which were discussed previously in Chapter Two) were as follows: 1) The various types of iterations available in a scenario. This includes 
loop structures as well as constraints on responsibilities in a scenario grammar. 2) Atomic blocks where only those responsibilities included in the atomic section, and in the order specified are valid. No other responsibilities from the contract may occur while execution remains in the atomic section. 3) Parallel blocks where multiple instances of the parallel section may be active simultaneously.

All of the above, along with additional ACL elements, will be illustrated in the following code examples taken from the case study.

\subsubsection{Significant Elements}

Before getting into the details of the various semantics of the ACL scenarios of the university case study, we will examine a contract in its entirety and discuss the various ACL elements not provided in Chapter Two. Following this discussion will be the corresponding JavaMOP monitor specifications. The contract used for this example will be the Course contract taken from the University Example originally provided by Arnold [62]. The original ACL contracts may also be found on the site for the appendices of this thesis at [61].

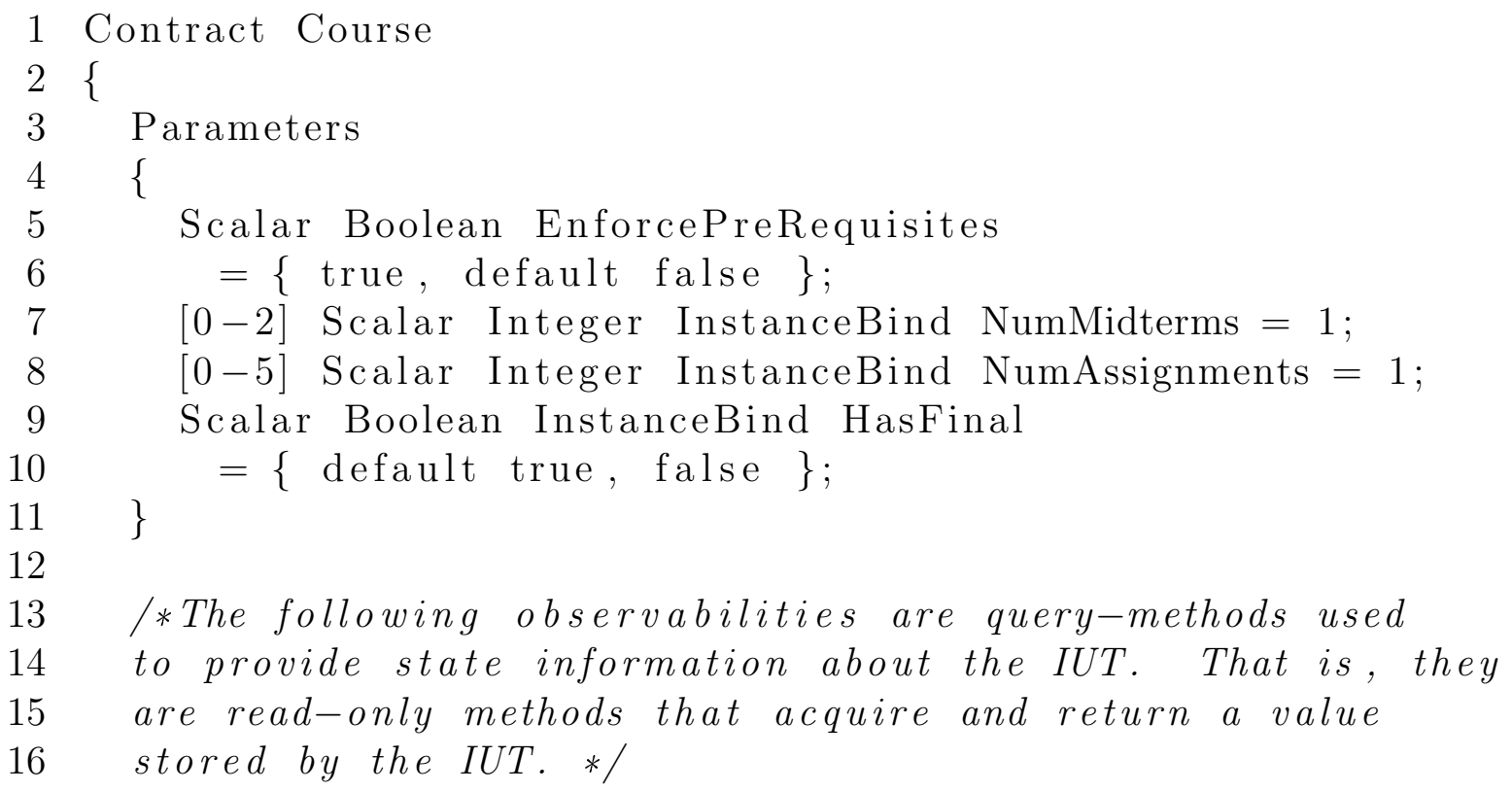


17 Observability String Name();

18 Observability Integer Code();

19 Observability List tStudent Students();

20 Observability Integer CapSize();

21 Observability List Integer PreRequisites();

22 Observability Integer

23 AssignmentWeight(Integer assignmentNum);

24 Observability Integer MidtermWeight(Integer midtermNum);

25 Observability Integer FinalWeight();

26 Observability Integer MarkForStudent(tStudent student);

27 Observability Boolean HasFinal()

$28 \quad\{$

29 Parameters. HasFinal $=$ true;

$30 \quad\}$

31 Observability Integer TotalMarks()

32\{

33

51 Observability Boolean IsFull()

52\{

Scalar Integer markTotal $=0$; loop(1 to Parameters. NumAssignments) \{ //the counter keyword is part of the ACL syntax //it is used to keep track of the number of iterations //of a loop \} markTotal $=$ markTotal + AssignmentWeight $($ counter $) ;$

* This observability gets the taken courses from the

56 student contract $s$ and checks that all of the prerequisites

57 have been taken by the student.*/

58 Observability Boolean HasPreRequisites (tStudent $\mathrm{s}$ ) 59\{ 


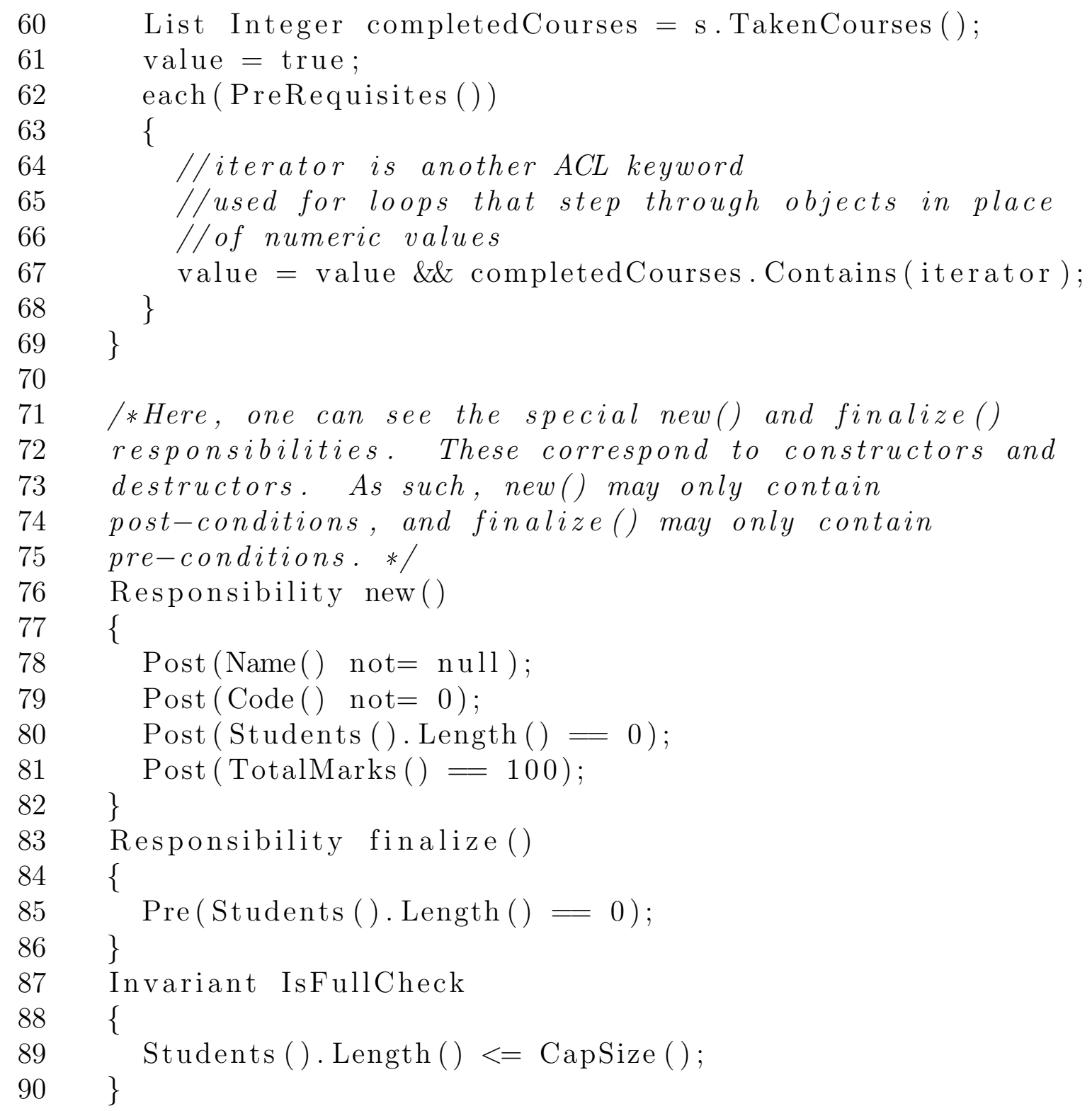




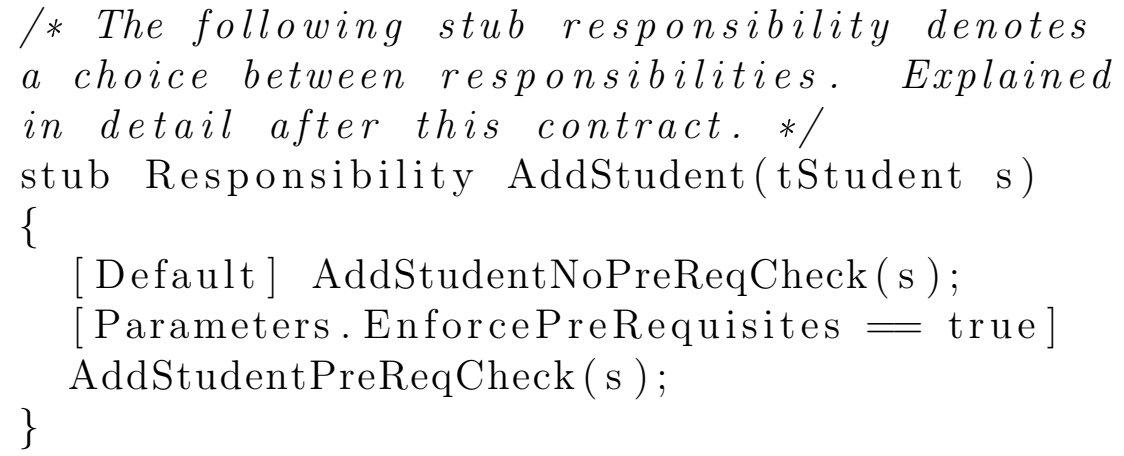


44

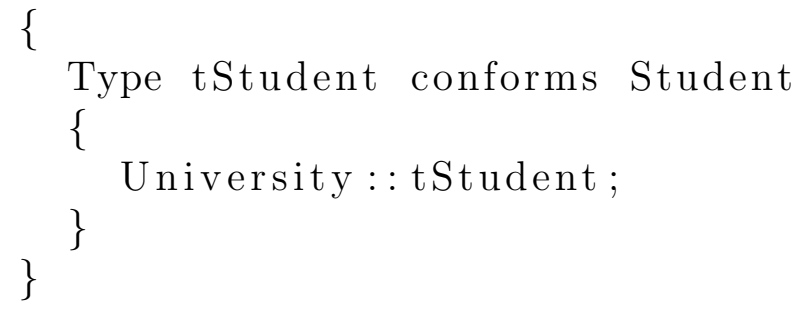

This excerpt includes elements such as pre- and post-conditions, invariants, observabilities, and responsibilities. While these will not be discussed further, the above ACL does contain examples of some implementations of these elements not provided previously. For example, the contract includes unbound observabilities such as TotalMarks() where parameters, contract variables, bound observabilities, and other language constructs are used to calculate and return a value in place of directly querying the IUT.

In addition to the previously discussed elements, the above contract also contains ACL elements not yet mentioned. These are parameters and responsibility stubs on which we briefly elaborate below.

Contract parameters are used to configure the contract. That is, they can be used to specify different scenario paths, or simply specify a constant value. These values are determined at binding time. If the parameter declaration uses the keyword InstanceBind (lines 7,8, and 9), then each contract instance has its own value for that parameter. Otherwise (line 5), the parameter is static across all instances of the contract. Parameters can be seen as analogous to testing parameters in other testing tools [15]. In the Course contract, parameters are used to specify required properties of the course such as the number of midterms and the number of assignments.

Responsibility stubs are analogous to stubs found in use case maps [63]. That is, they represent a point where different functionality can be placed into the location represented by the stub. In the case of the AddStudent(tStudent s) (lines 49) stub, this functionality takes the form of whether or not a prerequisite check is 
necessary before adding a student. The decision on which action to take is determined at runtime based on the conditions in the stub.

Finally, the Exports section of an ACL contract serves the purpose of sharing types across contracts. This is not relevant to this thesis and so will not be discussed further.

In the next section, we present the JavaMOP monitors that correspond to the above ACL contract. An important point of note is that there may be one or more monitor specifications for each contract. This will be discussed in more detail in further examples, but the main concept is that for each element of a contract that may generate an instance, there is a corresponding JavaMOP monitor specification. As well, there is a corresponding contract class (described above) for each contract. This will not be included in the following code as it is simply a Java class that contains the aforementioned methods and variables corresponding to the contract observabilities, invariants, parameters, and contract variables. It does however contain an example of how InstanceBind can be handled. It is included in Appendix A for self-containment and completeness.

The first monitor provided corresponds to all of the non-temporal assertions in the contract. It handles the pre- and post-conditions, runtime checks, and invariants.

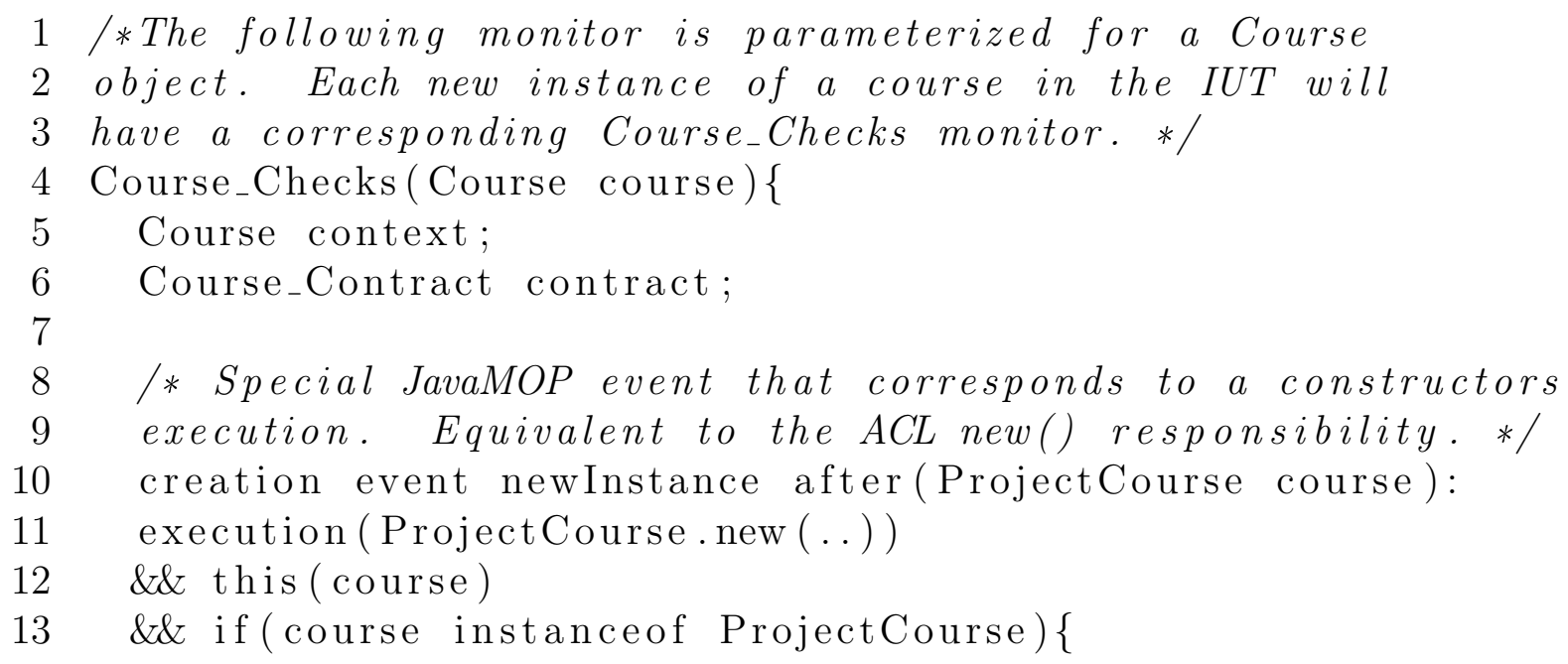


/*This is where the contract class associated with the IUT instance is created and added to the blackboard. */ if (Blackboard. CourseInstances. get ( course.toString ()$)=$ null) 
$57 \quad \& \&$ args (student)

58 \&\& target (course) \{

64 after(Course course, Student student):

65 execution (* Course.AddStudentNoPreReqCheck $(*)$ )

$66 \quad \& \&$ args (student)

$67 \quad \& \&$ target (course) \{

68 contract.invariantChecks ();

71 event AddStudentPreReqCheck

72 before(Course course, Student student):

73 execution (* Course.AddStudentPreReqCheck $(*)$ )

$74 \quad \& \&$ args (student)

$75 \quad \& \&$ target (course) \{

82 after(Course course, Student student):

83 execution (* Course.AddStudentPreReqCheck $(*)$ )

$84 \quad \& \&$ args (student)

$85 \quad \& \&$ target(course) \{ 
100

101

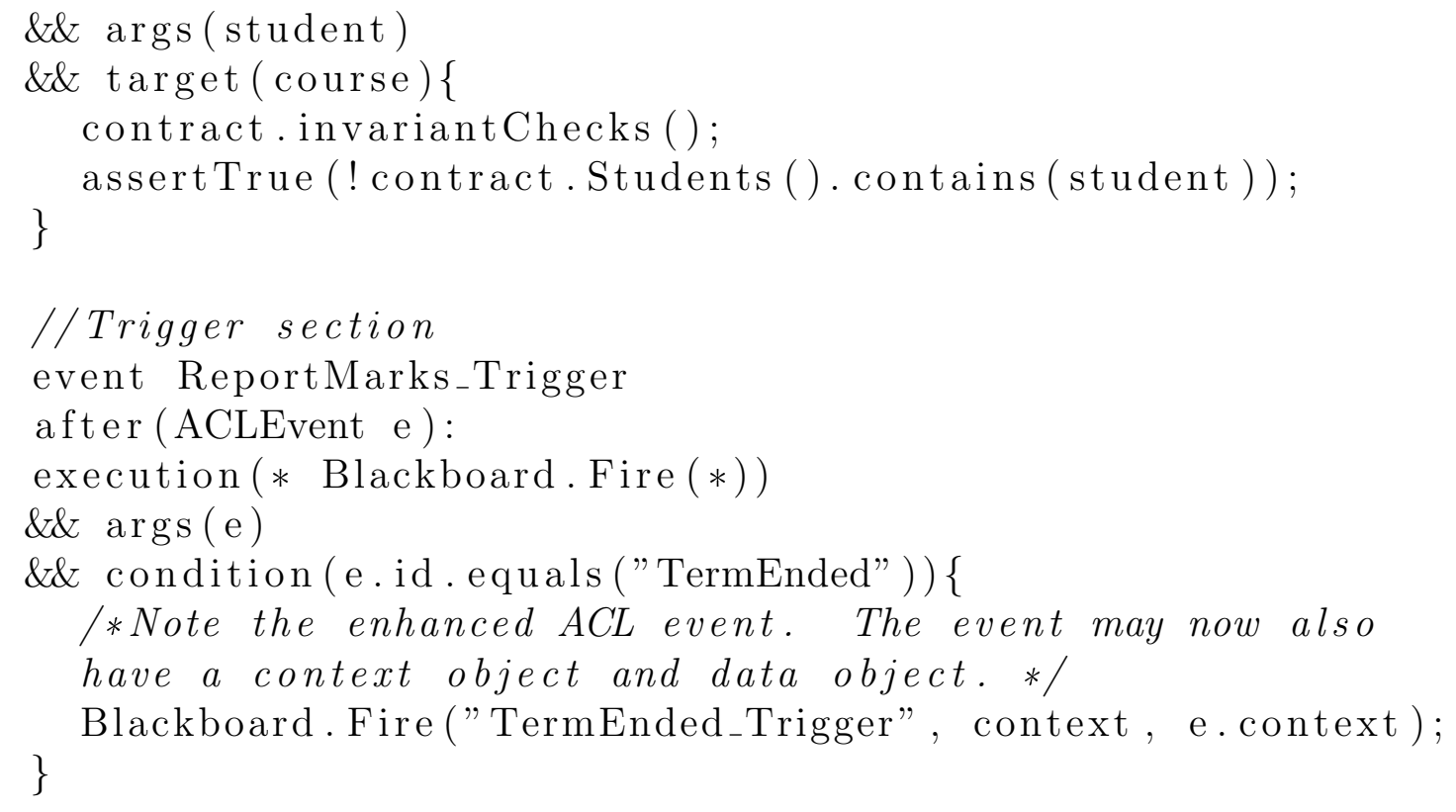

The above code illustrates the handling of an important aspect of ACL that has not yet been discussed. That is, the support of contract inheritance. The Course contract has an associated ProjectCourse contract that inherits from it (See [61]). This is handled in the above monitor through the use of multiple newInstance events where each such event generates the appropriate contract class. Further experimentation late in the case study revealed that the most recent update to JavaMOP now supports inheritance $^{2}$. However, due to time constraints, the changes to our code required to use this new feature have not yet been carried out and are left as future work. Ultimately, these changes would provide cleaner, more efficient monitors, but the functionality would remain the same.

Also illustrated above is how we handle pre- and post-condition checks in JavaMOP. AspectJ's around advice was not implemented correctly in JavaMOP event

\footnotetext{
${ }^{2}$ The version we are using (3.0) claims to support this. However, due to a lack of documentation on this functionality and apparent bugs in its implementation, we could not establish how it is used
} 
definitions and as such could not be used. This was discovered during experimentation. Proper implementation of this type of advice would have allowed us to implement both pre- and post-conditions in a single event, exactly like in ACL responsibilities. This is because around advice allows for the specification of actions to take prior to execution, when to execute the bound method, and the actions to take after execution. To work around this bug, AspectJ's before and after advices are used. As one would assume, the before events are used to handle all pre-execution code while after events handle post-execution code. This includes all checks, conditions, and invariants. The only additional change necessary when using two events in place of a single event is that any variables that are declared within a responsibility, prior to execution, must be made monitor-scoped variables so that they may be used across events.

Beginning from the top of the above monitor specification (line 10) is the creation event. Creation events in JavaMOP are the only events that can trigger the creation of new monitor instances. As such, these events correspond to the creation of a new object instance (in the case of assertion monitors), and the trigger conditions in the case of scenario monitors. Also included in the creation events is the code necessary to create associated contract class mappings in the blackboard (lines 17-25). This code first checks to see if a mapping already exists for the IUT instance the contract is bound to. If no such mapping exists, the contract will create one and add it to the blackboard.

Following these are the add students events. Both of these events have corresponding responsibilities, as shown in the contract code, but also must handle the AddStudent(tStudent s) stub (lines 59 and 76). This is done by including the conditions of the stub into each event. In the above example, this is handled through the use of a JUnit assertion, but could also be handled as a condition in the event itself. 
The final section to discuss in the above monitor specification is the trigger section at the bottom of the specification (lines 107-115). This section is used to initialize all scenario instances that are not directly triggered by the creation of a new instance. That is, all scenarios that are dependent upon some specific occurrence for initialization. This is handled through the use of special triggering events that monitor for the trigger condition. Upon occurrence of this condition, the triggering event will use the blackboard to fire an ACL event ${ }^{3}$ that will initialize the appropriate scenario monitor instance. In the case of the above code, the monitor will watch for the occurence of the ACL event TermEnded (line 107-111). When this ACL event is fired, the monitor will recognize its occurrence and in turn fire an event called TermEnded_Trigger (line 114) that will initialize another monitor responsible for monitoring the ReportMarks scenario.

The next block of code is that of the monitor specification corresponding to the Course contract's scenario: ReportMarks.

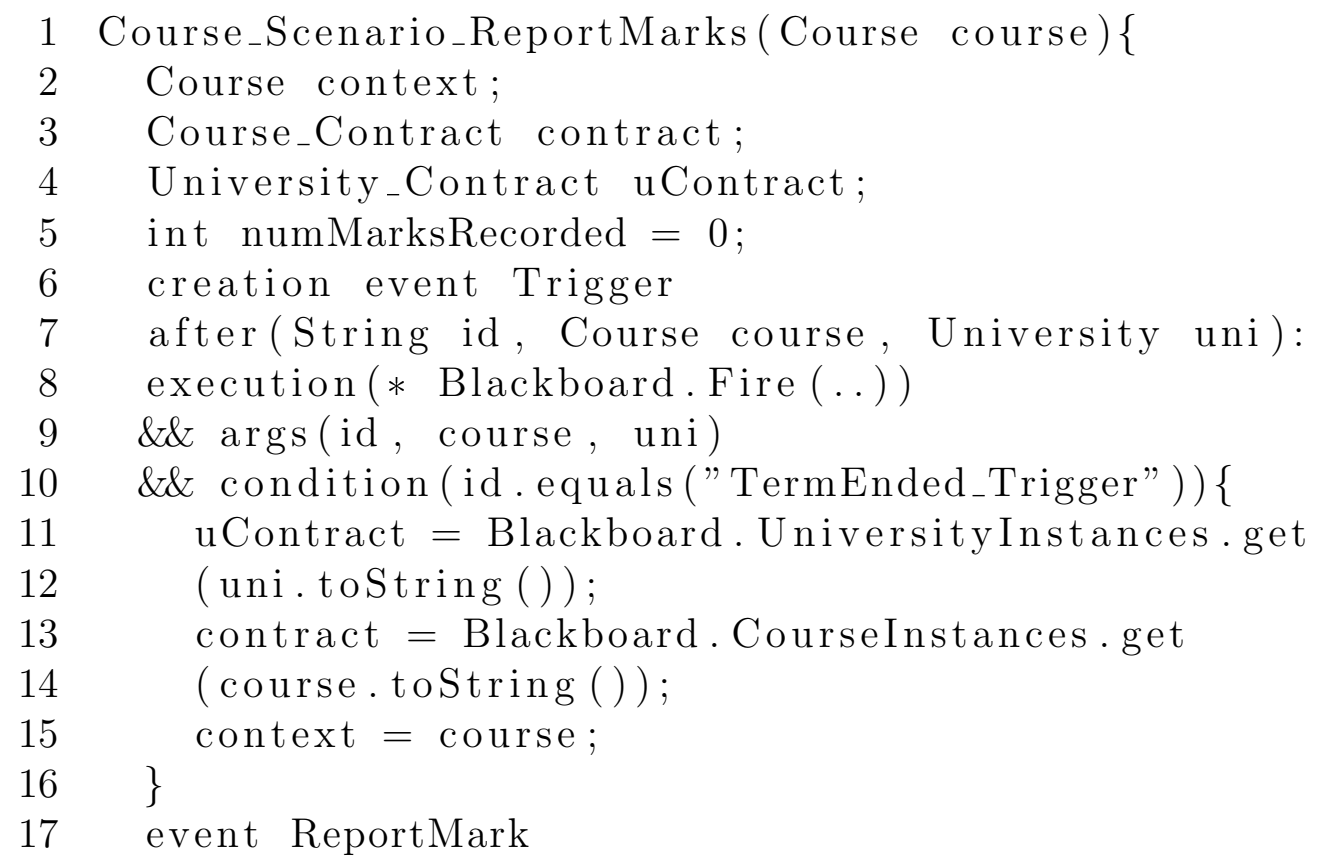

\footnotetext{
${ }^{3}$ As mentioned previously, blackboard events can also be used for communication between monitors, without any relation to the ACL contracts.
} 
after(Integer mark, Course course, Student student): execution (* University.ReportMark (..))

As illustrated in the above monitor specification, scenario monitors contain the same responsibilities as the contract's Checks monitor. However, the responsibility definitions in the scenario monitor are absent any of the non-temporal assertions (i.e. pre- and post-conditions, invariant checks).

The above specification also provides an example of how a loop is handled in the mapping. This is the each(Student()) loop from the contract. There are two aspects that need to be addressed to be able to handle this loop structure. The first is the actual looping functionality. This is handled through the monitor's property, defined with a CFG. The Kleene star on the ReportMark event (line 36) denotes 
that the event may occur zero or more times before the next event in the sequence. The second aspect of the loop structure is the restriction on the number of times the event may occur. Since there is no way to specify such a constraint from within the logical formalism (CFG), a workaround needed to be used. This workaround was to count the number of times the ReportMark event occurs and check this number before allowing the next event to occur. That is, in the definition of the following event (the Terminate event), a condition is added (line 32) that only allows that event to be recognized if the number of iterations of the previous event matches what was defined in the contracts loop structure. This is done by firing the parameterized terminate event on every occurrence of the ReportMark event. With the above condition, the terminate event will therefore only be recognized when the ReportMark event count is at the right number.

Also shown above is how a property match or failure can be handled. In the ReportMark scenario, a failure results in an exception being thrown, indicating that the scenario grammar was violated. A match on the other hand simply resets the monitor, waiting for the Trigger event to be fired. As this thesis is a feasibility study only and not an implementation of an actual tool, the code in the handlers is included simply to illustrate the type of functionality available. Depending on what is desired in the final tool, this functionality may change.

The next two examples to be discussed are the most significant of this thesis. They illustrate the complexities of ACL scenarios and how they may be handled with JavaMOP. The first example will be the RegisterForCourses scenario of the Student contract, followed by the TakeCourses scenario of the same contract.

The following is the ACL scenario specification for RegisterForCourses.

1 Scenario RegisterForCourses

2\{

3 Scalar tCourse course; 


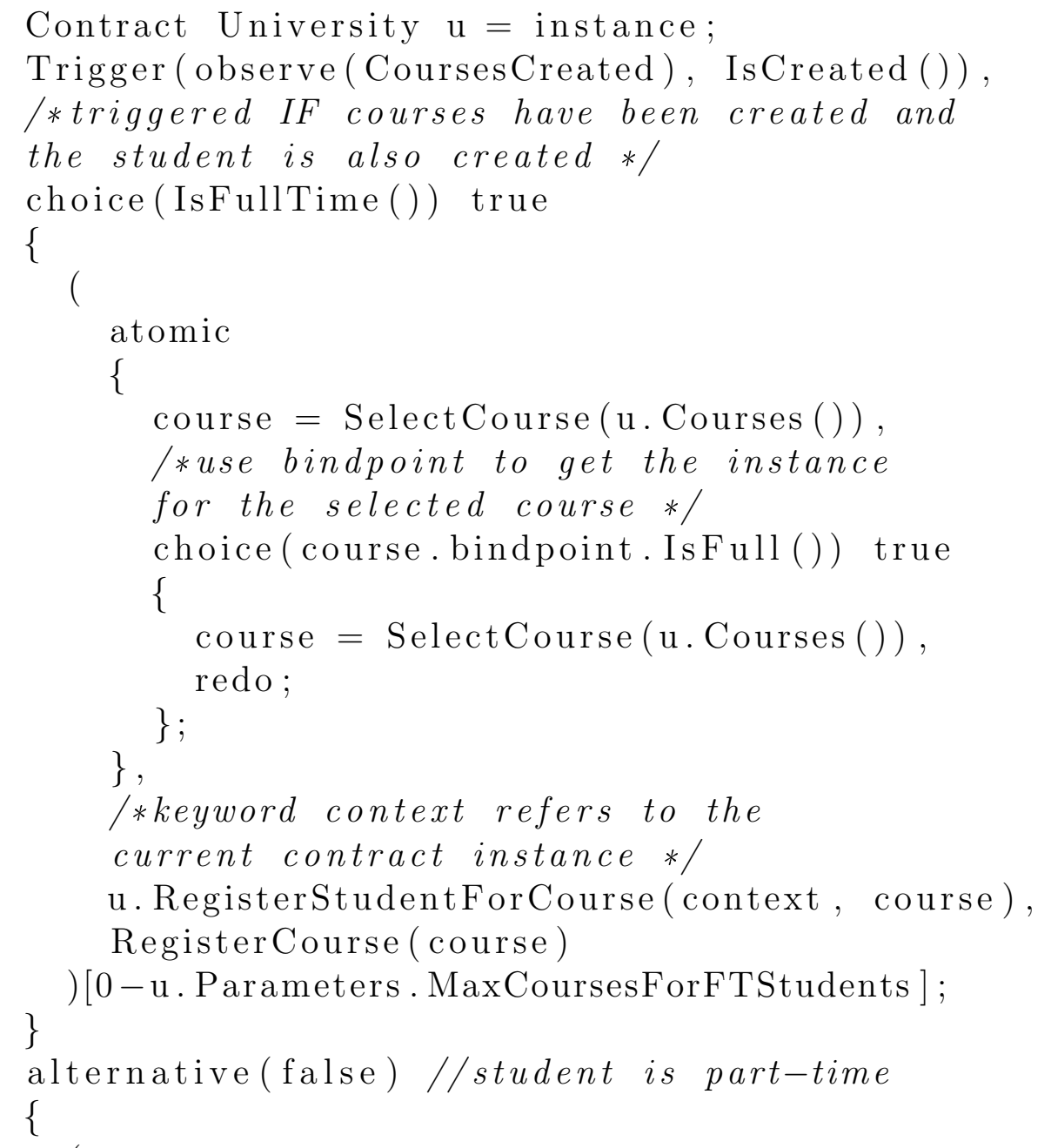


The above scenario defines the sequence of responsibilities involved in a single student registering for his or her courses. As such it handles the possibility of that student being either full-time or part-time. This is handled through the use of the alternative keyword (line 28). In the above scenario, the alternative path simply changes the maximum number of courses a student may register in.

Also illustrated in the above example is the use of repetition operators (lines 26 and 42). These are constraints on blocks or statements in a scenario. There are a number of different types of repetition operators of ACL that can be used (discussed in Chapter Four). Those in the scenario above are range operators and are located at the end of each alternative block. Their use in the above scenario also provides an example of an issue in the current ACL syntax we discovered while developing our mapping. That is, the use of a range operator immediately prior to an empty Terminate() statement. This combination results in an unknown termination point. More specifically, an empty terminate denotes that the scenario may end immediately upon reaching the Terminate() statement. When the prior condition is a range repetition operator, there is no way to determine at what iteration the scenario should terminate. For example, if the range is $0-5$, it is not clear whether the scenario should terminate when the count is at 2 , or wait until the count is at 5 . The solution we used is illustrated in the following example code. Specifically, in the condition of the Terminate event of the Scenario_RegisterForCourses monitor where it includes the checks: numRegistered $==$ uContract.MaxcoursesForFTStudents and numRegistered $==\mathbf{u C o n t r a c t . M a x c o u r s e s F o r P T S t u d e n t s . ~ I n ~ p l a c e ~ o f ~}$ allowing the number of iterations to be a range, we have fixed it so that if the student is full-time, the number of registered courses must equal the number of courses a full-time student is allowed to enroll in and, if part-time, likewise but for a part-time student. 
The last significant element of ACL in the example above is the use of the atomic statement. As was mentioned previously, an atomic section in ACL indicates that only the responsibilities within the atomic block, and in the specified sequence are considered valid. When not in an atomic section, any responsibility not directly specified in the scenario may occur without triggering a violation of the scenario. In an atomic section however, if any of the responsibilities from the contract occur, they are checked against the grammar.

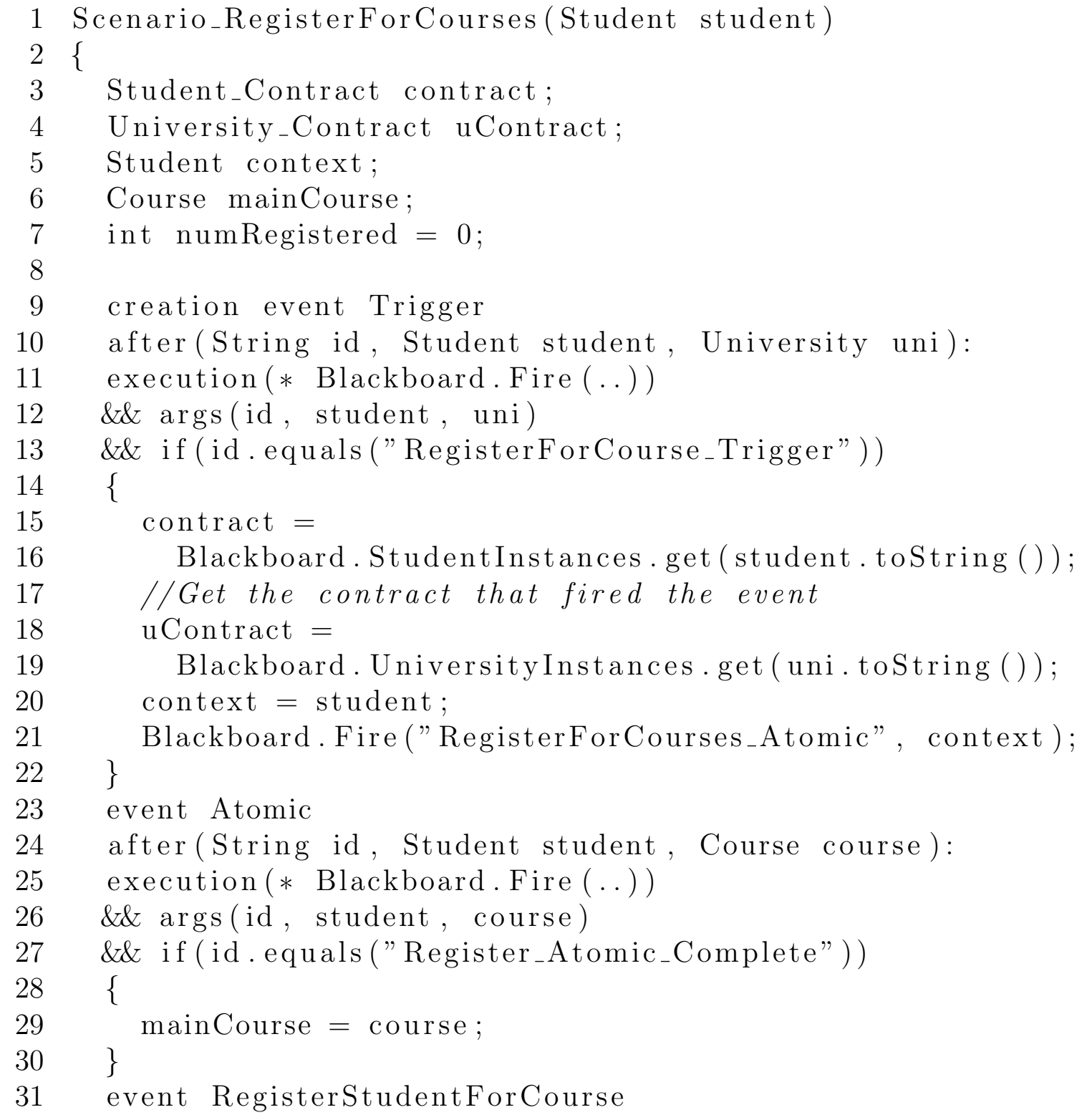




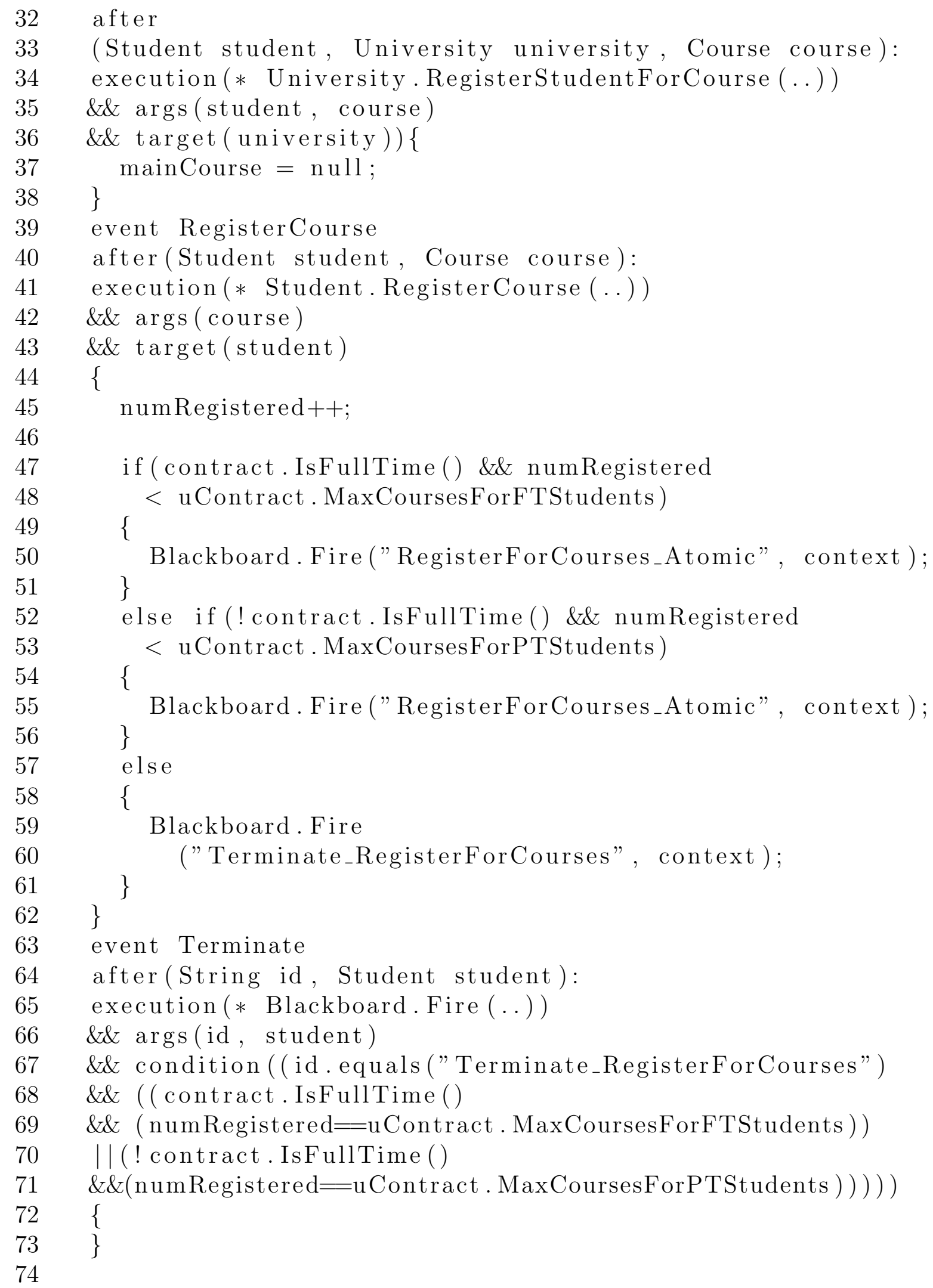


//epsilon is used in the following CFG to indicate the empty string. cfg: $\mathrm{S} \rightarrow$ Trigger LOOP Terminate,

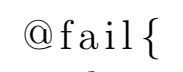




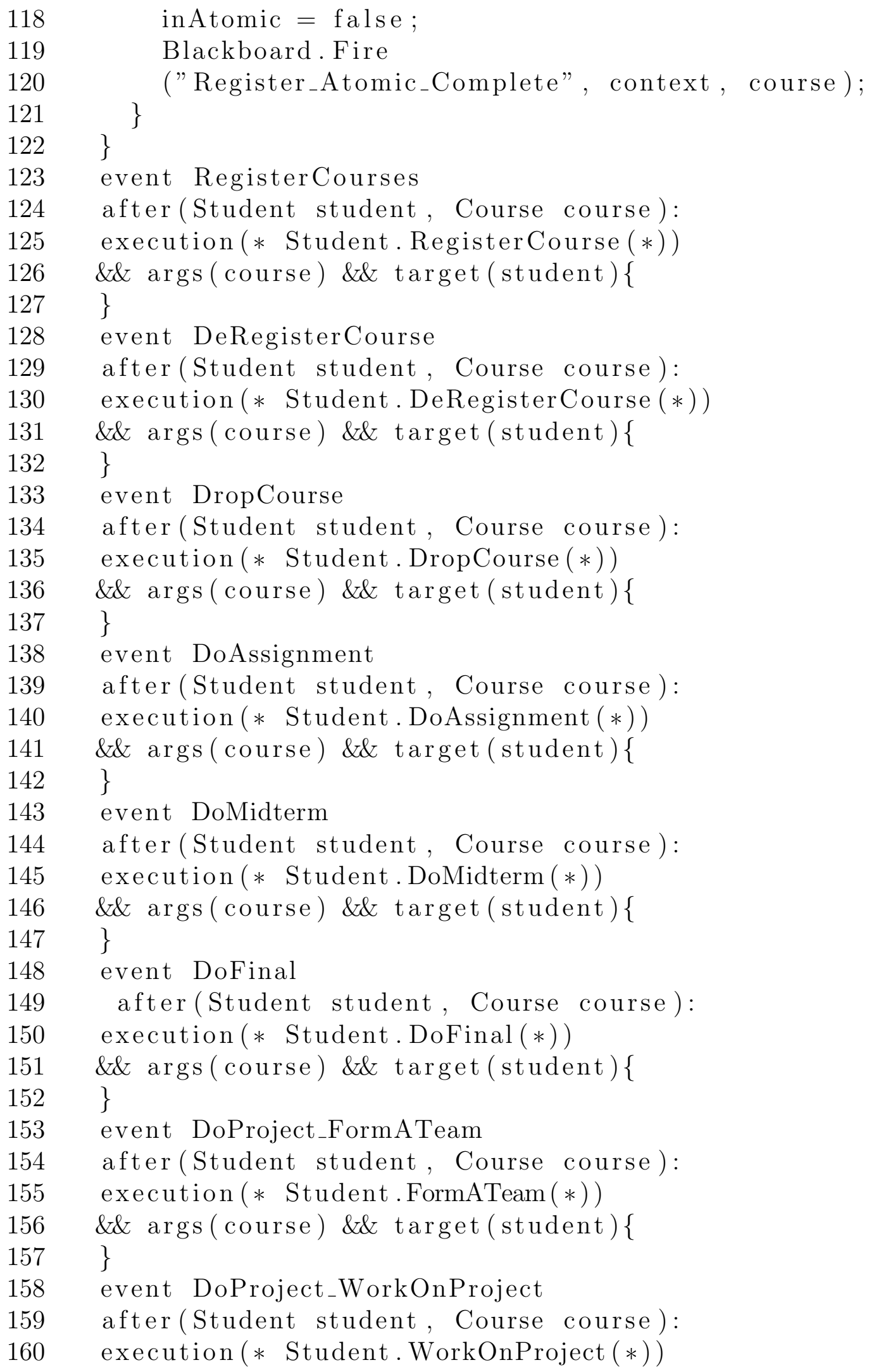


161

162

163

164

165

166

167

168

169

170

171

172

$181\}$

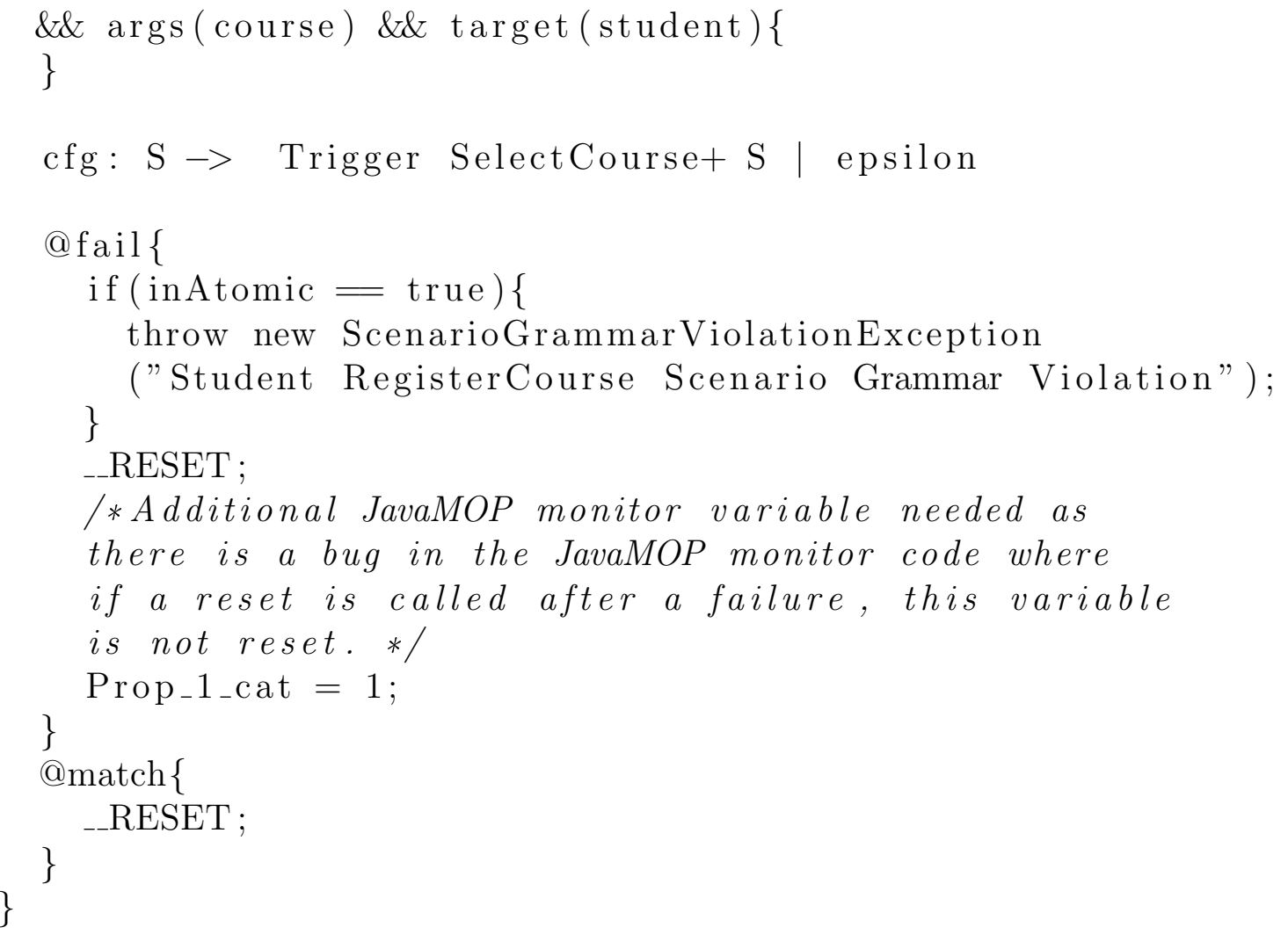

As shown by the above monitor specifications, two monitors are used to handle the single RegisterForCourses scenario. This is done to handle the atomic section.

The first monitor specification is the parent, containing the majority of the scenario. That is, it contains the events to trigger and terminate the scenario, as well as the logic necessary to handle the alternative block. In general (i.e. in an implementation of these mappings), an OR branch would be used in the logic property to define both paths defined. However, due to the difference between the paths only being the repetition operator, a condition in the final event was used instead. This condition checked the count based on the status of the student. As well, to avoid the issue of not knowing when the scenario should terminate, as mentioned previously, a fixed operator was used to determine if the scenario is complete. This can be seen in the condition on the terminate event (lines 69 and 71). 
To handle the atomic section, a second monitor is used. This is so that all of the responsibilities of the contract may be defined as events without impacting the non-atomic sections of the scenario. While all responsibilities are included, only those actually in the scenario have any event actions and are included in the grammar. All other corresponding events are simply definitions included so that their occurrence will be recognized by the monitor (lines 123-162). As such, once initialized, the atomic monitor will detect the occurrence of any responsibility from the contract. It will then check that the event is in the grammar, and that it is the next valid event. Since the monitor will not be immediately destroyed upon completion, a flag is also necessary to indicate when the monitor is active (line 95). That is, since we do not know when the garbage collector will remove the instance of the atomic monitor, it may remain active after execution leaves the atomic section. Normally, this would not be an issue. However, the above atomic monitor is part of a loop in the scenario and so needs to be reset upon completion, waiting for execution to possibly re-enter the atomic section. This means that while execution is not in the atomic section, the atomic monitor is still actively waiting for events. As such, the flag is used to effectively disable the monitor (line 118) until the triggering condition is received again.

The final significant element contained in the scenario is the obtaining of a university contract instance (lines 18 and 19). The same algorithm used in the VF (found in $[15])$ is used in the above monitor to accomplish this. Namely, the section of the algorithm that handles a single contract instance. In the case of the above contract, only a single instance of the university will ever be in existence. As such, no searching needs to be done and the monitor can simply retrieve the one instance from the blackboard. However, in a case where more than one instance exists, the binding tool would be needed to prompt the user for the appropriate instance.

The next example is the most complex scenario in the university case study. It incorporates atomic sections, parallel sections, OR statements, and an alternative. 
Due to the complexity of the scenario, it will be briefly summarized and illustrated graphically. It first begins with the trigger condition being an observance of the TermStarted event (line 4). Immediately following, a parallel block is defined (line 5) where each parallel instance corresponds to a course currently being taken by the student. Then, for each of these courses, an atomic block denotes the valid actions a student can take (line 9). These actions consist of doing assignments, midterms, a project, and a final (all dependent on the constraints contained in the '[' and ']' following each responsibility). At this point we will also point out an error in the ACL contract that was not caught by the VF when written. This is the parallel block within the atomic block (line 12). The ACL specification document [63] specifically states that "Atomic elements cannot be nested nor can they contain parallel elements.". It is assumed that the specification document is correct, therefore this nested parallel section was ignored for this case study.

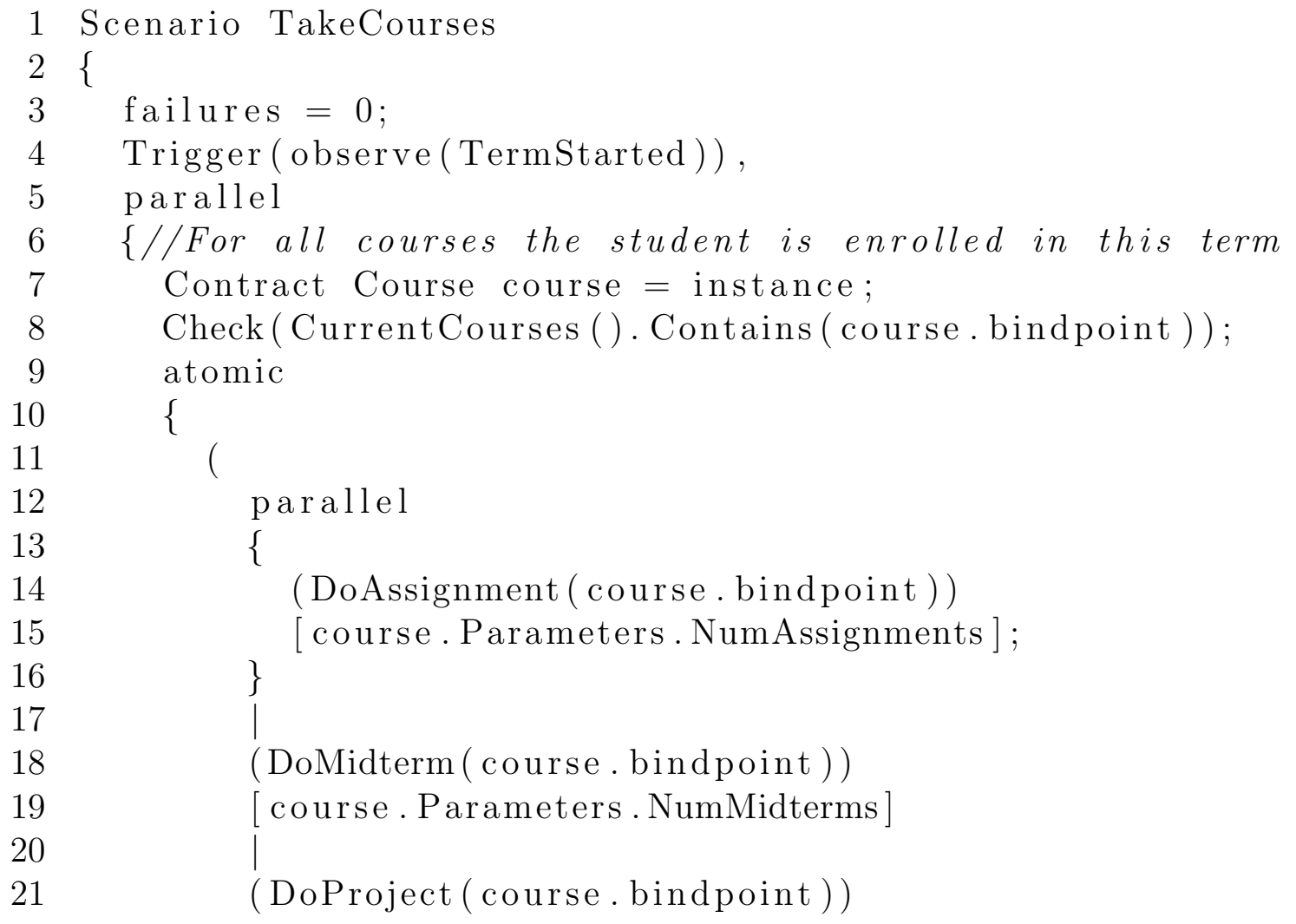




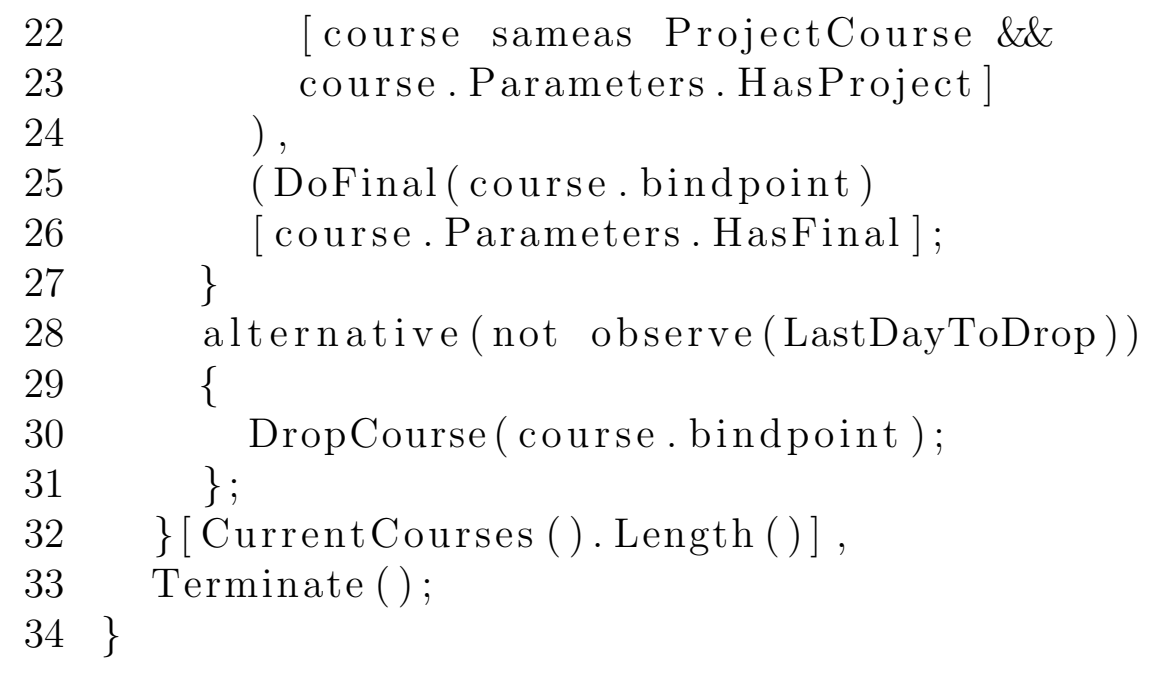

Since the atomic and alternative elements have been discussed and illustrated previously, the two elements left are the parallel block and the OR statement.

The parallel block denotes a section of a scenario that may have multiple simultaneous instances executing. In the above example, this translates to a single student taking the courses they are enrolled in. So, if a student is enrolled in four courses, when execution reaches the parallel block, four sub-scenario instances, each corresponding to a single course, need to be created for that student.

The $\mathbf{O R}$ statement, denoted by the | character (lines 17 and 20), indicates that the elements may occur in any order. There is however some ambiguity in the description of how ACL handles this OR statement. By the traditional understanding of an OR, only one of the elements needs to occur before execution may continue. In ACL however, the claim is that all elements still execute, and the OR simply denotes that their executions may be in any order. As such, it is arguable that the above OR statement should also be in a loop, iterating until all elements have satisfied their constraints. For the purpose of this thesis however, it will be treated as described in [15]. Namely, that all responsibilities in the OR statement must execute the specified number of times before execution may continue.

1 Scenario_TakeCourses (Student student) \{ 


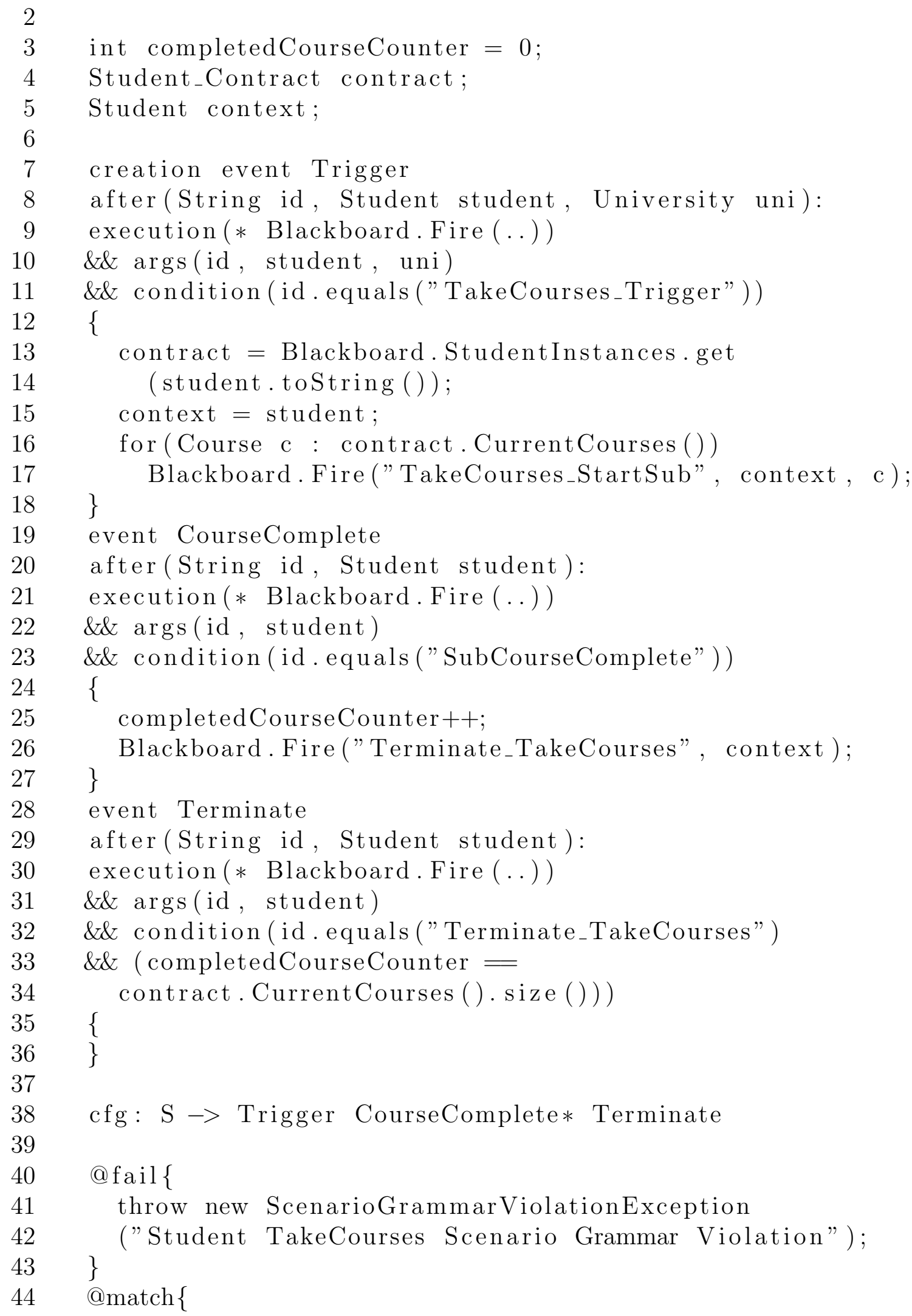




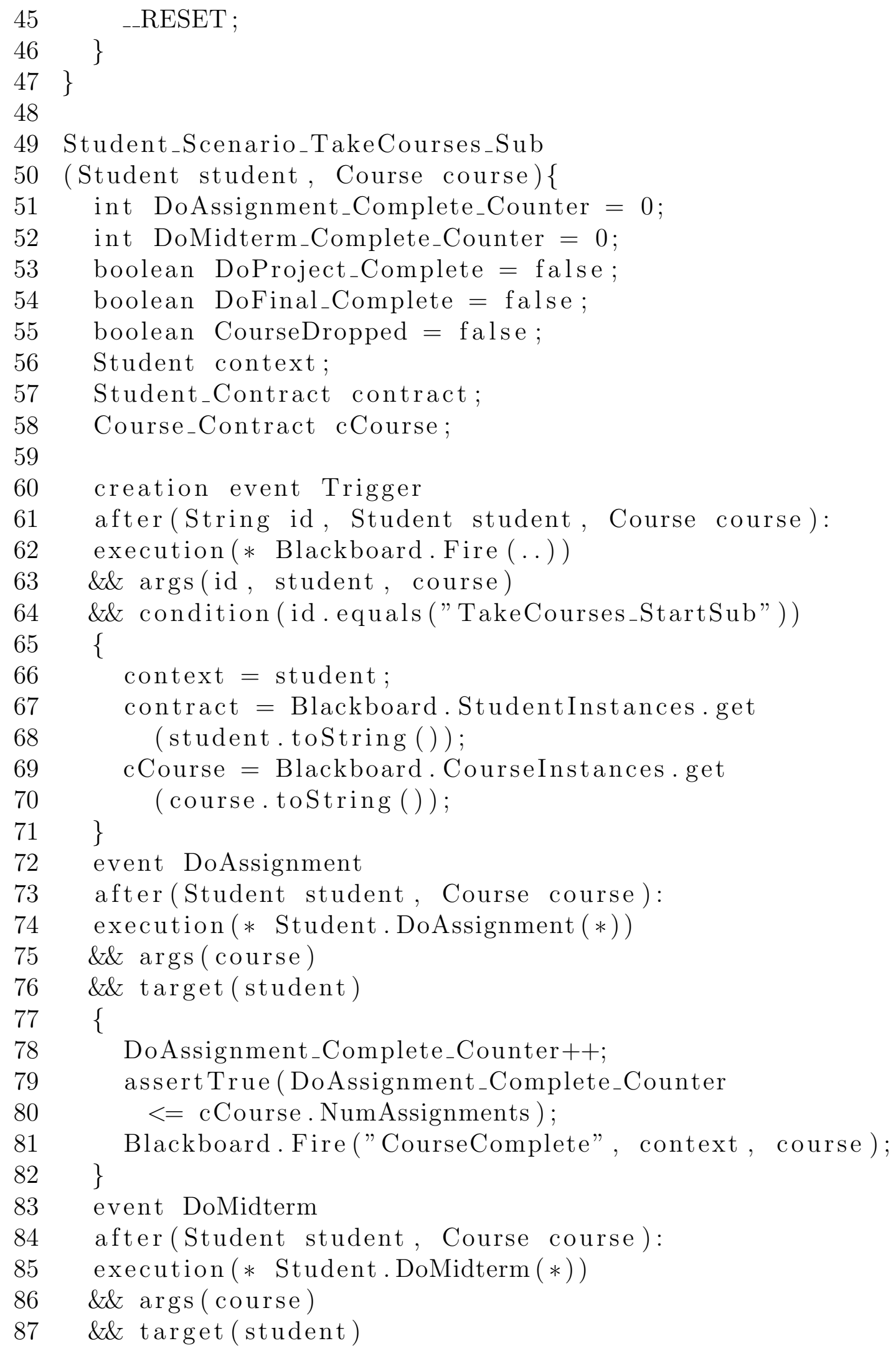




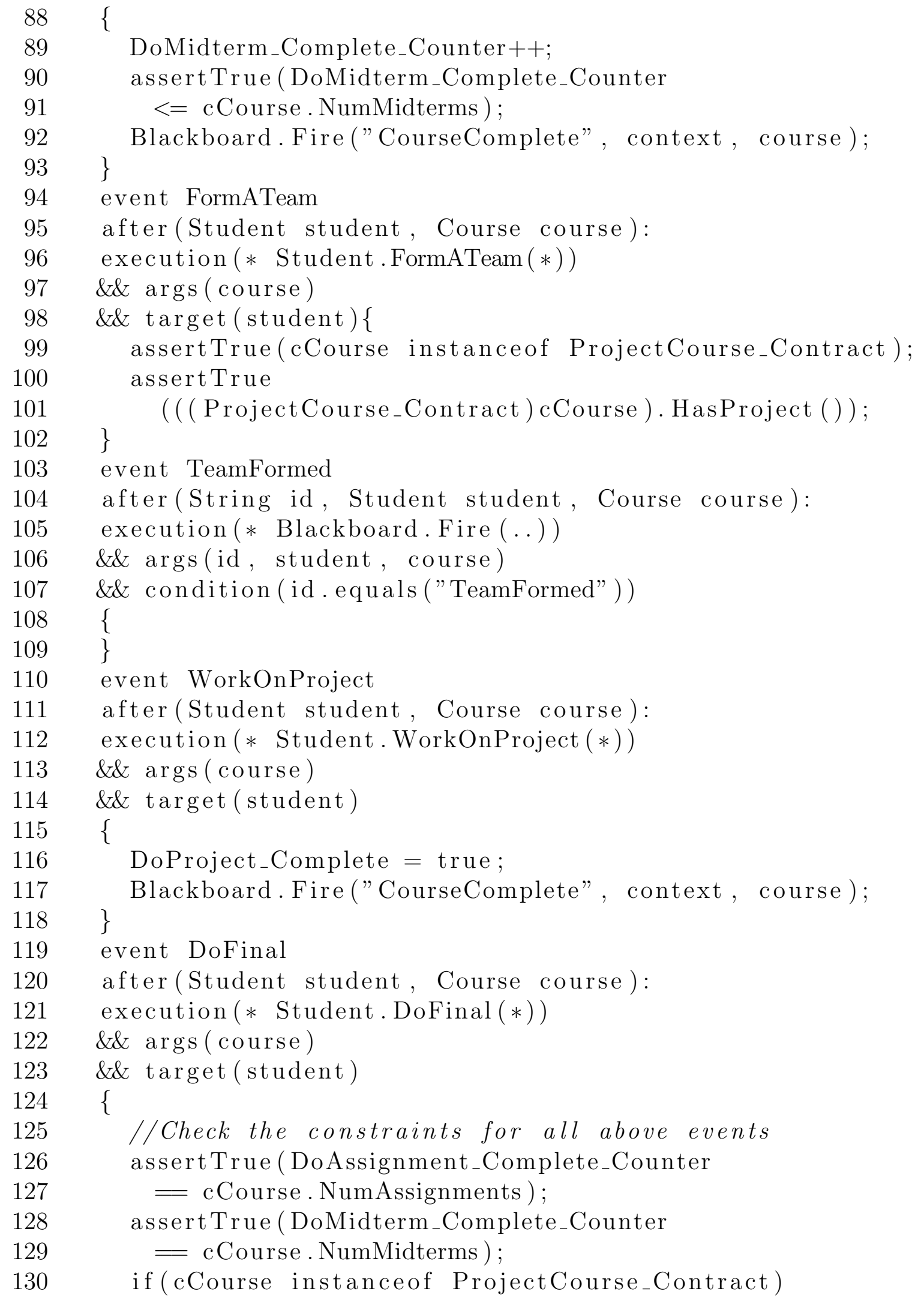


131

assertTrue (DoProject_Complete); assertTrue (cCourse. HasFinal ()); 


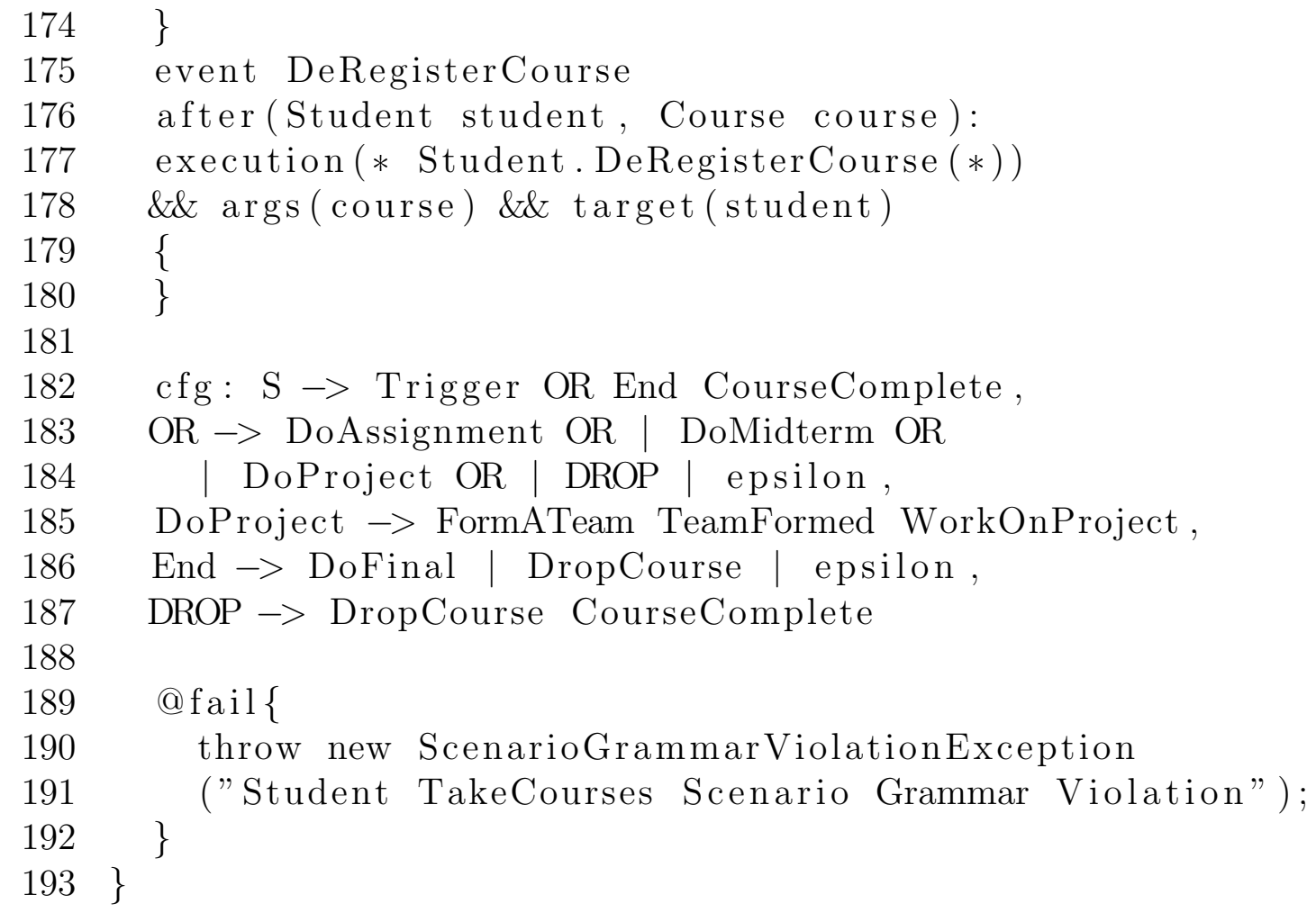

Much like the RegisterForCourses scenario, the above TakeCourses scenario has two monitor specifications associated with it. While, based on statements made above, one may assume there should be three specifications, two is enough to satisfy the intricacies of the above scenario. This is due to the fact that there are no events that take place outside of the atomic block but within the parallel block. As such, a single monitor specification can be used to handle both elements. While effective in this example, such an optimization would likely not be found in an actual implementation of these mappings. Three monitors would instead be generated.

The first monitor specification is the parent specification, containing all elements outside of the parallel block. This consists of the Trigger for the scenario as a whole, the Terminate for the scenario, and the logic necessary to initialize the sub-scenarios (handling the parallel and atomic sections) and keep track of their completion (lines 19-27). The triggering of the sub-scenarios consists of a for loop that iterates through the courses of the current student and initializes a sub-monitor for each one (lines 16 
and 17). This sub-monitor is parameterized such that it is specific to the student, course pair (line 50).

As the second monitor (line 49) is significantly more intricate than the first, a more detailed breakdown of its events will be provided. The first event (the Trigger event) is the same as those above (lines 60-71). It simply initializes the monitor and the monitor's variables for use throughout the monitor. The next two events correspond to the DoAssignment and DoMidterm responsibilities (lines 72-82 and 83-93 respectively). Both of these responsibilities have constraints associated with them and so, have counter variables and assertions in their corresponding events. These combinations of variables and assertions are what are used to ensure that the number of occurrences never exceeds the specified constraints. Also important to note is that any events that may follow a constrained event must include the checks to ensure that the prior event has met its constraints. This can be seen in the DoFinal event (lines 119-136). It contains the assertions to check that all previous events (those in the OR statement such as DoAssignment) have satisfied their constraints.

The next events are those resulting from including the grammar contained in the unbound DoProject responsibility. The first event corresponds to the FormATeam responsibility (lines 94-102) and is the event used to check the constraints of the event in the scenario. The following two events (lines 103-118) are simply the events corresponding to the next two responsibilities in the DoProject grammar.

All three of the above events (not including the first two of the DoProject unbound event) also include a firing statement to terminate the monitor (lines 81, 92, and 117). This is because a course may not have a final or a project and as such, may terminate after a midterm or an assignment as long as the constraints have been met. 
The final event before the alternative branch is the DoFinal event (lines 119-136). As mentioned previously, this event checks the constraints on the DoFinal responsibility as well as those that occur (or possibly occur) immediately before. Given that it may also be a final event in the grammar, it also fires the CourseComplete event to possibly terminate the scenario (line 135).

The next event, DropCourse (line 137-147), is the first, and only, event in the scenario's alternative branch. Since it is the first event, it is responsible for checking the condition of the alternative branch. Namely, that the LastDayToDrop event has not yet been fired. Since there are no constraints on this event and none to check from previous events, it simply fires the CourseComplete event.

All events corresponding to responsibilities have now been handled in the monitor. The remaining events are used to verify the scenario is complete and to include all possible responsibilities for checking the atomic property. The first of these is the CourseComplete event (lines 148-161). This event is only recognized when the monitor is in a valid state for completion. That is, the blackboard event (CourseComplete) that triggers this monitor event occurs every time a possible termination event occurs (as mentioned above), but the condition on this event prevents it from being recognized unless the monitor is in an appropriate state. For example, if there is no final, the firing of the CourseComplete event by the DoAssignment event may terminate the monitor as long as the constraints of all events in the OR statement have been satisfied. On the other hand, if there is a final, the CourseComplete event will be ignored unless it originates from the DoFinal event whose constraints must be satisfied.

Following the above event definitions is the CFG used to define the valid temporal ordering of event occurrences (lines 182-187). This CFG is particularly interesting because it illustrates how an alternative can be handled in the formalism of a monitor. This is done by including a non-terminal at each point in the CFG that the alternative 
branch may be taken. This non-terminal is a second path of execution that the scenario may take and does not join back with the original path. In the case above, this can be seen with the DROP non-terminal.

\subsubsection{Discussion of Difficulties}

The following subsection will present the difficulties that needed to be overcome and the major changes that were made during the development of the mapping from ACL to JavaMOP. This will be done through a discussion of the changes made between each iteration of the development and the reasoning behind each change. Over the course of the case study, the project went through nine iterations. These can be found online [61].

The first major change during the development (from iteration one to iteration two) was to properly handle the sub-scenarios described in the previous section. That is, to properly handle parallel and atomic blocks in a scenario. The original plan was to handle all aspects of a scenario in a single monitor specification. It was quickly realized however that this would not be possible due to how the parametric monitors recognize events and monitor properties. As such, the change was made to using multiple monitors for a single scenario where parent monitors would use blackboard events to trigger the initialization of sub-monitors.

The original attempt at handling the temporal aspects of ACL was to do so without the use of the logical formalisms in JavaMOP. Although originally chosen for its inclusion of grammars as a possible formalism, it was thought that the scenario semantics would be able to be better handled through the use of a custom tracking mechanism. This however ultimately turned out to be more work and less elegant than simply utilizing the CFG plugin of JavaMOP and so, the change to using CFGs was made. Iteration three therefore dropped the custom temporal tracking and implemented CFGs for each scenario monitor specification. 
Iteration four involved two major additions to the mapping. The first of these was the separation of the observabilities, contract variables, and invariants from the monitors and into their own contract classes (described previously). The second addition was the use of a separate monitor to do all of the non-temporal assertions (also described above).

Iteration five did not involve a huge change to the mapping, but did have a large impact on the functionality of the monitors. This iteration involved the switch to using JavaMOP creation events. Prior to this iteration, any event occurrence could trigger the creation of a new monitor instance if one did not already exist. This was causing many issues as some events had parameters not relevant to the monitors parametric trace. As a result, these events would still end up triggering the creation of new monitors, which ultimately resulted in grammar violations in monitors that shouldn't have still existed. The use of creation events enforced that only specified events could trigger the initialization of new monitor instances, eliminating the extra undesired monitors.

The next iteration, six, was conducted to properly handle atomic sections. It was originally thought that the logic formalism would inherently handle atomic sections in that only the events specified in the CFG would be allowed to execute and only in the order specified without triggering a CFG failure. It was realized however that this was not actually handling atomic sections properly in that any other events not defined in the atomic sections monitor, but in the original contract itself, could still occur without resulting in a failure. To account for this, all events in the contract but not in the atomic section needed to be added to the atomic monitors, but not be included in the monitor's CFG. This would ensure that if any event from the contract but not in the atomic section occurred, the atomic monitor would be able to detect its occurrence and identify the violation of the atomic property. This solution however presented a problem with how the atomic monitor was being created. The use of the 
constructor as a monitor initializer meant that as soon as a new instance of a class was created, the monitor would be created and thus start monitoring for the events. The problem was that atomic sections do not necessarily occur immediately after initialization of an object and as a result, the new method of including all events in the atomic monitor would cause a violation of the CFG if any event other than those included in the atomic section occurred, even outside of the atomic section (that is, if a non-atomic event occurs after initialization but before entering the atomic section). The discovery of this problem also led to the identification of another problem in how the atomic monitor was handling trace slicing. The lack of necessary parameters in the creation event was resulting in incorrect parametric slicing and the execution of some methods were not being recorded.

The solution to the above issue was to modify how the Blackboard class fired events, as well as how the atomic monitor was initialized. With respect to the Blackboard Fire method, it was overloaded such that it could be called with either an ACLEvent object as a parameter, or with the individual elements of an ACLEvent. The atomic monitor could then contain a Trigger event as a creation event (no longer using a constructor as a creation event) that would watch for the execution of the Fire() method where Fire() contained both a Student object and Course object (the parameters of the atomic monitor). This ensured that a monitor would be initialized for each instance of a \{Student, Course $\}$ pairing and thus correctly handle the parametric trace slicing for the atomic section. The use of this new approach (and its removal of the newInstance event) also allowed for the original implementation of the additional events in the atomic monitor to enforce the atomic property.

The changes made from iteration six to iteration seven ultimately had no impact on the functionality of the monitors, but were to improve performance and restore the monitors to how they were originally intended to be. During their initial development, a bug in the JavaMOP code was identified that was causing a stack overflow exception 
when generating monitors if the formalism contained too many events. This was found to be due to how JavaMOP calculates its enable sets (described in [59]). As a workaround, certain monitors needed to be broken into multiple monitors that had formalisms with few enough events. Upon reexamination at a later point it was found that a temporary fix could be implemented that would allow complete formalism specifications in a single monitor. This workaround was to modify the JavaMOP batch file to increase the Java stack size when generating monitors from the specifications. As a result, the previously broken up monitors were able to be combined back into single specifications.

Iteration eight implemented a new means of triggering scenario monitors. Instead of being created upon each new instance of an IUT contract object, they are instead created and started based on the trigger condition within the ACL. In the case of an event observation trigger, the main contract (i.e. 〈Type〉_Checks.mop) contains events and their respective handlers for each possible trigger event in that contracts scenarios. Upon occurrence of the triggering event (and any accompanying conditions), the event in the main contract monitor is triggered which in turn fires a custom blackboard event used to trigger the creation the appropriate scenario monitor. This is a more direct mapping from the ACL and more appropriately handles the creation of new scenario instances. For example, in the event of a second term starting, the TakeCourses scenario will properly be triggered a second time instead of needing some form of special loop to bypass the newInstance event on a second term. The new () trigger was left as is as it denotes that a scenario monitor instance should be created and started upon creation of a new IUT object.

The final iteration was to correct an error in how some atomic monitors functioned. In cases where an atomic section ends a scenario, there was no error. The issue came into effect when an atomic section occurs mid-scenario. This is due to how Java removes old objects. Since the garbage collector does not immediately remove old 
instances of objects, an atomic monitor may remain in existence after it has reached its termination event. The monitor would therefore still be actively monitoring for events. Since an atomic monitor includes all possible events from the contract, any event occurring after an atomic monitor's completion would trigger a violation from the atomic monitor. To avoid this issue, a flag variable was added to atomic monitors that would effectively disable the monitor upon completion and only re-activate the monitor upon occurrence of the triggering event. While not actually disabling the monitor, the variable forces the monitor to only recognize and record violations while set to true, indicating an active monitor. Otherwise, all violations of the atomic grammar are simply ignored.

\subsection{Verification Technique}

The method used to verify this feasibility was to include logging functionality in the JavaMOP monitors. After IUT execution, these logs (one log per monitor specification) contained the originating monitor, event ID, and event context for each event that occurred during execution (See Appendix B).

To execute the tests used for the verification of our mappings, a set of driver programs were written. These driver programs used the implementations developed for each case study to execute a sequence of method calls that would be either valid or invalid based on the ACL contracts. Each driver was implemented to test a single important of ACL (e.g. atomic sections). They were then used in a series of tests where the sequences of method calls varied to test different paths of execution through the IUT, based on the scenarios of the ACL contracts. As well as testing invalid sequences of calls, tests were also implemented that checked both valid and invalid parameter values in the method calls. After each driver was executed, the logs were

compared against the driver's method call sequence to verify they matched. An 
example of one of the test drivers used and the corresponding logs can be found in Appendix B.

In terms of coverage of ACL, we are confident that the combination of the two case studies (the university and yahtzee studies) is sufficient to support our selection of JavaMOP as a feasible framework to map to. The yahtzee case study provides much in the way of using ACL contract variables, while the university case study covers the intricacies that may be found in the various temporal elements of ACL scenarios.

\subsection{Conclusion}

As has been shown in the above case studies, ACL scenarios contain many intricacies that need to be handled when translating contracts into a runtime verification tool. They have also illustrated that JavaMOP provides the functionality necessary to be able to handle these intricacies. As such, it provides a feasible solution to properly monitor complex ACL semantics.

Once it was verified that JavaMOP could provide a feasible solution, an elementby-element mapping from ACL to JavaMOP was developed. This mapping will be presented in the next chapter. 


\section{Chapter 4}

\section{ACL to JavaMOP}

\subsection{Overview}

The following chapter provides a more detailed overview of our solution to the problem described in Chapter One and illustrated in Chapter Three. More specifically, it will provide an element-by-element mapping from ACL to JavaMOP. There will be no descriptions of the ACL elements as these can be found in [63]. As such, only the description of how each element may be implemented with JavaMOP will be provided. It must be emphasized that the verification of these mappings (beyond our case studies) is out of the scope of this thesis. More precisely, the implementation and verification of an ACL to JavaMOP generator is a separate thesis currently in progress.

Any elements not included in the following descriptions can be considered to have a direct mapping into JavaMOP. As such any description in the following would be trivial and unnecessary. 


\subsection{Elements Mapping}

\subsubsection{Using Declarations}

The current JavaMOP implementation does not include the ability to import other monitor specifications. However, through recent experimentation we have found that the newest implementation of JavaMOP (This is the version we are using. However, due to lack of documentation, this feature was discovered too late in development to implement.) provides the means to do so through the use of an include keyword in the monitor declaration. This will provide a means to map the using declaration into JavaMOP. This is left as future work.

\subsubsection{Contracts}

Contracts map to one or more JavaMOP monitor specifications. Each contract has a corresponding monitor specification to handle all precondition, postcondition, and invariant checks. It also has one or more specifications for each scenario contained within the contract. For example, a scenario that has a parallel block will have a monitor for the scenario as a whole, as well as a monitor that corresponds to the possible instances of the parallel block. To go along with the monitor specifications, each contract also has a corresponding Java class of the form 〈contract_class〉_Contract.java (EG: Student_Contract.java). This Java class contains all of the contract scoped variables (EG parameters), observabilities, and invariants.

JavaMOP does not support abstract monitors. These must be flattened into instance-specific monitor specifications.

Inheritance is also handled by flattening. JavaMOP (AspectJ) will capture polymorphic calls for all subtypes of a class. (For example, an event that takes a parameter of type Course will also capture occurrences of the method triggered by 
subclasses of type Course (i.e. ProjectCourse instances)). As well, the java contract class corresponding to the parent class must also be inherited by the java contract class corresponding to the subclass (e.g., the ProjectCourse_Contract.java class must extend the Course_Contract.java class).

\subsubsection{Bind Point Expression}

〈IUT type instance).bindpoint

The contract instance corresponding to the IUT type instance can be retrieved using the Blackboard. This is done upon creation of each new monitor instance so that the monitor can store this reference in a local variable. For future reference, this local variable can be used to access the contract class instance.

〈contract class instance).bindpoint

Upon creation, each contract class has a local variable that stores a reference to the IUT instance the contract is bound to. This variable is accessible from any monitor that has a local variable pointing to the contract class through the use of: <contract instance).instance.

\subsubsection{Context Access Expression}

A variable is included in each monitor called context that provides context access

functionality. This variable stores a reference to the instance of the IUT object that the monitor is bound to.

\subsubsection{Value Access Expression}

Accessing return values in JavaMOP is handled in the advice specification part of an event's definition. The returning() expression is used, with a parameter type 
and ID in the brackets, to access the value returned by the method the event will be bound to. The parameter type must match the variable type being returned. The parameter ID can then be used in the event handler to access the value returned by the method.

\subsubsection{Parameter Access Expression}

Since parameters are included in the contract classes, the contract class reference variables in the monitors must be used to access parameter values. This is done through the use of $\langle$ Reference Variable $\rangle$.〈Parameter ID $\rangle$.

\subsubsection{Don't Care Expression}

To implement the don't care expression, a Type is used in an event's args() list in place of the parameters defined in the event's advice spec. This specifies that instead of a specific value being required, it simply has to be of the specified type. For example, in place of event Test before(String s): execution(**.Test(*)) \&\& $\operatorname{args}(\mathrm{s})$ the event definition event Test before( $)$ : execution(**.Test $(*)) \& \&$ $\operatorname{args}($ String) would be used.

\subsubsection{Variables}

JavaMOP supports the same types of variables as ACL. The only consideration that needs to be taken into account is the scope of the variable in the ACL contracts. If it is a contract-scope variable, it is included in the corresponding JavaMOP contract class. If it is a variable from a responsibility (where its use spans two or more JavaMOP

events) or a scenario however, it is included in the JavaMOP monitor corresponding to the context from which it is taken. For example, a variable declared within a scenario is only included in that scenario's corresponding monitor. 


\subsubsection{Parameters}

Parameters are handled much like contract variables. They are included in the contract class that corresponds to the contract they are included in. The difference is that they are declared as final (and possibly static, depending on the parameter), and must be set prior to IUT execution. This pre-execution binding of values would be handled by the binding engine. However, as there is no implementation of a binding engine yet, the parameter values are instead specified upon initialization of the contract class.

\subsubsection{Structure}

JavaMOP does not currently support static checks. The JavaMOP group however claims that these will be added in a future release of the framework [60].

\subsubsection{Observability Methods}

\section{Bound Observability}

Bound observabilities are handled in JavaMOP in the contract class that corresponds to the contract the observability is from. They are defined as Java methods that use the contract class's instance variable to make a call to the necessary IUT method to retrieve the required information.

\section{Defined Observability}

Defined observabilities are also included in the contract class as Java methods. There is a direct mapping between the content of defined observabilities in ACL contracts

and in the corresponding contract classes. That is, the logic contained within a defined observability may be used within the corresponding Java method without any modification. 


\subsubsection{Invariants}

Like observabilities, invariants are included as methods in the contract classes. Each invariant method uses JUnit assertions to run the checks defined in the ACL contract they originate from. A single method is then also included in the contract class that makes a call to all invariant methods. This simplifies the use of the invariant methods in the monitors that need to make the checks. In place of calling all invariant methods in the before and after events, a single call to the invariant method (which in turn calls each individual invariant check) of the monitor's contract class may be used.

\subsubsection{Responsibilities}

\section{Bound Responsibilities}

These directly correspond to JavaMOP events. Depending on the purpose of the monitor the responsibility is mapped to, it may have one or two corresponding JavaMOP events. That is, if the monitor is a checks monitor (used for checking pre- and postconditions and invariants), and the responsibility has both pre- and post-conditions, then there will be two events corresponding to the single responsibility. Otherwise, a responsibility maps to a single JavaMOP event.

\section{Unbound Responsibilities}

As unbound responsibilities each contain a grammar of responsibilities, they do not map to any actual JavaMOP element. Instead, at each point where they are included in a scenario, the contained grammar is flattened into that scenario. That is, the grammar contained in the unbound responsibility is included in place of the responsibility in the scenario. 
Special Responsibilities

New

JavaMOP supports an event type (through AspectJ) that directly corresponds to the ACL new responsibility. This is the new() pointcut, which monitors the execution of class constructors. The rest of the mapping for this responsibility is the same as standard responsibilities.

Finalize

There is no event that corresponds to the ACL finalize responsibility. This is because this responsibility would normally map to some form of destructor method. As Java uses a garbage collector, it does not support any form of destructor and, as such, there is no construct for this ACL element to map to.

\subsubsection{Responsibility Bodies}

\section{Contract Instance Declaration}

The JavaMOP mapping handles this much the same way as described in the ACL algorithms [63]. However, lists of contract instances maintained in the Blackboard class are used to retrieve the contract instances.

In the case of a scalar type variable (from page 124 of the specification document [63]):

1. If no contract instance of the requested type exists, execution will terminate with an exception.

2. Will use the context of the scenarios firing event. That is, the event will retrieve the contract class instance bound to the monitor from which the scenario was triggered. (combines step 2 and 3 of the ACL algorithm).

3. If the above steps are not sufficient to retrieve the desired contract instance, then the user must be prompted for the instance. This is not currently implemented 
as it is a responsibility of the binding engine.

In the case of a list type:

Essentially the same as the ACL algorithm above but will simply return the list of contracts maintained by the Blackboard for the specified contract type.

\section{Belief Statement}

The mapping for a belief statement is accomplished by converting the checks contained in the belief into JUnit assertions (namely, assertTrue()).

Pre Statement

Just as the checks in a belief statement, a pre statement is mapped directly to a JUnit assertion.

\section{PreSet Statement}

There is no special element in the JavaMOP mapping to handle this type of statement. It is simply mapped to a variable assignment located in the before event that corresponds to the responsibility the statement is located in.

\section{Post Statement}

Handled the same as pre statements, post statements are implemented as JUnit assertions.

Fire Statement

This is handled using the Blackboard class. A call to the blackboard's Fire(ACLEvent e) is used to fire a global event that all monitors may then observe. The Fire() method is also overridden to accept the individual data members 
of an ACLEvent as parameters. This allows for their inclusion as parameters in event definitions. More specifically, it is overridden to accept a) only an ID parameter, b) an ID and a context parameter, or c) an ID, context, and data object parameter (passed by value). The data object may only be included as a single object currently. If more than a single object is necessary to be able to include all pertinent data, a list of objects could be included instead and still be accepted as a single parameter.

\section{Execute Statement}

This would correspond to the proceed keyword used in AspectJ around advice. As this type of advice is not usable in JavaMOP, the execute statement is only used to define where the split occurs for before and after advice events.

\subsubsection{Stub Responsibilities}

In the JavaMOP mapping, stub responsibilities are flattened into any grammar where they are referenced. Any variables declared within the stub also need to be defined as local variables to any monitors that correspond to a contract instance the stub is used in.

\subsubsection{Scenarios}

A scenario corresponds to a new monitor specification with an associated logical formalism (CFG). The events in the monitor correspond to the ACL events and responsibilities that may occur in the scenario, and the logical formalism corresponds to the scenario grammar.

The strict modifier maps to an atomic scenario, following the same description as defined in the section on atomic elements (upcoming). 


\section{Trigger Statement}

The trigger statement has two forms that are used in JavaMOP. To handle an ACL scenario triggering on a new instance of an object, the mapping is simply an event in the scenario monitor that catches a constructor execution. This event is then the first event that must occur in the scenario monitors logical formalism. The second means of handling the trigger accounts for a responsibility occurrence or an observation of an event. Both are first caught in the contract's main monitor (the monitor containing all pre and post checks) which then in turn fires events to start instances of the appropriate monitors. This is handled this way to ensure proper parametric trace slicing.

All events corresponding to trigger statements are declared as creation events in JavaMOP so that only those events may create new instances of the monitor.

\section{Terminate Statement}

To handle the terminate statement, an event is included in each scenario monitor that is triggered every time a different event occurs that may end the scenario. This termination event contains as a condition in its definition the necessary checks to ensure it will only be recognized if the monitor is in a valid state for termination. That is, as long as all constraints in the scenario have been satisfied. The termination event is then included as the final event in the monitor's logical formalism. This will ensure a formalism match will only occur if the terminate event is recognized. In the case of an empty terminate statement, the final event is omitted and the formalism will match upon handling the final event in the grammar. 


\section{Atomic Temporal Element}

To be properly handled in JavaMOP, atomic sections of a scenario need to be defined as separate monitors. These separate monitors include all possible events from the parent contract while having a logical formalism containing only those responsibilities that occur in the contract's atomic section. This enforces that only the events within

the atomic block can occur and only in the specified sequence until the atomic block is complete. Also included in the atomic monitors is a flag variable. As a monitor may remain in existence until collected by the garbage collector, it may still recognize events after it has finished. This would be detected as a violation of the grammar. The monitor is therefore only active while the flag is set to true, and will ignore any violations if it is set to false. It is set to true upon the atomic sections triggering event occurring, and false upon completion.

\section{Parallel Temporal Element}

In the mapping to JavaMOP, parallel blocks translate to their own monitors. These monitors are created and triggered by the parent scenario monitor using a specialized form of the Blackboard.Fire() method as discussed previously in the section on the trigger statement.

\section{Alternative Element}

The current mapping to JavaMOP supports the alternative element as a branch in the monitors' formalisms (CFGs). The location of the branch in the main path through the formalism needs to be included as a nonterminal at each spot the branch may occur. That is, in the main path through the grammar, the alternative nonterminal is included after each event as the alternative may occur at any time. This can be seen in the CFG of the Student_Scenario_TakeCourses_sub monitor specification in 
the university case study discussed in Chapter Three.

\section{New Instance Element}

The new instance element maps to an AspectJ new event in JavaMOP. This event is the same as those corresponding to the new responsibility. The new IUT instance corresponding to the new instance element can then be assigned to a monitor variable from within the event handler.

\section{Observe Element}

The observe element is mapped directly into a JavaMOP event. The difference between an observe event and a responsibility event is that the observe event will always watch for the occurrence of the Blackboard's Fire method. Since all observe events monitor the same method, the appropriate conditions must be included in the event definition to ensure that the observation only occurs for the proper fired event. That is, the condition must specify the exact event ID as well as the appropriate context and data if necessary.

One or More (+) Repetition Operator

These can be used directly. They are placed on events in a monitor's formalism.

\section{Zero or more $\left(^{*}\right)$ Repetition Operator}

The same as the previous operator, these can be used directly. They are placed on events in a monitor's formalism.

\section{Optional (?) Repetition Operator}

In place of using an operator in the formalism, the mapping of the optional repetition operator is accomplished through the use of an OR condition in a monitor's property. 
In place of the single event, an OR condition is included in brackets stating that either the event or epsilon (representation of the empty string) may occur.

Fixed ([n]) Repetition Operator

This type of operator is handled with assertions. These assertions are included in all events that may follow the event with the constraint. The assertion checks that the number of times the previous event has occurred matches the value of the repetition operator. For example, in the student monitor, the DoFinal event has the check for the constraint on the DoAssignment event. This check will ensure that for the DoFinal event to occur without failure, the constraint on the DoAssignment event must be satisfied.

Ranged ([p to q]) Repetition Operator

These are handled the same way as the fixed repetition operator. An assertion is used in each event's following event to verify that its predecessor has satisfied the repetition constraint.

\subsection{Summary of Mappings}

The following table will provide a list of all ACL elements considered. It will categorize the mapping of each element as trivial, ignored, or done. As well, for those elements where a mapping was implemented in the previously discussed case studies, a confidence level will be given. This confidence level will be one of three possibilities: very confident, confident, and not confident.

Important to note is the exclusion of interactions from this feasibility study. This is because they are currently under investigation as to whether they will still be necessary with the reimplementation of the Validation Framework. To speculate on 
this is outside of the context of this thesis and is therefore marked as ignored in the following summary.

\begin{tabular}{|c|c|c|}
\hline Element & Mapping & Level of Confidence \\
\hline Using Declarations & Done & Not Confident \\
\hline Contracts & Done & Very Confident \\
\hline Namespaces & Ignored & - \\
\hline Contract Declarations & Trivial & Confident \\
\hline Contract Bodies & Done & Very Confident \\
\hline Invocation Expression & Trivial & Very Confident \\
\hline Bind Point Expression & Done & Very Confident \\
\hline Context Access Expression & Done & Very Confident \\
\hline Value Access Expression & Done & Very Confident \\
\hline Counter Access Expression & Trivial & Very Confident \\
\hline Iterator Access Expression & Trivial & Very Confident \\
\hline Parameter Access Expression & Done & Very Confident \\
\hline Don't Care Expression & Done & Confident \\
\hline Variables & Done & Very Confident \\
\hline Parameters & Done & Very Confident \\
\hline Structure & Ignored & - \\
\hline
\end{tabular}




\begin{tabular}{|c|c|c|}
\hline Observability Methods & Done & Very Confident \\
\hline Bound Observabilities & Done & Very Confident \\
\hline Defined Observabilities & Done & Very Confident \\
\hline Invariants & Done & Very Confident \\
\hline Responsibilities & Done & Very Confident \\
\hline The New Responsibility & Done & Very Confident \\
\hline The Finalize Responsibility & Ignored & - \\
\hline Bound Responsibilities & Done & Very Confident \\
\hline Unbound Responsibilities & Done & Very Confident \\
\hline Responsibility Bodies & - & - \\
\hline Contract Instance Declaration & Done & Confident \\
\hline Belief Statement & Ignored & - \\
\hline Choice Statement & Trivial & Very Confident \\
\hline Each Statement & Trivial & Very Confident \\
\hline Loop Statement & Trivial & Very Confident \\
\hline Pre Statement & Done & Very Confident \\
\hline PreSet Statement & Done & Confident \\
\hline Post Statement & Done & Very Confident \\
\hline
\end{tabular}




\begin{tabular}{|c|c|c|}
\hline Fire Statement & Done & Very Confident \\
\hline Execute Statement & Ignored & - \\
\hline Stub Responsibilities & Done & Confident \\
\hline Scenarios & Done & Confident \\
\hline Trigger Statement & Done & Very Confident \\
\hline Terminate Statement & Done & Confident \\
\hline Atomic Temporal Element & Done & Confident \\
\hline Parallel Temporal Element & Done & Confident \\
\hline Alternative Element & Done & Confident \\
\hline New Instance Element & Done & Confident \\
\hline Observe Element & Done & Very Confident \\
\hline One or More $(+)$ Repetition Operator & Done & Very Confident \\
\hline Zero or More (*) Repetition Operator & Done & Very Confident \\
\hline Optional (?) Repetition Operator & Done & Very Confident \\
\hline Fixed ([n]) Repetition Operator & Done & Very Confident \\
\hline Ranged ([p to q]) Repetition Operator & Done & Confident \\
\hline Metric Methods & Ignored & - \\
\hline Reports & Ignored & - \\
\hline
\end{tabular}




\begin{tabular}{|l|c|c|}
\hline Exports & Ignored & - \\
\hline Interactions & Ignored & - \\
\hline
\end{tabular}

Table 2: Categorized listing of considered ACL elements. 


\section{Chapter 5}

\section{Conclusion and Future Work}

\subsection{Summary of Contribution}

Our work in this thesis illustrates the feasibility of mapping the ACL specification language to the JavaMOP framework. We have implemented this mapping for two different case studies, illustrating how the various ACL elements and semantics can be mapped to JavaMOP.

The first of these case studies, that of the Yahtzee game, provides a detailed overview how JavaMOP can support the contract variables of ACL. These variables are dynamic (can be updated throughout the execution of an IUT) and can be used as test oracles during execution.

The second case study, the university system, illustrates the mappings from some of the more complex and intricate elements and semantics of ACL into JavaMOP. This primarily consists of ACL scenario grammars and the various temporal elements they support. More specifically, atomic elements, parallel elements, and alternatives, and the various combinations of these.

Ultimately, we have shown that ACL contract specifications can be supported for runtime verification with the JavaMOP framework. Each possible contract instance from the ACL/VF tool has a corresponding monitor (or set of monitors) equivalent in 
JavaMOP. These monitors include variables that can be used as dynamic test oracles for runtime checks, as well as support logical formalisms in the form of context-free grammars to verify temporal ordering of events.

We have also added our own subsystem to the JavaMOP framework to support the firing and observing of global events, that is, events visible to all contract instances. We have called this system the Blackboard. Any JavaMOP monitor may fire an event to the Blackboard, or observe the Blackboard for a specific event occurrence. We have also added to ACL events in the form of a context object and a data object. As such, events are now parameterized (as has been shown in the case studies in Chapter Three).

Also managed by the Blackboard subsystem are contract class instances. These class instances correspond to contract instances and contain elements such as contract variables, observabilities, and invariants. As such, all monitors related to a single contract instance (as well as the contract's scenario instances) need to contain a reference to the corresponding contract class instance. Since each contract instance corresponds to an instance of an IUT type, the Blackboard keeps track of contract classes through a mapping from an IUT instance ID to its corresponding contract class instance. Monitors may then use their local reference to the instance they are bound to to access their corresponding contract class.

\section{$5.2 \quad$ Future Work}

As we have shown the feasibility of a mapping from ACL to JavaMOP, we can briefly present additional features and refinements that can be made to further improve this mapping. These will be discussed briefly.

In addition to the features that will follow, there are a number of separate theses 
examining modifications to ACL. This includes modifications such as the parameterized events introduced in this thesis, and generative version of ACL to specify design patterns. There is also work being done on an implementation of a binding engine (mentioned briefly in this thesis), and an implementation of a tool that will implement the mappings introduced in this thesis.

\subsubsection{Custom ACL Scenario Formalism}

The above mapping uses the CFG plugin of JavaMOP to support the ACL scenario

grammar. As there is not a direct mapping between these two grammars, a number of workarounds had to be implemented to properly support the ACL semantics. These are not ideal solutions.

As JavaMOP provides the extensibility to write custom property plugins, a recommended feature to be added is an ACL scenario formalism. That is, a plugin written specifically to support the syntax and semantics of ACL. This would provide a means of specifying the additional repetition operators discussed previously, as well as specific temporal elements such as atomic, parallel, and alternative sections.

\subsubsection{JavaMOP Abstract Monitors}

Late experimentation with the new version of JavaMOP revealed that it included support of abstract monitors. As these were not supported in our work, they have been left as a future refinement of the mapping. Use of actual abstract monitors would provide a more direct mapping between ACL contracts and JavaMOP monitors.

\subsubsection{JavaMOP Inheritance}

Also discovered late in the experimentation was JavaMOP's support of monitor inheritance. Left as future work, use of this feature would eliminate the need for some 
of the workarounds present in the previous case studies to properly handle inherited contracts (e.g. the Course and ProjectCourse contracts of the university case study).

\subsection{Conclusion}

As described by Grieskamp [12], scenario-based approaches to model-based testing are generally preferred over state-based approaches. Not only do they provide an alternative that avoids issues inherent in state-based approaches (such as state explosion), but they also provide a specification closer to natural language. As such, Arnold et al. [23] argue for the adoption of scenario-based approaches, and more specifically, of a tool such as ACL/VF.

Issues with the existing .NET3.5 version of ACL/VF however prevent it from currently being a viable candidate for model-based testing. As a solution to this problem, we propose reimplementing ACL/VF through a mapping to an already developed tool, namely JavaMOP. In this thesis, we have successfully accomplished this mapping for two different case studies where the ACL contracts were developed independent of our work. As such, we argue that this reimplementation of ACL/VF through a mapping to JavaMOP is indeed a feasible solution. 


\section{References}

[1] P. Bourque and R.E. Fairley, eds., Guide to the Software Engineering Body of Knowledge, Version 3.0. www.swebok.org: IEEE Computer Society, 2014.

[2] J. Ryser and M. Glinz, "Scent: A method employing scenarios to systematically derive testcases for system test," tech. rep., 2000.

[3] A. Bansal, "A Comparative Study of Software Testing Techniques," vol. 3, no. 6, pp. 579-584, 2014.

[4] J. Corriveau and W. Shi, "On acceptance testing," International conference on software engineering research and practice (SERP 2013), Las Vegas, pp. 22-25, 2013.

[5] L. Copeland, A Practitioner's Guide to Software Test Design. Norwood, MA, USA: Artech House, Inc., 2003.

[6] J. Dooley, "Unit testing," in Software Development and Professional Practice, pp. 193-208, Apress, 2011.

[7] "JUnit." http://www.junit.org/. (Accessed: 2014-07-24).

[8] L. Briand and Y. Labiche, "A UML-based approach to system testing," Software and Systems Modeling, no. June, pp. 1-57, 2002.

[9] R. Miller and C. T. Collins, "Acceptance testing," Proc. XPUniverse, 2001.

[10] D. Arnold, J. Corriveau, and W. Shi, "Modeling and Validating Requirements Using Executable Contracts and Scenarios," in Software Engineering Research, Management and Applications (SERA), 2010 Eighth ACIS International Conference on, pp. 311-320, May 2010. 
[11] A. C. Dias Neto, R. Subramanyan, M. Vieira, and G. H. Travassos, "A survey on model-based testing approaches: A systematic review," in Proceedings of the 1st ACM International Workshop on Empirical Assessment of Software Engineering Languages and Technologies: Held in Conjunction with the 22Nd IEEE/ACM International Conference on Automated Software Engineering (ASE) 200\%, WEASELTech '07, (New York, NY, USA), pp. 31-36, ACM, 2007.

[12] W. Grieskamp, "Multi-paradigmatic model-based testing.," in FATES/RV (K. Havelund, M. Nez, G. Rosu, and B. Wolff, eds.), vol. 4262 of Lecture Notes in Computer Science, pp. 1-19, Springer, 2006.

[13] D. Arnold, An Open Framework for the Specfication and Execution of a Testable Requirements Model. PhD thesis, Carleton University, 2009.

[14] C. Nebut, F. Fleurey, Y. Le Traon, and J.-M. Jezequel, "Automatic test generation: a use case driven approach," IEEE Transactions on Software Engineering, vol. 32, pp. 140-155, Mar. 2006.

[15] D. Arnold, "The University Contract Evaluation Framework Walk Through," 2008.

[16] D. Jin and P. Meredith, "JavaMOP: Efficient parametric runtime monitoring framework," 2012 34th International Conference on Software Engineering (ICSE), pp. 1427-1430, 2012.

[17] J. Hunt, "Gang of four design patterns," in Scala Design Patterns, pp. 135-136, Springer International Publishing, 2013.

[18] R. V. Binder, Testing Object-oriented Systems: Models, Patterns, and Tools. Boston, MA, USA: Addison-Wesley Longman Publishing Co., Inc., 1999.

[19] V. Stolz and E. Bodden, "Temporal assertions using aspectj," Electron. Notes Theor. Comput. Sci., vol. 144, pp. 109-124, May 2006.

[20] M. D'Amorim and K. Havelund, "Jeagle: a JAVA Runtime Verification tool," pp. 1-20, 2004.

[21] D. Drusinsky, "The temporal rover and the atg rover," pp. 323-330, 2000.

[22] D. Arnold, J. Corriveau, and W. Shi, "Scenario-based validation: Beyond the user requirements notation," Software Engineering Conference (ASWEC), 2010. 
[23] D. Arnold, J. Corriveau, and W. Shi, "Reconciling Offshore Outsourcing with Model Based Testing," Software Engineering Approaches for Offshore and Outsourced Development, 2010.

[24] G. Leavens, A. Baker, and C. Ruby, "Jml: A notation for detailed design," in Behavioral Specifications of Businesses and Systems (H. Kilov, B. Rumpe, and I. Simmonds, eds.), vol. 523 of The Springer International Series in Engineering and Computer Science, pp. 175-188, Springer US, 1999.

[25] "IEEE Standard for System and Software Verification and Validation," IEEE Std 1012-2012 (Revision of IEEE Std 1012-2004), pp. 1-223, May 2012.

[26] M. Leucker, "Teaching runtime verification," Runtime Verification, pp. 34-48, 2012.

[27] N. Delgado, S. Member, A. Q. Gates, and I. C. Society, "A Taxonomy and Catalog of Runtime Software-Fault Monitoring Tools," vol. 30, no. 12, pp. 859$872,2004$.

[28] C. Ghezzi and S. Guinea, "Run-time monitoring in service-oriented architectures," Test and analysis of web services, 2007.

[29] L. Xuandong, W. Linzhang, and Q. Xiaokang, "Runtime verification of Java programs for scenario-based specifications," Ada-Europe 2006, no. 60425204, pp. 94$105,2006$.

[30] Y. Zhao and F. Rammig, "Online Model Checking for Dependable Real-Time Systems," 2012 IEEE 15th International Symposium on Object/Component/Service-Oriented Real-Time Distributed Computing, pp. 154-161, Apr. 2012.

[31] P. Arcaini, A. Gargantini, and E. Riccobene, "Components monitoring through formal specifications," Proceedings of the 17th international doctoral symposium on Components and Architecture, pp. 25-30, 2012.

[32] S. Sankar and M. Mandal, "Concurrent runtime monitoring of formally specified programs," Computer, vol. 26, pp. 32-41, Mar. 1993.

[33] D. Luckham, S. Sankar, and S. Takahashi, "Two-dimensional pinpointing: Debugging with formal specifications," Software, IEEE, no. January, 1991.

[34] D. Rosenblum, "A practical approach to programming with assertions," Software Engineering, IEEE Transactions on, vol. 21, no. 1, 1995. 
[35] A. Beguelin and B. Bruegge, "A configurable monitoring system for parallel programming," Proceedings of 2nd International Workshop on Configurable Distributed Systems, no. June 1993, p. 15213, 1994.

[36] "Dynamics: Comprehensive support for run-time monitoring," pp. 61-77, 2001.

[37] Z. Zhou, L. Wang, Z. Cui, X. Chen, and J. Zhao, "Jasmine: A Tool for ModelDriven Runtime Verification with UML Behavioral Models," 2008 11th IEEE High Assurance Systems Engineering Symposium, pp. 487-490, Dec. 2008.

[38] X. Li, X. Qiu, L. Wang, B. Lei, and W. E. Wong, "UML state machine diagram driven runtime verification of Java programs for message interaction consistency," Proceedings of the 2008 ACM symposium on Applied computing - SAC '08, p. 384, 2008.

[39] H. Barringer, A. Groce, K. Havelund, and M. Smith, "An Entry Point for Formal Methods: Specification and Analysis of Event Logs," Electronic Proceedings in Theoretical Computer Science, vol. 20, pp. 16-21, Mar. 2010.

[40] Y. Liao and D. Cohen, "A specificational approach to high level program monitoring and measuring," IEEE Transactions on Software Engineering, vol. 18, no. 11, pp. 969-978, 1992.

[41] S. Chodrow and M. Gouda, "Implementation of the sentry system," Software: Practice and Experience, pp. 1-20, 1995.

[42] G. Holzmann, "The model checker SPIN," IEEE Transactions on Software Engineering, vol. 23, pp. 279-295, May 1997.

[43] M. C. B. Alves, D. Drusinsky, and M.-T. Shing, "A Practical Formal Approach for Requirements Validation and Verification of Dependable Systems," 2011 Fifth Latin-American Symposium on Dependable Computing Workshops, pp. 47-51, Apr. 2011.

[44] D. Drusinsky and M.-T. Shing, "Verifying Distributed Protocols using MSCAssertions, Run-time Monitoring, and Automatic Test Generation," 18th IEEE/IFIP International Workshop on Rapid System Prototyping (RSP '07), pp. 82-88, May 2007.

[45] M. Karaorman and P. Abercrombie, "jcontractor: Introducing design-bycontract to java using reflective bytecode instrumentation," Formal Methods in System Design, pp. 1-44, 2005. 
[46] A. Mok, "Efficient Run-time Monitoring Of Timing Constraints," Proceedings Third IEEE Real-Time Technology and Applications Symposium, pp. 252-262.

[47] C. Colombo, G. J. Pace, and G. Schneider, "LARVA — Safer Monitoring of RealTime Java Programs (Tool Paper)," 2009 Seventh IEEE International Conference on Software Engineering and Formal Methods, no. i, pp. 33-37, 2009.

[48] I. Krüger, M. Meisinger, and M. Menarini, "Runtime verification of interactions: from MSCs to aspects," Runtime Verification, pp. 63-74, 2007.

[49] J. Rieken, "Design by contract for java-revised," Master's thesis, Department für Informatik, 2007.

[50] Y. Cheon and G. Leavens, "A runtime assertion checker for the Java Modeling Language (JML)," no. March, 2002.

[51] H. Barringer and K. Havelund, TraceContract: A Scala DSL for trace analysis. 2011.

[52] C. Allan and P. Avgustinov, "Adding trace matching with free variables to AspectJ," Proceedings of the 20th annual ACM SIGPLAN conference on Object oriented programming systems languages and applications, 2005.

[53] H. Barringer, D. Rydeheard, and K. Havelund, "Rule Systems for Run-time Monitoring: from EAGLE to RULER," Journal of Logic and Computation, vol. 20, pp. 675-706, Nov. 2008.

[54] M. Kim, M. Viswanathan, and S. Kannan, "Java-MaC: A run-time assurance approach for Java programs," Formal methods in System Design, vol. 24, no. 2, pp. 129-155, 2004.

[55] P. Meredith, D. Jin, F. Chen, and G. Rosu, "Efficient monitoring of parametric context-free patterns," pp. 148-157, Sept 2008.

[56] F. Chen and G. Rosu, "Mop: an efficient and generic runtime verification framework," ACM SIGPLAN Notices, pp. 569-588, 2007.

[57] K. Havelund and G. Rosu, "An Overview of the Runtime Verification Tool Java PathExplorer," Formal Methods in System Design, vol. 24, pp. 189-215, Mar. 2004.

[58] B. D’Angelo, S. Sankaranarayanan, C. Sanchez, W. Robinson, B. Finkbeiner, H. Sipma, S. Mehrotra, and Z. Manna, "Lola: runtime monitoring of synchronous systems," pp. 166-174, June 2005. 
[59] F. Chen, P. O. Meredith, D. Jin, and G. Rosu, "Efficient Formalism-Independent Monitoring of Parametric Properties," 2009 IEEE/ACM International Conference on Automated Software Engineering, pp. 383-394, Nov. 2009.

[60] P. Meredith, D. Jin, D. Griffith, F. Chen, and G. Rosu, "An overview of the mop runtime verification framework," International Journal on Software Tools for Technology Transfer, vol. 14, no. 3, pp. 249-289, 2012.

[61] J. Beltramin. www.scs.carleton.ca/ jeanpier/JoshuaBeltraminFiles.

[62] D. Arnold, "ACL/VF." vf.davearnold.ca. (Accessed: 2014-08-02).

[63] D. Arnold, "Another Contract Language 3.2 Specification Document," 2010. 


\section{Appendix A}

\section{Contract Class Example}

\section{A.1 Course Contract Class}

1 public class Course_Contract \{

2 Scanner reader $=$ new Scanner(System.in);

3 //Binding to iut class

4 public Course instance;

$5 \quad / /$ Contract variables

6 public static final boolean EnforcePreRequisites;

$7 \quad$ static \{

8 Scanner reader $=$ new Scanner (System .in);

9 System.out.println("Enter value binding

10 for EnforePreRequisites (true or false).");

11 EnforcePreRequisites = reader.nextBoolean ();

$12\}$

13 public final int NumMidterms;

14 public final int NumAssignments;

15 public final boolean HasFinal; 


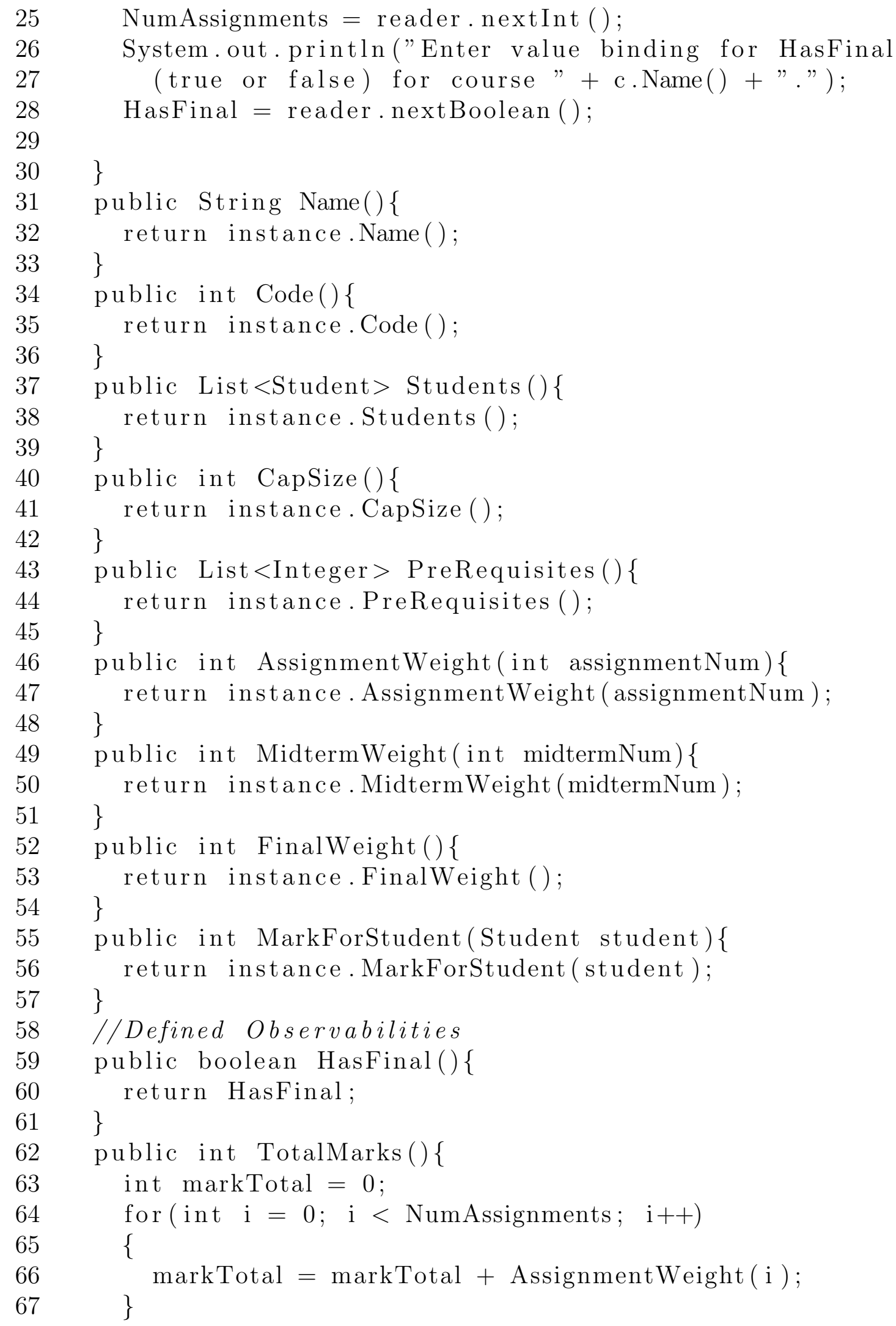




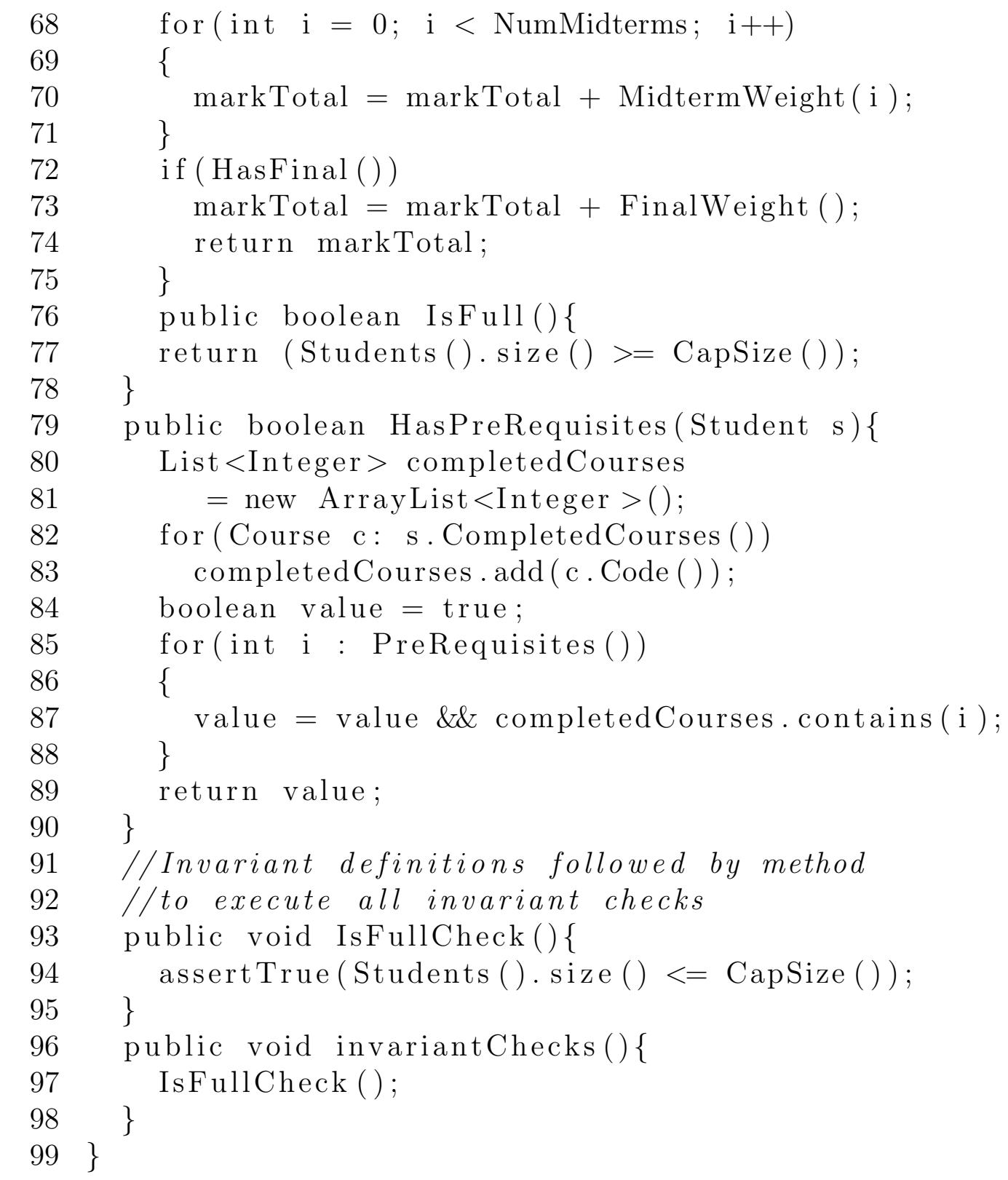




\section{Appendix B}

\section{Log Examples}

The following appendix provides an example of the logs used to check the correctness of the monitors generated from the ACL specifications. It begins with the logs themselves followed by the driver program used to drive the testing. This program contains a valid sequence of method calls that, when monitored, pass all of the ACL checks. During execution, the logs are generated based on the events detected and recorded by the monitors. The logs may then be compared against the driver program to ensure the sequence of method calls in the program match the event sequences in the logs. As well, the logs are specific to each monitor and as such may be also compared against the ACL specifications to verify that the execution was indeed valid.

The logs are quite basic in what they contain. They specify the monitor from which the recorded event originated, the name of the event, the type of check of the event (i.e. whether the event handled pre- or post-conditions), and the context of the event (i.e. the IUT instance to which the event's monitor was bound). 


\section{B.1 Course Checks Log}

\begin{tabular}{|l|l|l|l|}
\hline Monitor & Event Name & Check Type & Context \\
\hline Course Checks & newInstance & null & Course@e20699a \\
Course Checks & AddStudentNoPreReqCheck & Preconditions & Course@1720d359 \\
Course Checks & AddStudentNoPreReqCheck & Postconditions & Course@1720d359 \\
Course Checks & AddStudentNoPreReqCheck & Preconditions & Course@51cf1d17 \\
Course Checks & AddStudentNoPreReqCheck & Postconditions & Course@51cf1d17 \\
Course Checks & AddStudentNoPreReqCheck & Preconditions & Course@3c8e683a \\
Course Checks & AddStudentNoPreReqCheck & Postconditions & Course@3c8e683a \\
Course Checks & AddStudentNoPreReqCheck & Preconditions & Course@295cd6e5 \\
Course Checks & AddStudentNoPreReqCheck & Postconditions & Course@295cd6e5 \\
Course Checks & AddStudentNoPreReqCheck & Preconditions & Course@51cf1d17 \\
Course Checks & AddStudentNoPreReqCheck & Postconditions & Course@51cf1d17 \\
Course Checks & AddStudentNoPreReqCheck & Preconditions & Course@295cd6e5 \\
Course Checks & AddStudentNoPreReqCheck & Postconditions & Course@295cd6e5 \\
Course Checks & AddStudentNoPreReqCheck & Postconditions & Course@e20699a \\
\hline
\end{tabular}

\section{B.2 Course Scenario ReportMarks Log}




\begin{tabular}{|l|l|l|}
\hline Monitor & Event Name & Context \\
\hline Course Scenario ReportMarks & Trigger & Course@e20699a \\
Course Scenario ReportMarks & ReportMarks & Course@1720d359 \\
Course Scenario ReportMarks & Terminate & Course@1720d359 \\
Course Scenario ReportMarks & ReportMarks & Course@51cf1d17 \\
Course Scenario ReportMarks & ReportMarks & Course@3c8e683a \\
Course Scenario ReportMarks & Terminate & Course@3c8e683a \\
Course Scenario ReportMarks & ReportMarks & Course@295cd6e5 \\
Course Scenario ReportMarks & ReportMarks & Course@51cf1d17 \\
Course Scenario ReportMarks & ReportMarks & Course@e20699a \\
Course Scenario ReportMarks & ReportMarks & Course@295cd6e5 \\
\hline Courio ReportMarks & Terminate & Course@295cd6e5 \\
\hline Course & Terminate & Course $51 \mathrm{~d} 17$ \\
\hline
\end{tabular}

\section{B.3 Student Checks Log}

\begin{tabular}{|l|l|l|l|}
\hline Monitor & Event Name & Check Type & Context \\
\hline Student Checks & newInstance & null & Student@671ef55c \\
Student Checks & Selectcourse & PostConditions & Student@ebe5687 \\
\hline
\end{tabular}




\begin{tabular}{|c|c|c|c|}
\hline Monitor & Event Name & Check Type & Context \\
\hline Student Checks & RegisterCourse & PreConditions & Student@ebe5687 \\
\hline Student Checks & RegisterCourse & PostConditions & Student@ebe5687 \\
\hline Student Checks & Selectcourse & PostConditions & Student@ebe5687 \\
\hline Student Checks & RegisterCourse & PreConditions & Student@ebe5687 \\
\hline Student Checks & RegisterCourse & PostConditions & Student@ebe5687 \\
\hline Student Checks & Selectcourse & PostConditions & Student@ebe5687 \\
\hline Student Checks & RegisterCourse & PreConditions & Student@ebe5687 \\
\hline Student Checks & RegisterCourse & PostConditions & Student@ebe5687 \\
\hline Student Checks & Selectcourse & PostConditions & Student@ebe5687 \\
\hline Student Checks & RegisterCourse & PreConditions & Student@ebe5687 \\
\hline Student Checks & RegisterCourse & PostConditions & Student@ebe5687 \\
\hline Student Checks & Selectcourse & PostConditions & Student@479482ea \\
\hline Student Checks & RegisterCourse & PreConditions & Student@479482ea \\
\hline Student Checks & RegisterCourse & PostConditions & Student@479482ea \\
\hline Student Checks & Selectcourse & PostConditions & Student@479482ea \\
\hline Student Checks & RegisterCourse & PreConditions & Student@479482ea \\
\hline Student Checks & RegisterCourse & PostConditions & Student@479482ea \\
\hline Student Checks & Selectcourse & PostConditions & Student@671ef55c \\
\hline
\end{tabular}




\begin{tabular}{|l|l|l|l|}
\hline Monitor & Event Name & Check Type & Context \\
\hline Student Checks & RegisterCourse & PreConditions & Student@671ef55c \\
Student Checks & RegisterCourse & PostConditions & Student@671ef55c \\
Student Checks & DoAssignment & PreConditions & Student@479482ea \\
Student Checks & DoAssignment & PreConditions & Student@ebe5687 \\
Student Checks & DoAssignment & PreConditions & Student@ebe5687 \\
Student Checks & DoMidterm & PreConditions & Student@ebe5687 \\
Student Checks & DoAssignment & PreConditions & Student@ebe5687 \\
Student Checks & DoMidterm & PreConditions & Student@ebe5687 \\
Student Checks & DoAssignment & PreConditions & Student@ebe5687 \\
Student Checks & DoMidterm & PreConditions & Student@ebe5687 \\
Student Checks & DoAssignment & PreConditions & Student@ebe5687 \\
Student Checks & DoAssignment & PreConditions & Student@ebe5687 \\
Student Checks & DoMidterm & PreConditions & Student@ebe5687 \\
Student Checks & DoAssignment & PreConditions & Student@ebe5687 \\
Student Checks & DoMidterm & PreConditions & Student@ebe5687 \\
\hline
\end{tabular}




\begin{tabular}{|l|l|l|l|}
\hline Monitor & Event Name & Check Type & Context \\
\hline Student Checks & DoAssignment & PreConditions & Student@ebe5687 \\
Student Checks & DoFinal & PreConditions & Student@ebe5687 \\
Student Checks & DoFinal & PreConditions & Student@ebe5687 \\
Student Checks & DoFinal & PreConditions & Student@ebe5687 \\
\hline
\end{tabular}

\section{B.4 Student Scenario RegisterForCourses Log}

\begin{tabular}{|c|c|c|}
\hline Monitor & Event Name & Context \\
\hline $\begin{array}{l}\text { Student Scenario } \\
\text { RegisterForCourses }\end{array}$ & Trigger & Student@ebe5687 \\
\hline $\begin{array}{l}\text { Student Scenario } \\
\text { RegisterForCourses }\end{array}$ & Trigger & Student@479482ea \\
\hline $\begin{array}{l}\text { Student Scenario } \\
\text { RegisterForCourses }\end{array}$ & Trigger & Student@671ef55c \\
\hline $\begin{array}{l}\text { Student Scenario } \\
\text { RegisterForCourses }\end{array}$ & SelectCourse & Student@ebe5687 \\
\hline $\begin{array}{l}\text { Student Scenario } \\
\text { RegisterForCourses }\end{array}$ & RegisterStudentForCourse & Student@ebe5687 \\
\hline $\begin{array}{l}\text { Student Scenario } \\
\text { RegisterForCourses }\end{array}$ & RegisterCourse & Student@ebe5687 \\
\hline
\end{tabular}




\begin{tabular}{|l|l|l|}
\hline $\begin{array}{l}\text { Student Scenario } \\
\text { RegisterForCourses }\end{array}$ & SelectCourse & Student@ebe5687 \\
\hline $\begin{array}{l}\text { Student Scenario } \\
\text { RegisterForCourses }\end{array}$ & RegisterStudentForCourse & Student@ebe5687 \\
\hline $\begin{array}{l}\text { Student Scenario } \\
\text { RegisterForCourses }\end{array}$ & RegisterCourse & Student@ebe5687 \\
\hline $\begin{array}{l}\text { Student Scenario } \\
\text { RegisterForCourses }\end{array}$ & SelectCourse & Student@ebe5687 \\
\hline $\begin{array}{l}\text { Student Scenario } \\
\text { RegisterForCourses }\end{array}$ & RegisterStudentForCourse & Student@ebe5687 \\
\hline $\begin{array}{l}\text { Student Scenario } \\
\text { RegisterForCourses }\end{array}$ & RegisterCourse & Student@ebe5687 \\
\hline $\begin{array}{l}\text { Student Scenario } \\
\text { RegisterForCourses }\end{array}$ & SelectCourse & Student@ebe5687 \\
\hline $\begin{array}{l}\text { Student Scenario } \\
\text { RegisterForCourses }\end{array}$ & RegisterStudentForCourse \\
\hline $\begin{array}{l}\text { Student Scenario } \\
\text { RegisterForCourses }\end{array}$ & RegisterCourse & Student@ebe5687 \\
\hline Rtudent Scenario & Terminate 5687 \\
\hline
\end{tabular}




\begin{tabular}{|c|c|c|}
\hline $\begin{array}{l}\text { Student Scenario } \\
\text { RegisterForCourses }\end{array}$ & SelectCourse & Student@479482ea \\
\hline $\begin{array}{l}\text { Student Scenario } \\
\text { RegisterForCourses }\end{array}$ & RegisterStudentForCourse & Student@479482ea \\
\hline $\begin{array}{l}\text { Student Scenario } \\
\text { RegisterForCourses }\end{array}$ & RegisterCourse & Student@479482ea \\
\hline $\begin{array}{l}\text { Student Scenario } \\
\text { RegisterForCourses }\end{array}$ & SelectCourse & Student@479482ea \\
\hline $\begin{array}{l}\text { Student Scenario } \\
\text { RegisterForCourses }\end{array}$ & RegisterStudentForCourse & Student@479482ea \\
\hline $\begin{array}{l}\text { Student Scenario } \\
\text { RegisterForCourses }\end{array}$ & RegisterCourse & Student@479482ea \\
\hline $\begin{array}{l}\text { Student Scenario } \\
\text { RegisterForCourses }\end{array}$ & Terminate & Student@479482ea \\
\hline $\begin{array}{l}\text { Student Scenario } \\
\text { RegisterForCourses }\end{array}$ & SelectCourse & Student@671ef55c \\
\hline $\begin{array}{l}\text { Student Scenario } \\
\text { RegisterForCourses }\end{array}$ & RegisterStudentForCourse & Student@671ef55c \\
\hline $\begin{array}{l}\text { Student Scenario } \\
\text { RegisterForCourses }\end{array}$ & RegisterCourse & Student@671ef55c \\
\hline
\end{tabular}




\section{B.5 Student Scenario TakeCourses Log}

\begin{tabular}{|c|c|c|c|}
\hline Monitor & & Event Name & Context \\
\hline $\begin{array}{l}\text { Student Scenario } \\
\text { Courses Parent }\end{array}$ & Take- & Trigger & Student@ebe5687 \\
\hline $\begin{array}{l}\text { Student Scenario } \\
\text { Courses Sub }\end{array}$ & Take- & Trigger & $\begin{array}{l}\text { Student@ebe5687; } \\
\text { Course@1720d359 }\end{array}$ \\
\hline $\begin{array}{l}\text { Student Scenario } \\
\text { Courses Sub }\end{array}$ & Take- & Trigger & $\begin{array}{l}\text { Student@ebe5687; } \\
\text { Course@51cf1d17 }\end{array}$ \\
\hline $\begin{array}{l}\text { Student Scenario } \\
\text { Courses Sub }\end{array}$ & Take- & Trigger & $\begin{array}{l}\text { Student@ebe5687; } \\
\text { Course@3c8e683a }\end{array}$ \\
\hline $\begin{array}{l}\text { Student Scenario } \\
\text { Courses Sub }\end{array}$ & Take- & Trigger & $\begin{array}{l}\text { Student@ebe5687; } \\
\text { Course@295cd6e5 }\end{array}$ \\
\hline $\begin{array}{l}\text { Student Scenario } \\
\text { Courses Parent }\end{array}$ & Take- & Trigger & Student@479482ea \\
\hline $\begin{array}{l}\text { Student Scenario } \\
\text { Courses Sub }\end{array}$ & Take- & Trigger & $\begin{array}{l}\text { Student@479482ea; } \\
\text { Course@51cf1d17 }\end{array}$ \\
\hline $\begin{array}{l}\text { Student Scenario } \\
\text { Courses Sub }\end{array}$ & Take- & Trigger & $\begin{array}{l}\text { Student@479482ea; } \\
\text { Course@295cd6e5 }\end{array}$ \\
\hline $\begin{array}{l}\text { Student Scenario } \\
\text { Courses Parent }\end{array}$ & Take- & Trigger & Student@671ef55c \\
\hline
\end{tabular}




\begin{tabular}{|c|c|c|c|}
\hline $\begin{array}{l}\text { Student Scenario } \\
\text { Courses Sub }\end{array}$ & Take- & Trigger & $\begin{array}{l}\text { Student@671ef55c; } \\
\text { Course@e20699a }\end{array}$ \\
\hline $\begin{array}{l}\text { Student Scenario } \\
\text { Courses Sub }\end{array}$ & Take- & DoAssignment & $\begin{array}{l}\text { Student@479482ea; } \\
\text { Course@51cf1d17 }\end{array}$ \\
\hline $\begin{array}{l}\text { Student Scenario } \\
\text { Courses Sub }\end{array}$ & Take- & DoAssignment & $\begin{array}{l}\text { Student@ebe5687; } \\
\text { Course@1720d359 }\end{array}$ \\
\hline $\begin{array}{l}\text { Student Scenario } \\
\text { Courses Sub }\end{array}$ & Take- & DoAssignment & $\begin{array}{l}\text { Student@ebe5687; } \\
\text { Course@51cf1d17 }\end{array}$ \\
\hline $\begin{array}{l}\text { Student Scenario } \\
\text { Courses Sub }\end{array}$ & Take- & DoMidterm & $\begin{array}{l}\text { Student@ebe5687; } \\
\text { Course@51cf1d17 }\end{array}$ \\
\hline $\begin{array}{l}\text { Student Scenario } \\
\text { Courses Sub }\end{array}$ & Take- & DoAssignment & $\begin{array}{l}\text { Student@ebe5687; } \\
\text { Course@51cf1d17 }\end{array}$ \\
\hline $\begin{array}{l}\text { Student Scenario } \\
\text { Courses Sub }\end{array}$ & Take- & DoMidterm & $\begin{array}{l}\text { Student@ebe5687; } \\
\text { Course@1720d359 }\end{array}$ \\
\hline $\begin{array}{l}\text { Student Scenario } \\
\text { Courses Sub }\end{array}$ & Take- & DoAssignment & $\begin{array}{l}\text { Student@ebe5687; } \\
\text { Course@1720d359 }\end{array}$ \\
\hline $\begin{array}{l}\text { Student Scenario } \\
\text { Courses Sub }\end{array}$ & Take- & DoMidterm & $\begin{array}{l}\text { Student@ebe5687; } \\
\text { Course@295cd6e5 }\end{array}$ \\
\hline $\begin{array}{l}\text { Student Scenario } \\
\text { Courses Sub }\end{array}$ & Take- & DoAssignment & $\begin{array}{l}\text { Student@ebe5687; } \\
\text { Course@295cd6e5 }\end{array}$ \\
\hline
\end{tabular}




\begin{tabular}{|c|c|c|c|}
\hline $\begin{array}{l}\text { Student Scenario } \\
\text { Courses Sub }\end{array}$ & Take- & DoAssignment & $\begin{array}{l}\text { Student@ebe5687; } \\
\text { Course@3c8e683a }\end{array}$ \\
\hline $\begin{array}{l}\text { Student Scenario } \\
\text { Courses Sub }\end{array}$ & Take- & DoMidterm & $\begin{array}{l}\text { Student@ebe5687; } \\
\text { Course@3c8e683a }\end{array}$ \\
\hline $\begin{array}{l}\text { Student Scenario } \\
\text { Courses Sub }\end{array}$ & Take- & DoAssignment & $\begin{array}{l}\text { Student@ebe5687; } \\
\text { Course@1720d359 }\end{array}$ \\
\hline $\begin{array}{l}\text { Student Scenario } \\
\text { Courses Sub }\end{array}$ & Take- & DoAssignment & $\begin{array}{l}\text { Student@ebe5687; } \\
\text { Course@3c8e683a }\end{array}$ \\
\hline $\begin{array}{l}\text { Student Scenario } \\
\text { Courses Sub }\end{array}$ & Take- & DoMidterm & $\begin{array}{l}\text { Student@ebe5687; } \\
\text { Course@295cd6e5 }\end{array}$ \\
\hline $\begin{array}{l}\text { Student Scenario } \\
\text { Courses Sub }\end{array}$ & Take- & DoAssignment & $\begin{array}{l}\text { Student@ebe5687; } \\
\text { Course@3c8e683a }\end{array}$ \\
\hline $\begin{array}{l}\text { Student Scenario } \\
\text { Courses Sub }\end{array}$ & Take- & DoMidterm & $\begin{array}{l}\text { Student@ebe5687; } \\
\text { Course@1720d359 }\end{array}$ \\
\hline $\begin{array}{l}\text { Student Scenario } \\
\text { Courses Sub }\end{array}$ & Take- & DoAssignment & $\begin{array}{l}\text { Student@ebe5687; } \\
\text { Course@3c8e683a }\end{array}$ \\
\hline $\begin{array}{l}\text { Student Scenario } \\
\text { Courses Sub }\end{array}$ & Take- & CourseComplete & $\begin{array}{l}\text { Student@ebe5687; } \\
\text { Course@3c8e683a }\end{array}$ \\
\hline $\begin{array}{l}\text { Student Scenario } \\
\text { Courses Parent }\end{array}$ & Take- & CourseComplete & Student@ebe5687 \\
\hline
\end{tabular}




\begin{tabular}{|c|c|c|c|}
\hline $\begin{array}{l}\text { Student Scenario } \\
\text { Courses Sub }\end{array}$ & Take- & DoFinal & $\begin{array}{l}\text { Student@ebe5687; } \\
\text { Course@1720d359 }\end{array}$ \\
\hline $\begin{array}{l}\text { Student Scenario } \\
\text { Courses Sub }\end{array}$ & Take- & CourseComplete & $\begin{array}{l}\text { Student@ebe5687; } \\
\text { Course@1720d359 }\end{array}$ \\
\hline $\begin{array}{l}\text { Student Scenario } \\
\text { Courses Parent }\end{array}$ & Take- & CourseComplete & Student@ebe5687 \\
\hline $\begin{array}{l}\text { Student Scenario } \\
\text { Courses Sub }\end{array}$ & Take- & DoFinal & $\begin{array}{l}\text { Student@ebe5687; } \\
\text { Course@51cf1d17 }\end{array}$ \\
\hline $\begin{array}{l}\text { Student Scenario } \\
\text { Courses Sub }\end{array}$ & Take- & CourseComplete & $\begin{array}{l}\text { Student@ebe5687; } \\
\text { Course@51cf1d17 }\end{array}$ \\
\hline $\begin{array}{l}\text { Student Scenario } \\
\text { Courses Parent }\end{array}$ & Take- & CourseComplete & Student@ebe5687 \\
\hline $\begin{array}{l}\text { Student Scenario } \\
\text { Courses Sub }\end{array}$ & Take- & DoFinal & $\begin{array}{l}\text { Student@ebe5687; } \\
\text { Course@295cd6e5 }\end{array}$ \\
\hline $\begin{array}{l}\text { Student Scenario } \\
\text { Courses Sub }\end{array}$ & Take- & CourseComplete & $\begin{array}{l}\text { Student@ebe5687; } \\
\text { Course@295cd6e5 }\end{array}$ \\
\hline $\begin{array}{l}\text { Student Scenario } \\
\text { Courses Parent }\end{array}$ & Take- & CourseComplete & Student@ebe5687 \\
\hline $\begin{array}{l}\text { Student Scenario } \\
\text { Courses Parent }\end{array}$ & Take- & Terminate & Student@ebe5687 \\
\hline
\end{tabular}




\section{B.6 University Checks Log}

\begin{tabular}{|l|l|l|l|}
\hline Monitor & Event Name & Check Type & Context \\
\hline University Checks & newInstance & null & University@1699860f \\
University Checks & RegisterStudentForCourse & Postconditions & University@1699860f \\
University Checks & RegisterStudentForCourse & Postconditions & University@1699860f \\
University Checks & RegisterStudentForCourse & Postconditions & University@1699860f \\
University Checks & RegisterStudentForCourse & Postconditions & University@1699860f \\
University Checks & RegisterStudentForCourse & Postconditions & University@1699860f \\
University Checks & RegisterStudentForCourse & Postconditions & University@1699860f \\
University Checks & RegisterStudentForCourse & Postconditions & University@1699860f \\
University Checks & ReportMark & Postconditions & University@1699860f \\
University Checks & ReportMark & Postconditions & University@1699860f \\
University Checks & ReportMark & Postconditions & University@1699860f \\
University Checks & ReportMark & Postconditions & University@1699860f \\
University Checks & ReportMark & Prostconditions & University@1699860f \\
University Checks & ReportMark & University@1699860f \\
University Checks & ReportMark & Postconditions & University@1699860f \\
University Checks & DestroyCourse & University@1699860f \\
\hline
\end{tabular}




\begin{tabular}{|l|l|l|l|}
\hline Monitor & Event Name & Check Type & Context \\
\hline University Checks & DestroyCourse & Preconditions & University@1699860f \\
University Checks & DestroyCourse & Postconditions & University@1699860f \\
University Checks & DestroyCourse & Preconditions & University@1699860f \\
University Checks & DestroyCourse & Postconditions & University@1699860f \\
University Checks & DestroyCourse & Preconditions & University@1699860f \\
University Checks & DestroyCourse & Postconditions & University@1699860f \\
University Checks & DestroyCourse & Preconditions & University@1699860f \\
University Checks & DestroyCourse & Postconditions & University@1699860f \\
\hline
\end{tabular}

\section{B.7 University Scenario CreateCourses Log}

\begin{tabular}{|l|l|l|}
\hline Monitor & Event Name & Context \\
\hline University Scenario CreateCourses & Trigger & University@1699860f \\
University Scenario CreateCourses & CreateCourse & University@1699860f \\
University Scenario CreateCourses & CreateCourse & University@1699860f \\
University Scenario CreateCourses & CreateCourse & University@1699860f \\
University Scenario CreateCourses & CreateCourse & University@1699860f \\
University Scenario CreateCourses & CreateCourse & University@1699860f \\
University Scenario CreateCourses & Terminate & University@1699860f \\
\hline
\end{tabular}




\section{B.8 University Scenario CreateStudents Log}

\begin{tabular}{|l|l|l|}
\hline Monitor & Event Name & Context \\
\hline University Scenario CreateStudents & Trigger & University@1699860f \\
University Scenario CreateStudents & CreateStudent & University@1699860f \\
University Scenario CreateStudents & CreateStudent & University@1699860f \\
University Scenario CreateStudents & CreateStudent & University@1699860f \\
\hline
\end{tabular}

\section{B.9 University Scenario Term Log}

\begin{tabular}{|l|l|l|}
\hline Monitor & Event Name & Context \\
\hline University Term Scenario & Trigger & University@57142653 \\
University Term Scenario & CreateCourse & University@57142653 \\
University Term Scenario & CreateCourse & University@57142653 \\
University Term Scenario & CreateCourse & University@57142653 \\
University Term Scenario & CreateCourse & University@57142653 \\
University Term Scenario & CreateCourse & University@57142653 \\
University Term Scenario & TermStarted & University@57142653 \\
University Term Scenario & LastDayToDrop & University@57142653 \\
University Term Scenario & TermEnded & University@57142653 \\
\hline
\end{tabular}




\begin{tabular}{|l|l|l|}
\hline Monitor & Event Name & Context \\
\hline University Term Scenario & MarksRecorded & University@57142653 \\
University Term Scenario & MarksRecorded & University@57142653 \\
University Term Scenario & MarksRecorded & University@57142653 \\
University Term Scenario & MarksRecorded & University@57142653 \\
University Term Scenario & MarksRecorded & University@57142653 \\
University Term Scenario & CalculatePassFail & University@57142653 \\
University Term Scenario & CalculatePassFail & University@57142653 \\
University Term Scenario & CalculatePassFail & University@57142653 \\
University Term Scenario & DestroyCourse & University@57142653 \\
University Term Scenario & DestroyCourse & University@57142653 \\
University Term Scenario & DestroyCourse & University@57142653 \\
University Term Scenario & DestroyCourse & University@57142653 \\
University Term Scenario & DestroyCourse & University@57142653 \\
\hline
\end{tabular}

\section{B.10 Test Driver Program}

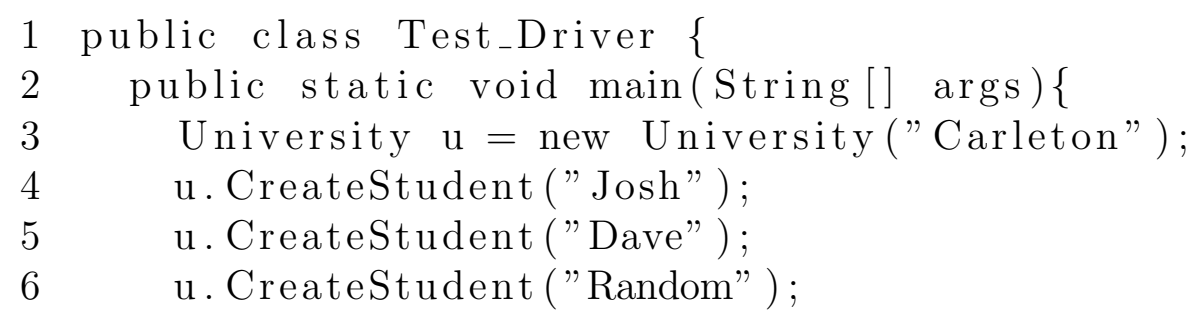




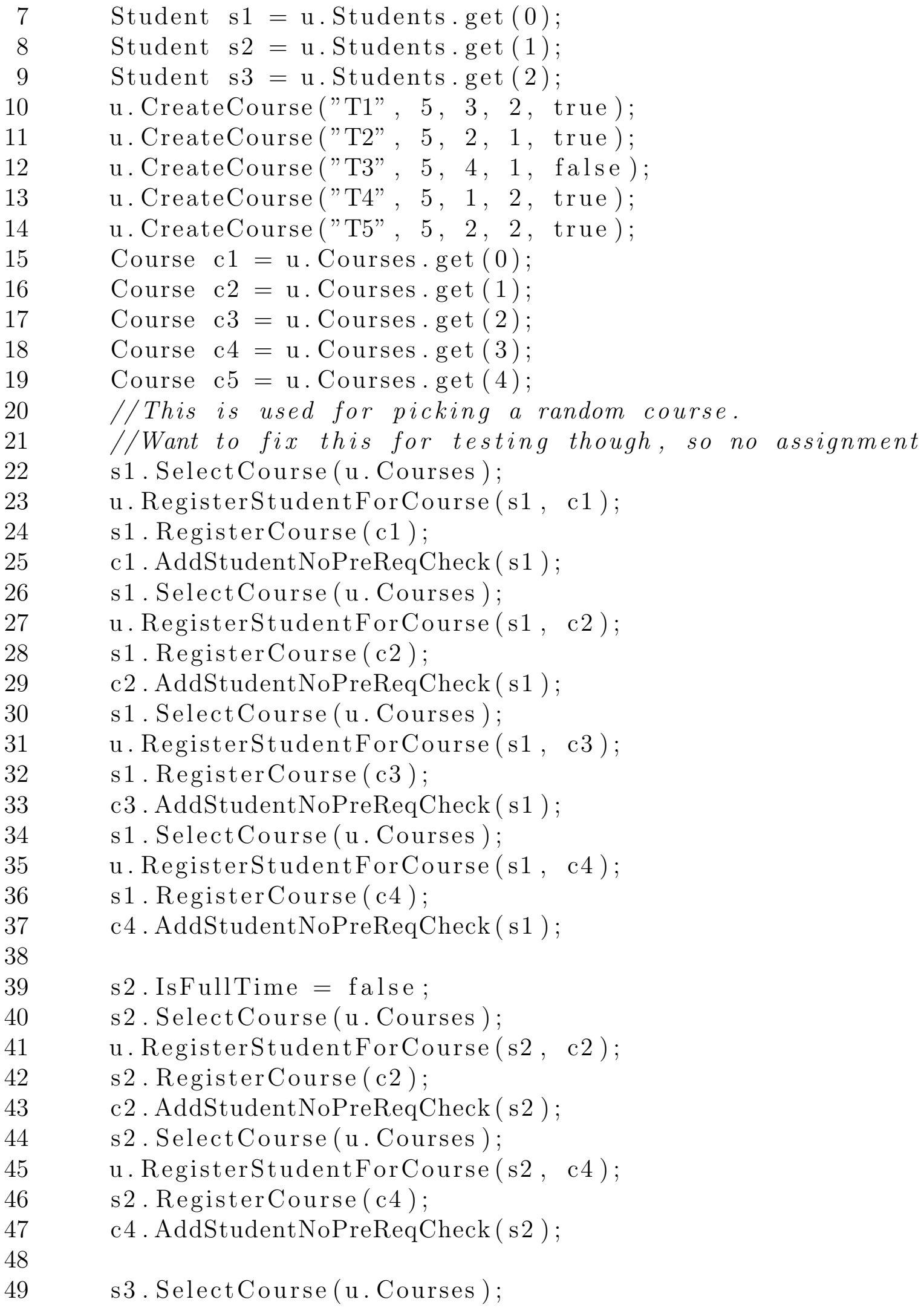




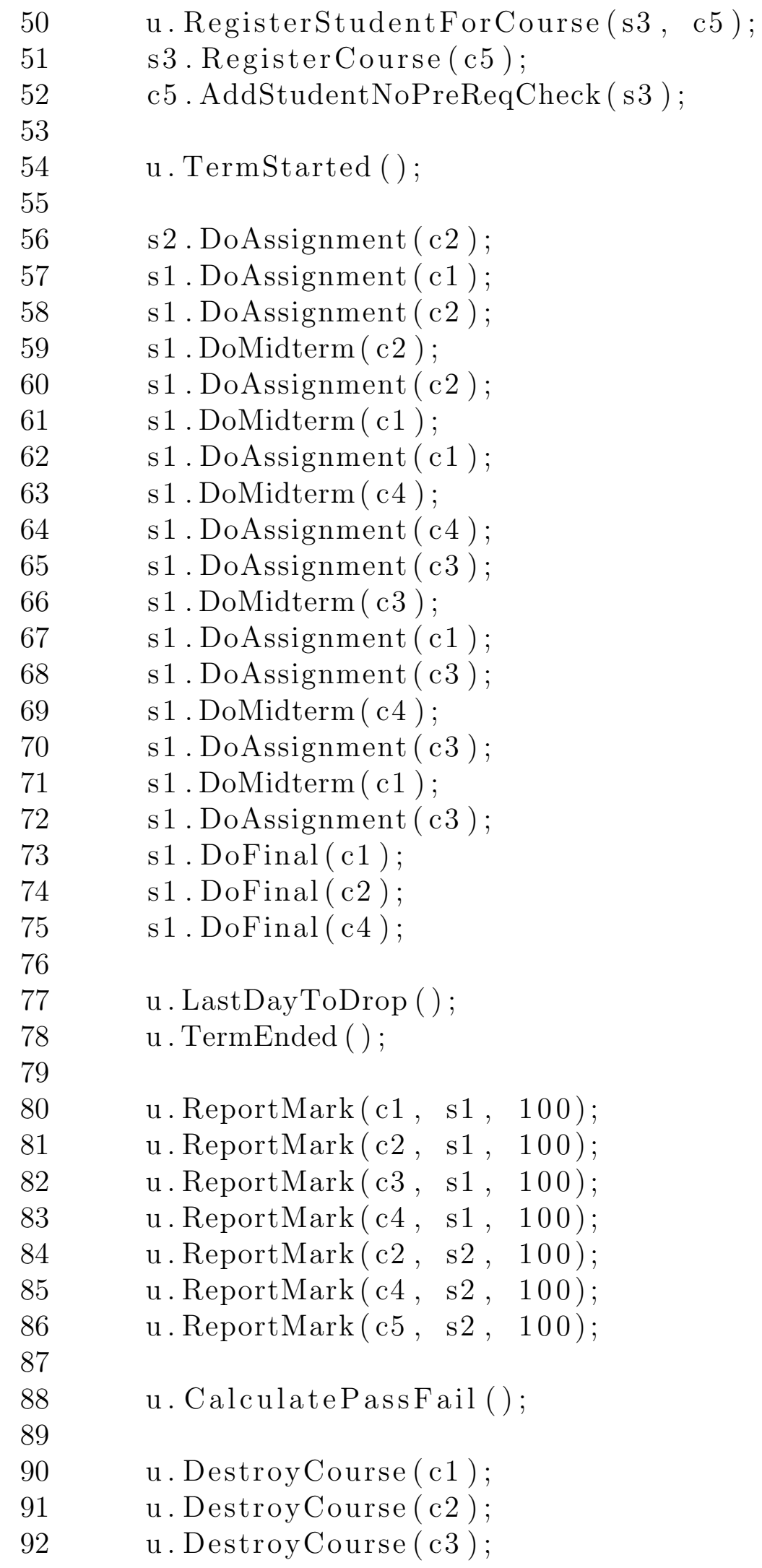


93 u. DestroyCourse (c4);

94 u. DestroyCourse ( c 5);

$95\}$

$96\}$ 